\title{
THE ALLWISE MOTION SURVEY AND THE QUEST FOR COLD SUBDWARFS
}

\author{
J. Davy Kirkpatrick ${ }^{1}$, Adam Schneider ${ }^{2}$, Sergio Fajardo-Acosta ${ }^{1}$, Christopher R. Gelino ${ }^{1,3}$, Gregory N. Mace ${ }^{4}$, \\ Edward L. Wright ${ }^{4}$, Sarah E. Logsdon ${ }^{4}$, Ian S. McLean ${ }^{4}$, Michael C. Cushing $^{2}$, Michael F. Skrutskie $^{5}$, \\ Peter R. Eisenhardt ${ }^{6}$, Daniel Stern ${ }^{6}$, Mislav Baloković ${ }^{7}$, Adam J. Burgasser ${ }^{8}$, Jacqueline K. Faherty ${ }^{9}$, \\ George B. Lansbury ${ }^{10}$, J. A. Rich ${ }^{11}$, Nathalie SkrzypeK ${ }^{12}$, John W. Fowler ${ }^{1}$, Roc M. Cutri ${ }^{1}$, Frank J. Masci ${ }^{1}$, \\ Tim Conrow ${ }^{1}$, Carl J. Grillmair ${ }^{1}$, Howard L. McCallon ${ }^{1}$, Charles A. Beichman $^{1,3}$, and Kenneth A. Marsh ${ }^{13}$ \\ ${ }^{1}$ Infrared Processing and Analysis Center, MS 100-22, California Institute of Technology, Pasadena, CA 91125, USA; davy@ipac.caltech.edu \\ ${ }^{2}$ Department of Physics and Astronomy, MS 111, University of Toledo, 2801 West Bancroft St., Toledo, OH 43606-3328, USA \\ ${ }^{3}$ NASA Exoplanet Science Institute, MS 100-22, California Institute of Technology, Pasadena, CA 91125, USA \\ ${ }^{4}$ Department of Physics and Astronomy, UCLA, 430 Portola Plaza, Box 951547, Los Angeles, CA 90095-1547, USA \\ ${ }^{5}$ Department of Astronomy, University of Virginia, Charlottesville, VA 22904, USA \\ ${ }^{6}$ NASA Jet Propulsion Laboratory, 4800 Oak Grove Drive, Pasadena, CA 91109, USA \\ ${ }^{7}$ California Institute of Technology, MC 249-17, Pasadena, CA 91125, USA \\ ${ }^{8}$ Department of Physics, University of California, San Diego, CA 92093, USA \\ ${ }^{9}$ Department of Terrestrial Magnetism, Carnegie Institution of Washington, Washington, DC 20015, USA \\ ${ }^{10}$ Department of Physics, Durham University, Durham DH1 3LE, UK \\ ${ }^{11}$ Observatories of the Carnegie Institution of Washington, 813 Santa Barbara Street, Pasadena, CA 91101, USA \\ 12 Astro Group, Imperial College London, Blackett Laboratory, Prince Consort Road, London SW7 2AZ, UK \\ ${ }^{13}$ School of Physics and Astronomy, Cardiff University, Cardiff CF24 3AA, UK \\ Received 2014 January 10; accepted 2014 February 3; published 2014 February 24
}

\begin{abstract}
The AllWISE processing pipeline has measured motions for all objects detected on Wide-field Infrared Survey Explorer (WISE) images taken between 2010 January and 2011 February. In this paper, we discuss new capabilities made to the software pipeline in order to make motion measurements possible, and we characterize the resulting data products for use by future researchers. Using a stringent set of selection criteria, we find 22,445 objects that have significant AllWISE motions, of which 3525 have motions that can be independently confirmed from earlier Two Micron All Sky Survey (2MASS) images, yet lack any published motions in SIMBAD. Another 58 sources lack 2MASS counterparts and are presented as motion candidates only. Limited spectroscopic follow-up of this list has already revealed eight new L subdwarfs. These may provide the first hints of a "subdwarf gap" at mid-L types that would indicate the break between the stellar and substellar populations at low metallicities (i.e., old ages). Another object in the motion list-WISEA J154045.67-510139.3 - is a bright ( $J \approx 9 \mathrm{mag}$ ) object of type M6; both the spectrophotometric distance and a crude preliminary parallax place it $\sim 6$ pc from the Sun. We also compare our list of motion objects to the recently published list of 762 WISE motion objects from Luhman. While these first large motion studies with WISE data have been very successful in revealing previously overlooked nearby dwarfs, both studies missed objects that the other found, demonstrating that many other nearby objects likely await discovery in the AllWISE data products.
\end{abstract}

Key words: brown dwarfs - catalogs - solar neighborhood - stars: fundamental parameters - stars: low-mass subdwarfs

Online-only material: color figures, machine-readable table

\section{INTRODUCTION}

The Wide-field Infrared Survey Explorer (WISE; Wright et al. 2010) was built to survey the sky simultaneously in four bands with central wavelengths of 3.4, 4.6, 12, and $22 \mu \mathrm{m}$ (hereafter referred to as $W 1, W 2, W 3$, and $W 4$, respectively). The fourband cryogenic mission covered the sky 1.2 times between 2010 January 07 and 2010 August 06. After the solid hydrogen cryogen was depleted in the outer tank, data covering $30 \%$ of the sky continued to be acquired in the three-band ( $W 1, W 2$, and W3) cryogenic mission until 2010 September 29, when cryogen in the inner tank was exhausted. WISE surveyed $70 \%$ of the sky in the two-band ( $W 1$ and $W 2$ ) post-cryogenic NEOWISE mission (Mainzer et al. 2011) until the satellite was placed into hibernation on 2011 February 01. In total, WISE surveyed the full sky twice in at least two bands and $20 \%$ of the sky a third time, with each coverage epoch separated by approximately six months.
Data were processed and released separately for the cryogenic and post-cryogenic missions. The aim of the AllWISE program was to combine all of the data from the WISE mission to leverage the full depth and time history of the $W 1$ and $W 2$ data. Specifically, one of these goals was to use the full WISE data to perform the first all-sky proper motion survey at these wavelengths. In addition to the exciting prospects created by doing a kinematic survey in a new wavelength regime, AllWISE processing-because of the short, six-month time baseline between epochs - enables us to search for very high motion objects that may have been overlooked by the longer time baselines of earlier all-sky motion surveys. In these surveys, an object could be "lost" between consecutive views. A case in point is the first motion discovery from AllWISE, the usdM3 star WISEA J070720.50+170532.7 (Wright et al. 2013), which is moving at $1^{\prime \prime} .8 \mathrm{yr}^{-1}$ and was missed by all previous motion surveys despite being in the more heavily studied northern hemisphere, being easily detected at optical 
wavelengths, and falling in an uncomplicated region free of source confusion.

In this paper, we describe how motions are measured in AllWISE (Section 2), characterize the resulting motions and present caveats to users of the AllWISE data products (Section 3), present a catalog of new motion discoveries (Section 4), compare that to the recent list of WISE motion discoveries published by Luhman (2014; Section 5), discuss our spectroscopic follow-up of some of the discoveries (Section 6), and highlight a couple of specific science cases-the hunt for rare, L-type subdwarfs (Section 7) and the search for missing members of the immediate solar neighborhood (Section 8) - that we have already explored using the discovery list. In Section 9, we provide a summary of our AllWISE results thus far.

\section{MEASURING MOTIONS IN ALLWISE}

AllWISE combines all previous WISE single-exposure images (called Level $1 \mathrm{~b}$ frames) that satisfy a minimum quality standard. These individual frames are match-filtered and coadded on pre-defined $1.56 \times 1.56$ Atlas Tile footprints on the sky. Pixel outlier rejection is used to suppress transient features and objects of extremely high motion (e.g., asteroids and satellite streaks).

In processing of the earlier WISE data sets, the following procedure was used for source detection and astrometric/ photometric measurement. A list of detections from a multiband signal-to-noise ratio map of the coadded images was first generated for each Atlas Tile. At the position of each detection in the list, a point response function fit was then performed to measure the source position and flux via a $\chi^{2}$ minimization procedure on the stack of individual frames that generated the coadd. Because the WISE beam size is large (the fullwidth at half-maximum, FWHM, is $12^{\prime \prime}$ at $W 4$ ), the deblending of overlapping detections - and the further deconvolution of those detections into possible multiple components-is handled through a $\chi^{2}$ minimization procedure explained in more detail in the WISE All-Sky Release Explanatory Supplement. ${ }^{14}$

During AllWISE processing, the source position measurement model was augmented with linear motion terms in R.A. and Decl. (the "motion fit"). In AllWISE processing, the fit excluding these new motion terms (the "stationary fit") is performed first, and the full set of photometric parameters is computed. The motion fit begins with the stationary position as the initial position estimate at a fiducial time that is the flux-weighted mean observational epoch of each source. Because of the effects of noise on the non-linear $\chi^{2}$ minimization algorithm, it is sensitive to the initial estimates used to start the minimization search. Of the several ways to weigh the epoch averaging, flux weighting based on the stationary-fit single-frame flux solutions was found to be the most effective in reproducing motions of known moving objects. Readers are encouraged to consult the AllWISE Release Explanatory Supplement ${ }^{15}$ for additional details.

As a result of this addition to the AllWISE processing, motions are now measured for all coadd-detected WISE sources, giving researchers a powerful new tool with which to study and/or identify nearby stars and brown dwarfs. This paper characterizes these new motion measurements, discusses the caveats, and showcases two research areas-the search for lowmetallicity stars and brown dwarfs and the hunt for missing

\footnotetext{
14 See http://wise2.ipac.caltech.edu/docs/release/allsky/expsup/.

15 See http://wise2.ipac.caltech.edu/docs/release/allwise/expsup/.
}
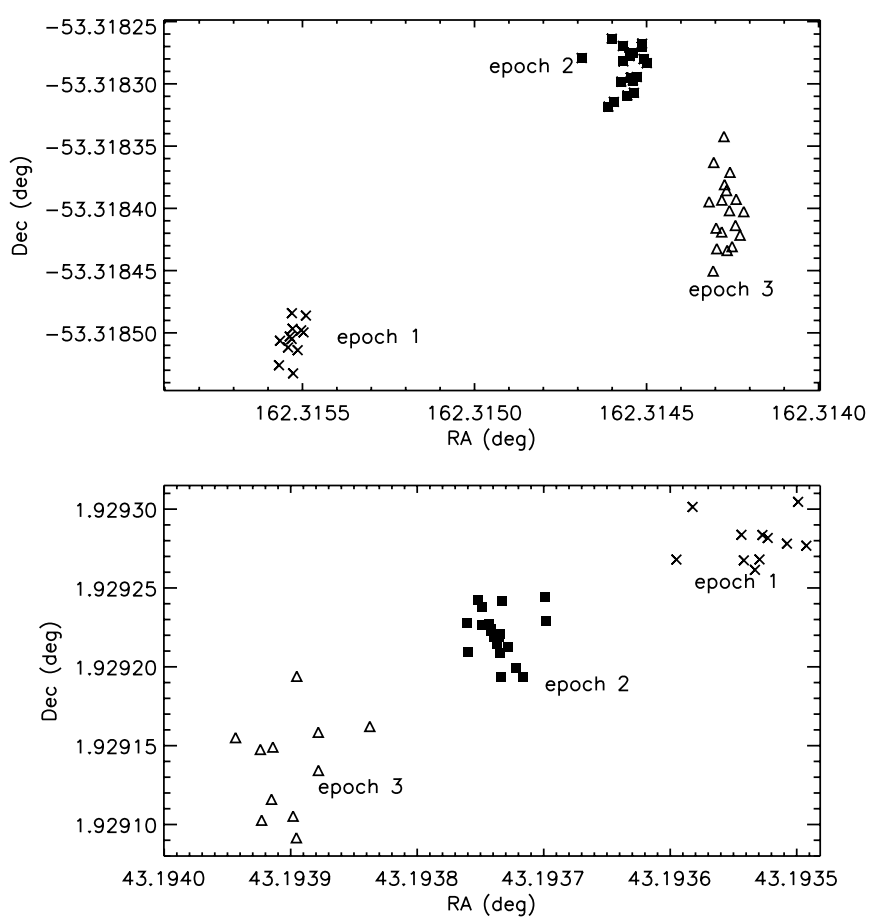

Figure 1. Astrometry measured from the individual Level $1 \mathrm{~b}$ frames for the nearby L+T dwarf binary WISE J104915.57-531906.1 (top) and LHS 161 (bottom). The three clusters of points labeled "epoch 1," "epoch 2," and "epoch 3" show the WISE data separated by six-month intervals.

members of the solar neighborhood-that are particularly well suited for this data set.

\section{ANALYSIS OF THE ALLWISE MOTION PRODUCTS}

\subsection{Apparent Motion versus Proper Motion}

The AllWISE time baseline is typically between six months and a year for any point on the sky. Except for ecliptic polar regions, the time sampling will be confined to discrete epochs separated by roughly six months. Multiple measurements, spanning the range of a few days, are taken at each epoch. Most of the sky has two epochs covering six months, and $\sim 20 \%$ of the sky has three epochs spanning a time range of one year.

It is important to understand what this time sampling means for motion measurements. In general, it is the closest objects to the Sun that exhibit the largest proper motions. Being close, these objects will also exhibit substantial parallax. Because data are taken near $90^{\circ}$ solar elongation, WISE observed these objects at their maximum parallax factors. As an example, an object seen at two WISE epochs will have motion that is a combination of half its yearly proper motion (because the two epochs are only separated by six months) and twice its parallax (because the vantage point between epochs has shifted by 2 AU). Having only two epochs, AllWISE cannot disentangle the effects of parallax and proper motion and thus measures only apparent motion on the sky as opposed to proper motion.

Figure 1 (top panel) shows the individual Level 1b frame measurements of the position of the nearby L+T dwarf binary WISE J104915.57-531906.1. This source has three epochs of coverage in AllWISE. The motion is not linear because this binary's parallax $(\pi=0$ '.50) and proper motion $(\mu=$ $2^{\prime \prime} .78 \mathrm{yr}^{-1}$ ) are comparable in size (Luhman 2013). It would be possible to measure the proper motion by using only the epoch 1 and epoch 3 points, since these clusters of points were 


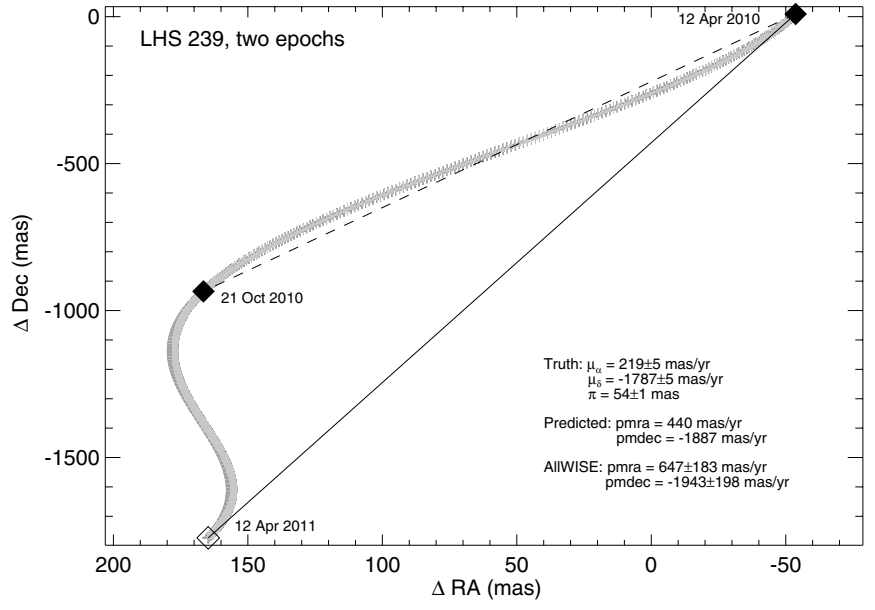

Figure 2. Predicted motion track (gray plus signs, one symbol per day) for the DC12 white dwarf LHS 239. Solid diamonds show the dates of the WISE observations. The open diamond shows the date 12 months after the initial WISE observation. AllWISE measures a motion over six months as noted by the dashed line (that is, the reported yearly motion will be twice this long), whereas the true proper motion over a full year is denoted by the solid line.

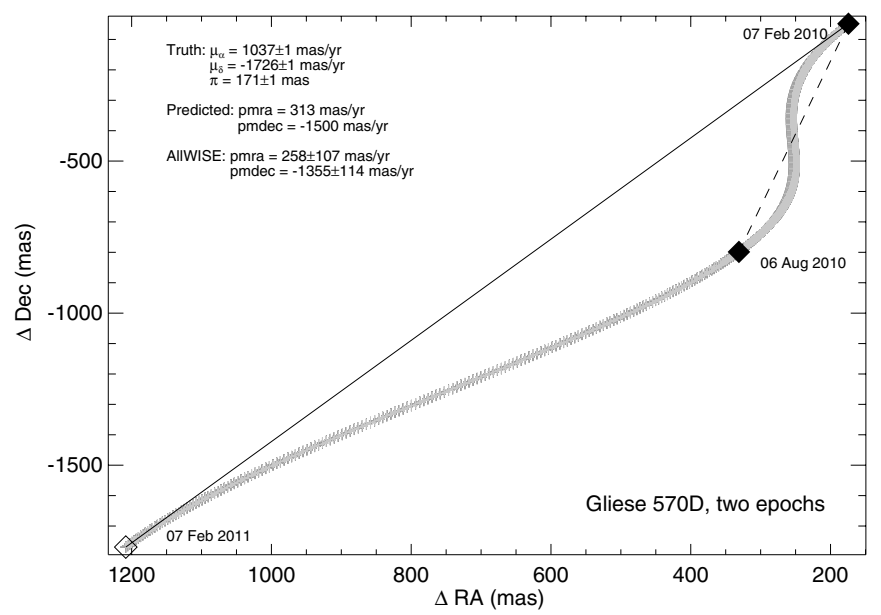

Figure 3. Predicted motion track (gray plus signs, one symbol per day) for the T7.5 brown dwarf Gliese 570D. Solid diamonds show the dates of the WISE observations. The open diamond shows the date 12 months after the initial WISE observation. AllWISE measures a motion over six months as noted by the dashed line, whereas the true proper motion over a full year is denoted by the solid line.

taken at the same parallax factor; however, AllWISE uses data from all three epochs and thus will measure a motion that is not the actual "proper" motion. The extreme M subdwarf LHS 161 in Figure 1 (bottom panel) demonstrates a case at the opposite extreme, where the parallax is swamped by a much larger proper motion ( $\pi=0^{\prime}$.025 and $\mu=1$.. $455 \mathrm{yr}^{-1}$; van Altena et al. 1995). As expected for this case, the three separate epochs of data more closely follow a linear progression in time.

This can be further demonstrated with a couple of examples drawn from areas with two-epoch coverage, which represents the majority of WISE data. Figure 2 shows the predicted path of the white dwarf LHS 239 on the sky, and Figure 3 shows the path for the T dwarf Gliese $570 \mathrm{D}$. In each of these plots, the gray track shows the predicted path of the object from the date of the first WISE epoch to a date one year later. The date of the second (i.e., final) WISE epoch, approximately six months after the first, is also noted. The motion that AllWISE measures is the positional difference between the start of the track and the point six months

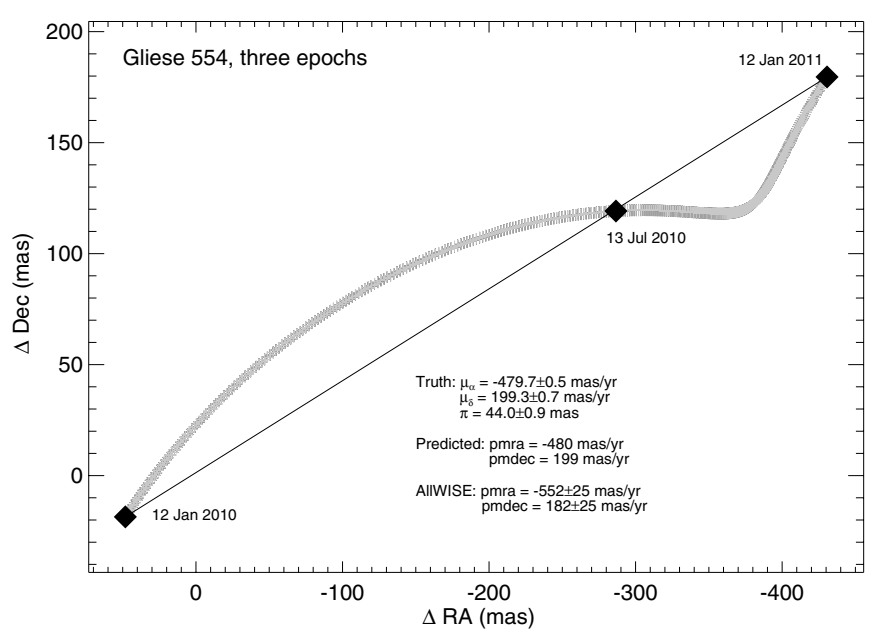

Figure 4. Predicted motion track (gray plus signs, one symbol per day) for the K3 dwarf Gliese 554. Dates of the WISE data points (denoted by solid diamonds) cover a full year. AllWISE measures a motion that is very close to the true proper motion in this case.

later, whereas the true proper motion would be that measured between the start of the track and the point twelve months later. The true proper motion and parallax, the predicted motion from AllWISE, and the actual AllWISE values are given in the legend of both figures. Note that the actual AllWISE measurements are, within their errors, identical to the predicted values, although not necessarily close to the true proper motion measures. As stated earlier, these AllWISE measurements should be regarded as apparent motion values (parallax + proper motion) and not as pure proper motion values.

Figure 4 illustrates the predicted sky path for the $\mathrm{K}$ dwarf Gliese 554, located in an area with three epochs of coverage. In this case, the WISE data fall almost perfectly along the proper motion vector. It would be expected that the AllWISE motion measure would be very close to the true proper motion value, which is indeed the case. It should be noted that a measurement using only epoch $1+2$ data would have a larger motion than a measurement using only epoch $2+3$ data, although both would have the correct position angle.

Because the AllWISE-measured motions do not decouple proper motion and parallax, these motions cannot be used to predict source positions at other epochs. Rather, their utility is in identifying potentially interesting, nearby objects for further follow-up.

\subsection{Motion Limits}

\subsubsection{What is the Largest Motion Measurable?}

AllWISE is able to identify objects with very high motion. Table 1 lists the 11 highest proper motion systems known

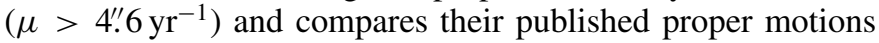
to the motions derived by AllWISE. Most of these sources are extremely bright and heavily saturated on the WISE detectors. Agreement between published values and those in AllWISE is not expected due both to saturation effects and to parallax. Nonetheless, the comparison shows that the highest motion stars can be identified as such in AllWISE data.

Objects with motions as high as Barnard's Star $\left(10.4 \mathrm{yr}^{-1}\right)$ can be successfully recovered with AllWISE, but what is the theoretical limit to the largest motion that AllWISE can measure for a real, astrophysical source? There are sources in the combination of the AllWISE Source Catalog and Reject 
Table 1

Motion Comparisons for the Eleven Highest Proper Motion Systems Known

\begin{tabular}{|c|c|c|c|c|c|c|c|c|c|}
\hline Object Name & Sp. Type & $\begin{array}{c}\text { AllWISE } \\
\text { Designation } \\
\text { (3) }\end{array}$ & $\begin{array}{c}\text { AllWISE } \\
W 1 \\
(\mathrm{mag}) \\
(4)\end{array}$ & $\begin{array}{c}\text { AllWISE } \\
\text { R.A. Motion } \\
\left(\mathrm{mas} \mathrm{yr}^{-1}\right) \\
(5)\end{array}$ & $\begin{array}{c}\text { AllWISE } \\
\text { Decl. Motion } \\
\left(\mathrm{mas} \mathrm{yr}^{-1}\right) \\
(6)\end{array}$ & $\begin{array}{c}\text { Published } \mu_{\alpha} \\
\left(\text { mas yr }^{-1} \text { ) }\right. \\
\text { (7) }\end{array}$ & 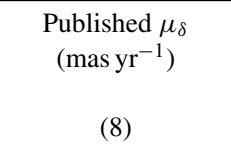 & $\begin{array}{l}\text { Published } \pi \\
\text { (mas) } \\
\text { (9) }\end{array}$ & (10) \\
\hline Barnard's Star & M5 V & WISEA J175747.94+044323.8 & 5.02 & $-1630 \pm 71$ & $13703 \pm 65$ & $-798.58 \pm 1.72$ & $10328.12 \pm 1.22$ & $548.31 \pm 1.51$ & 1,9 \\
\hline Kapteyn's Star & sdM1p & WISEA J051146.81-450204.5 & 4.92 & $8166 \pm 29$ & $-8065 \pm 32$ & $6505.08 \pm 0.98$ & $-5730.84 \pm 0.96$ & $255.66 \pm 0.91$ & 3,9 \\
\hline Groombridge 1830 & G7 V+ & WISEA J115302.28+374206.6 & 4.33 & $2224 \pm 59$ & $-14307 \pm 66$ & $4003.98 \pm 0.37$ & $-5813.62 \pm 0.23$ & $109.99 \pm 0.41$ & 4,9 \\
\hline Lacaille 9352 & $\mathrm{M} 2 \mathrm{~V}$ & WISEA J230557.86-355057.2 & 2.59 & $1866 \pm 56$ & $-841 \pm 60$ & $6768.20 \pm 0.59$ & $1327.52 \pm 0.68$ & $305.26 \pm 0.70$ & 5,9 \\
\hline CD-37 15492 & M3 V & WISEA J000529.35-372151.0 & 4.40 & $-471 \pm 77$ & $-2662 \pm 86$ & $5634.68 \pm 0.86$ & $-2337.71 \pm 0.71$ & $230.42 \pm 0.90$ & 5,9 \\
\hline 61 Cygni A & $\mathrm{K} 5 \mathrm{~V}$ & WISEAR J210657.70+384530.8 & 0.18 & $-4028 \pm 52$ & $5420 \pm 54$ & $4168.31 \pm 6.57$ & $3269.20 \pm 12.08$ & $286.82 \pm 6.78$ & 1,9 \\
\hline 61 Cygni B & K7 V & WISEA J2106 & 0.97 & $-1171 \pm 58$ & $215 \pm 66$ & $.90 \pm$ & 44 & $285.88 \pm$ & 1,9 \\
\hline Ross 619 & M4 V & WISEA J081158.31+084529.4 & 7.18 & $1661 \pm 34$ & $-5277 \pm 32$ & $1081.4 \pm$ & -50 & $145.5 \pm$ & 2,11 \\
\hline Teegarden's Star & M6.5 V & WISEA J025303.34+165213.2 & 7.15 & $3471 \pm 56$ & $-3666 \pm 25$ & $3403.8 \pm 2.2$ & $-3807.0 \pm 2.2$ & $260.63 \pm 2.69$ & 6,10 \\
\hline Lalande 21185 & $\mathrm{M} 2 \mathrm{~V}$ & WISEA J110319.67+355722.4 & 2.47 & $338 \pm 50$ & $786 \pm 48$ & $-580.27 \pm 0.62$ & $-4765.85 \pm 0.64$ & $392.64 \pm 0.67$ & 1,9 \\
\hline$\epsilon$ Ind A & $\mathrm{K} 2 \mathrm{~V}$ & WISEA J220326.69-564735.5 & 0.87 & $1218 \pm 32$ & $-26 \pm 36$ & $3960.93 \pm 0.24$ & $-2539.23 \pm 0.17$ & $276.06 \pm 0.28$ & 8,9 \\
\hline$\epsilon$ Ind $\mathrm{BaBb}$ & $\mathrm{T} 1+\mathrm{T} 6$ & WISEA J220415.75-564724.4 & 10.72 & $3132 \pm 41$ & $-3124 \pm 38$ & $3960.93 \pm 0.24^{b}$ & $-2539.23 \pm 0.17^{\mathrm{b}}$ & $276.06 \pm 0.28^{b}$ & 7,9 \\
\hline Wolf 359 & M6 V & WISEA J105626.19+070025.0 & 5.84 & $-2618 \pm 44$ & $-4881 \pm 42$ & $-3838.6 \pm 0.2$ & $-2697.8 \pm 0.2$ & $418.9 \pm 2.4$ & 1,12 \\
\hline
\end{tabular}

Notes. References for spectral type and high-precision astrometry: (1) Kirkpatrick et al. 1991; (2) Henry et al. 1994; (3) Keenan \& Pitts 1980; (4) Keenan \& Keller 1953; (5) Walker 1983; (6) Teegarden et al. 2003; (7) McCaughrean et al. 2004; (8) van de Kamp 1953; (9) van Leeuwen 2007; (10) Henry et al. 2006; (11) Harrington \& Dahn 1980; (12) Harrington et al. 1993.

a 61 Cygni A is the only one of these sources failing to satisfy the criteria for inclusion in the AllWISE Source Catalog. This object is part of a small-separation same-tile (SSST) source group (see Section 3.4.3). Unfortunately, despite best efforts to recover such sources, neither member of the 61 Cyg A SSST group satisfies all the criteria necessary for Catalog consideration. The entry for 61 Cyg A can, however, be found in the AllWISE Reject Table, as the source designation indicates. $\mathrm{b}$ The published astrometry for $\epsilon$ Ind $\mathrm{A}$ is used here for $\epsilon$ Ind $\mathrm{BaBb}$.

Table that have single-axis motions as large as $670^{\prime \prime} \mathrm{yr}^{-1}$ $\left(670,000\right.$ mas $\left.^{-1} r^{-1}\right)$; spot checks of these sources show them to be artifacts associated with extended structures like diffraction spikes and optical ghosts. Ultimately, the algorithm of $\chi^{2}$ minimization is only limited by the detection step on the coadd images; that is, as long as a source is identified on the coadds, extremely large motions can be recovered in the Level $1 \mathrm{~b}$ images. The only real sources that will not have a coadd detection are those moving so fast that outlier rejection will have eliminated them from the coadd entirely. Real examples of this include main belt asteroids and near-earth objects. Outlier rejection is more likely to eliminate objects seen less than $50 \%$ of the time in the frame stack. ${ }^{16}$ So it is just possible, in the two-epoch case, for an object near the ecliptic plane moving at $\sim 2.5 \times$ FWHM between the first and last exposures over one epoch, or $15^{\prime \prime}$ per $\sim 2$ days $\left(\sim 2700^{\prime \prime} \mathrm{yr}^{-1}\right)$, to have survived coaddition and be detected. For areas of sky with three epochs of coverage, assuming equal numbers of frames per epoch, a source moving at $\sim 2700^{\prime \prime} \mathrm{yr}^{-1}$ would be seen within a $2.5 \times$ FWHM window only $33 \%$ of the time, and would thus be removed from the coadd because it fails to meet the $50 \%$ outlier threshold. For such a three-epoch case, the motion limit is more likely to be $\sim 2.5 \times$ FWHM between two consecutive epochs, or $\sim 15^{\prime \prime}$ per 6 months $\left(\sim 30^{\prime \prime} \mathrm{yr}^{-1}\right)$.

\subsubsection{What is the Smallest Motion Measurable?}

Figure 5 shows the motion measurement uncertainties for sources in a typical AllWISE Atlas Image plotted against $W 1$ magnitude. Bright sources free of saturation effects $(8<W 1<10 \mathrm{mag})$ approach an asymptote of $\sim 0^{\prime} 035 \mathrm{yr}^{-1}$ in their per-axis errors. Requiring a $3 \sigma$ detection per axis means that the smallest significant motion ${ }^{17}$ that is measurable is

\footnotetext{
${ }^{16}$ See http://wise2.ipac.caltech.edu/docs/release/allsky/expsup/sec4_4f.html\# outrej of the WISE All-Sky Release Explanatory Supplement for more details.

17 Requiring the stricter $\chi^{2}$ motion $>27.63$ criterion from Section 3.4 results in a smallest motion of $\sim 0^{\prime} ! 18 \mathrm{yr}^{-1}$.
}
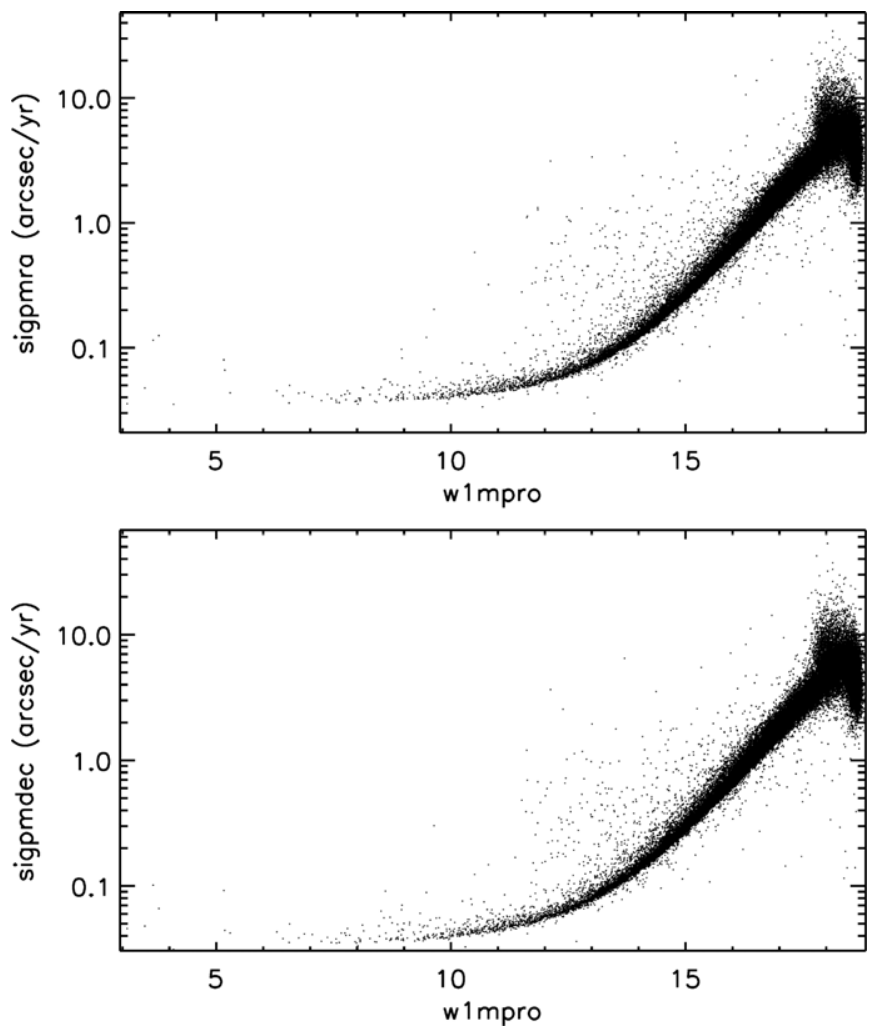

Figure 5. Uncertainty in AllWISE motion measures in R.A. (top), and Decl. (bottom) for all sources in coadd 2243m213_ac51, plotted as a function of the $W 1$ profile-fit photometry.

$\sim 0{ }^{\prime} 15 \mathrm{yr}^{-1}$. At fainter magnitudes, the smallest measurable significant motion increases dramatically as the minimum motion errors themselves increase. At $W 1=15 \mathrm{mag}$, for example, the motion errors are roughly $10 \times$ larger, meaning that the smallest measurable significant motion is $\sim 1^{\prime \prime} .5 \mathrm{yr}^{-1}$. 

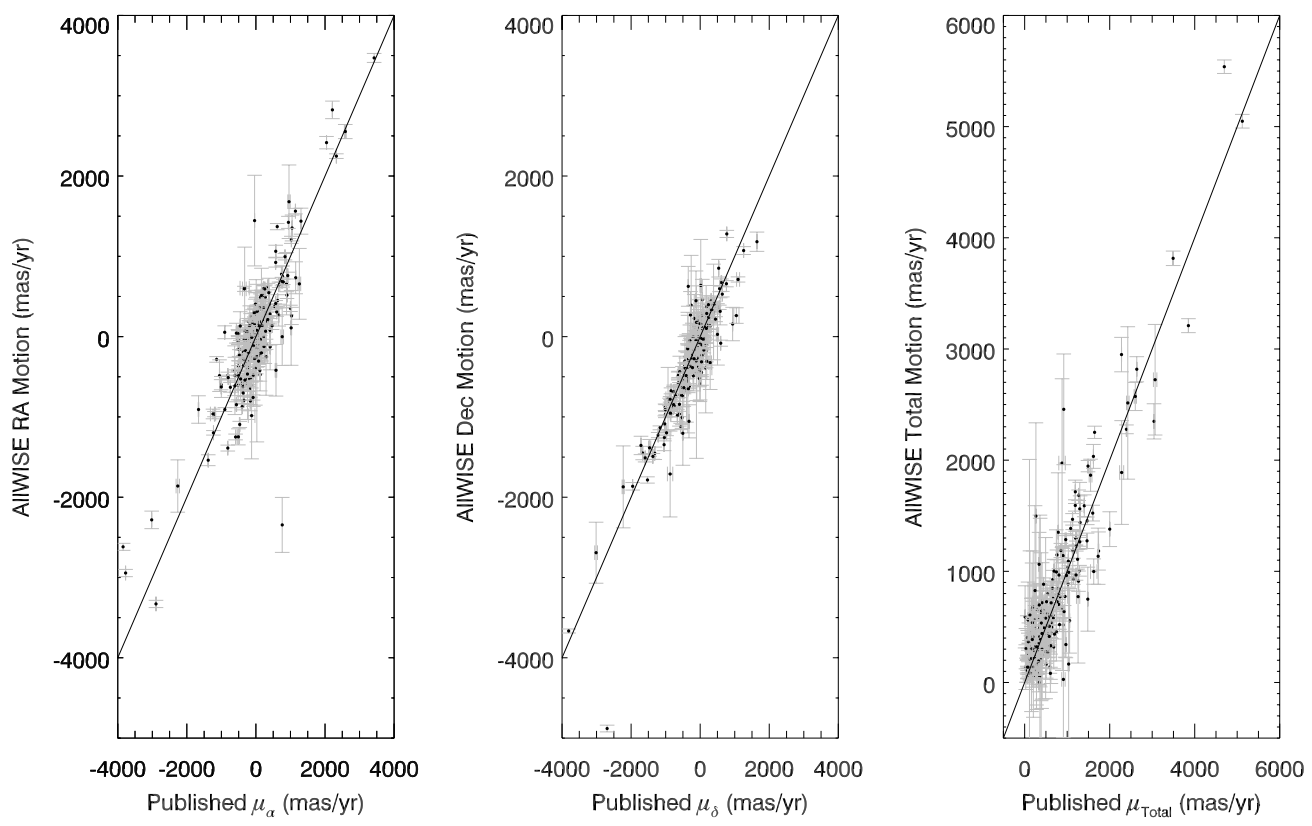

Figure 6. Comparison of the AllWISE R.A., Decl., and total motion measurements to published R.A., Decl., and total proper motion measurements of late-M, L, T, and Y dwarfs in the literature. The straight line corresponds to perfect one-to-one correspondence. The discrepant point near $(800,-2350)$ in the left panel is Wolf 940B ( $c c_{-}$flags $=$" $\left.H h 00^{\prime \prime}\right)$, which is contaminated by the halo of Wolf $940 \mathrm{~A}$, and the discrepant point near $(-2700,-4900)$ in the middle panel is the saturated star Wolf 359 (see Table 1).

The Atlas Image used in this example, 2243m213_ac51, has an average depth of coverage of $\sim 24$ framesets, as is typical of Atlas Tiles located near the ecliptic plane and observed at two WISE epochs. Atlas Tiles farther from the ecliptic will have greater coverage depths. For these cases, and for any Tiles with three WISE epochs, motion limits will be even smaller than those quoted above.

\subsection{Comparisons to Truth}

Despite the fact that AllWISE measures an apparent motion over only a short time baseline, these measurements can still be compared to proper motion values determined for stars specifically targeted in astrometric monitoring campaigns as long as the sample size is statistically significant and scattered over the entire sky so that the influence of parallax is suppressed. In the first subsection below, the AllWISE motion measurements are compared to published proper motion values for an all-sky collection of late-type dwarfs. In the second subsection, the internal repeatability of the AllWISE measurements is checked using the individual components of widely separated commonproper-motion binary systems.

\subsubsection{External Comparisons Using Previously Known Motion Objects}

A typical user of the AllWISE data products may wish to check how the AllWISE measurements compare to published values from a favorite motion catalog. As demonstrated in Section 3.1, parallax contributes to the AllWISE motion measurements, making a direct comparison difficult. Are other statistical effects seen when a large sample is used? To this end, the AllWISE measurements have been compared to published astrometry for a set of known low-mass stars and brown dwarfs. These objects were selected because they have high signalto-noise ratios in one or more WISE bands but are generally not saturated. Specifically, objects in the Database of Ultracool
Parallaxes, ${ }^{18}$ compiled as of 2013 October, were used (Dupuy \& Liu 2012). This list contains proper motions for all late-M, L, $\mathrm{T}$, and Y dwarfs that have published parallaxes. After excluding close binaries and blends, 233 objects were available for comparison. Figure 6 shows a comparison of the published values of $\mu_{\alpha}, \mu_{\delta}$, and $\mu_{\text {Total }}$ to the R.A., Decl., and total motions measured by AllWISE. The overall agreement is excellent, with the trend very closely following the line of one-to-one correspondence.

The influence of parallax on the AllWISE motion measurements can be seen in Figure 7. In this figure, the difference between the published proper motions and the AllWISE motions shows a larger dispersion in R.A. than in Decl., and both dispersions are larger than would be expected based on the average AllWISE motion errors, which are 122.3 mas and 129.4 mas for R.A. and Decl., respectively. As shown in Figure 8, which illustrates the motion differences in R.A. and Decl. plotted as a function of parallax, the R.A. differences have a striking dependence on parallax (i.e., objects with the largest differences tend to have the largest parallax values) while the Decl. differences show only a modest increase at the largest parallaxes. Because the ecliptic and equatorial systems are tilted with respect to each other by only $\sim 23$ degrees, parallactic motion, which is primarily manifested in ecliptic longitude, affects equatorial longitude (R.A.) more severely than equatorial latitude (Decl.). Other than this effect, no other issues are seen, as the R.A. and Decl. differences show a mean near zero and have no appreciable skew.

Figure 9 shows the motion difference plotted as a function of $W 2 \mathrm{mag}$, which for most $\mathrm{L}, \mathrm{T}$, and Y dwarfs is the WISE band with the highest signal-to-noise ratio and thus the band driving the astrometry. Agreement is best in the range $7<$ $W 2<12$ mag. Astrometric precision is degraded at brighter magnitudes ( $W 2<7 \mathrm{mag}$ ) because such objects have saturated image cores, and it quickly degrades at fainter magnitudes ( $W 2>12 \mathrm{mag}$ ) due to poorer photon counting statistics.

\footnotetext{
${ }^{18}$ See https://www.cfa.harvard.edu/ tdupuy/plx/Database_of_Ultracool_ Parallaxes.html.
} 

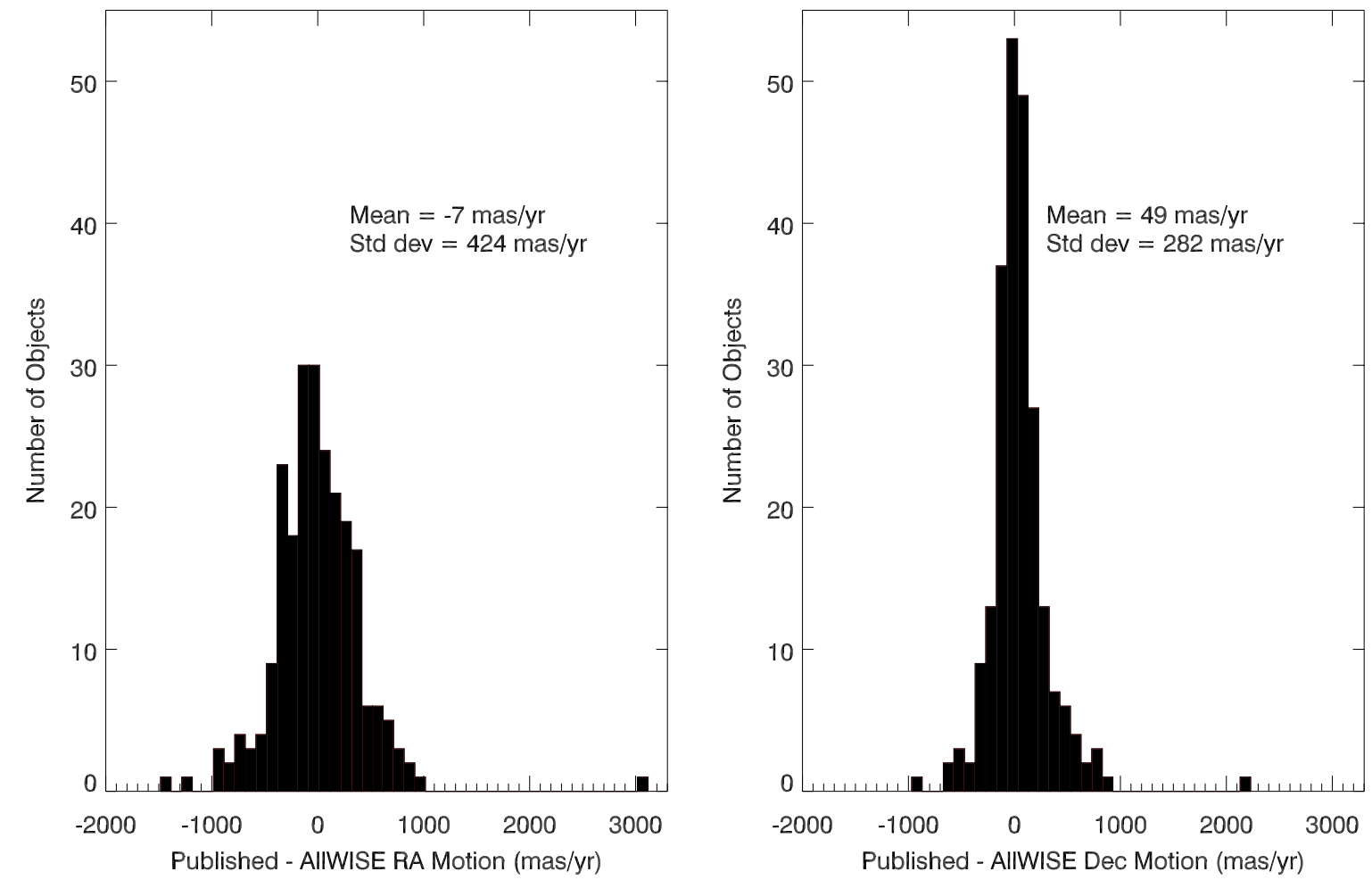

Figure 7. Histograms of the difference between published and AllWISE motions in R.A. (left panel) and Decl. (right panel) for late-M, L, T, and Y dwarfs.
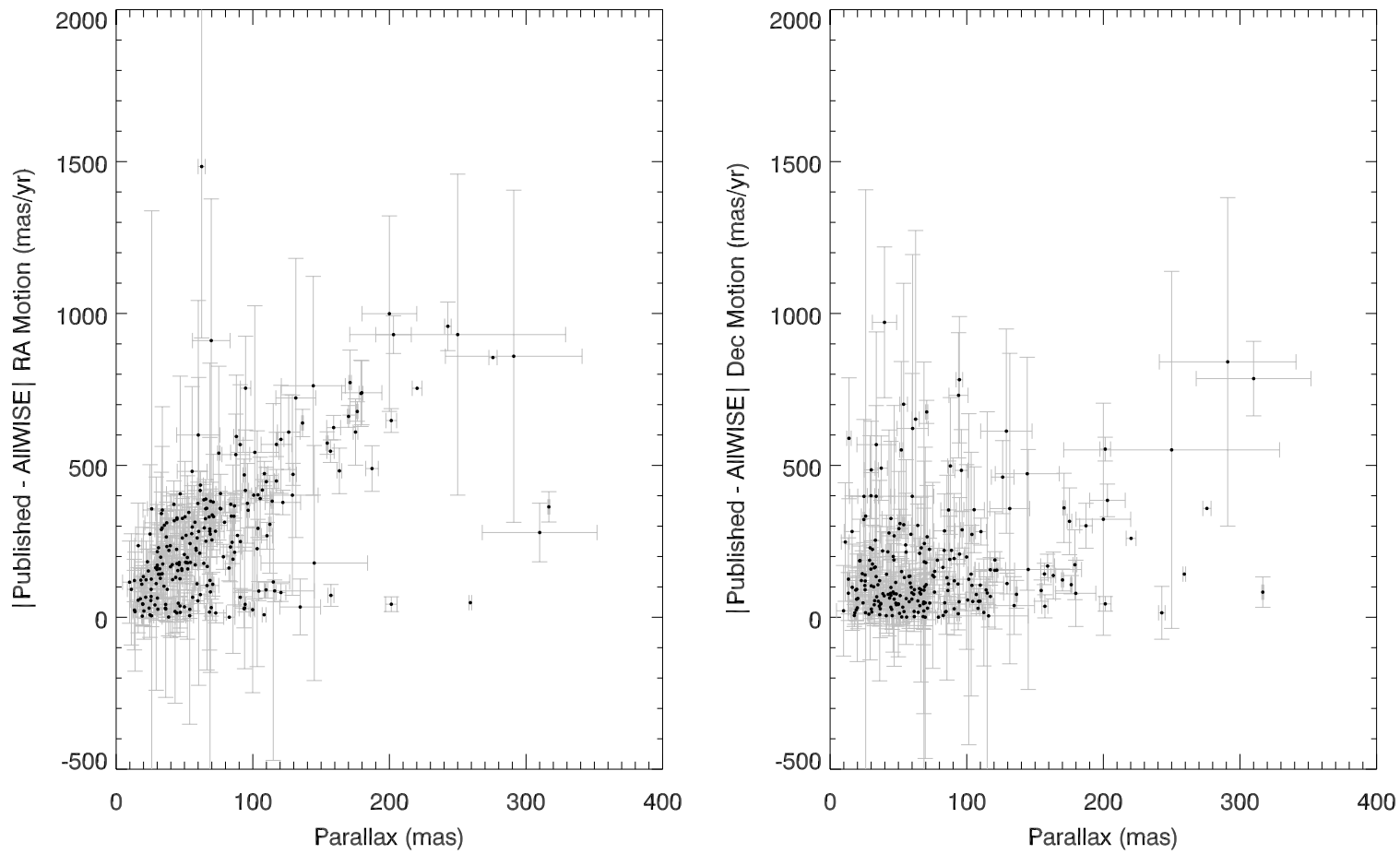

Figure 8. Absolute value of the difference between published proper motion and AllWISE motion in R.A. (left) and Decl. (right) as a function of parallax for late-M, L, T, and Y dwarfs.

\subsubsection{Internal Consistency Checks Using Common-proper-motion Binaries}

As part of AllWISE Quality Assurance checks, the software reported sources in each Atlas Tile that had a high likelihood of being real motion stars. (See Section 3.4 below.) During the course of quality assessment, reviewers noted 55 pairs of objects identified as having significant motions and located within $\sim 15^{\prime \prime}-400^{\prime \prime}$ of each other. These pairs were checked in SIMBAD and were confirmed to be known common-propermotion systems, found to be new ones verified through independent means, or identified as possible new pairs based on independent checks. The AllWISE measured motions should be identical for each member of the pair because the common parallax between components will affect the motion measures identically. Therefore, we can use these systems to perform 

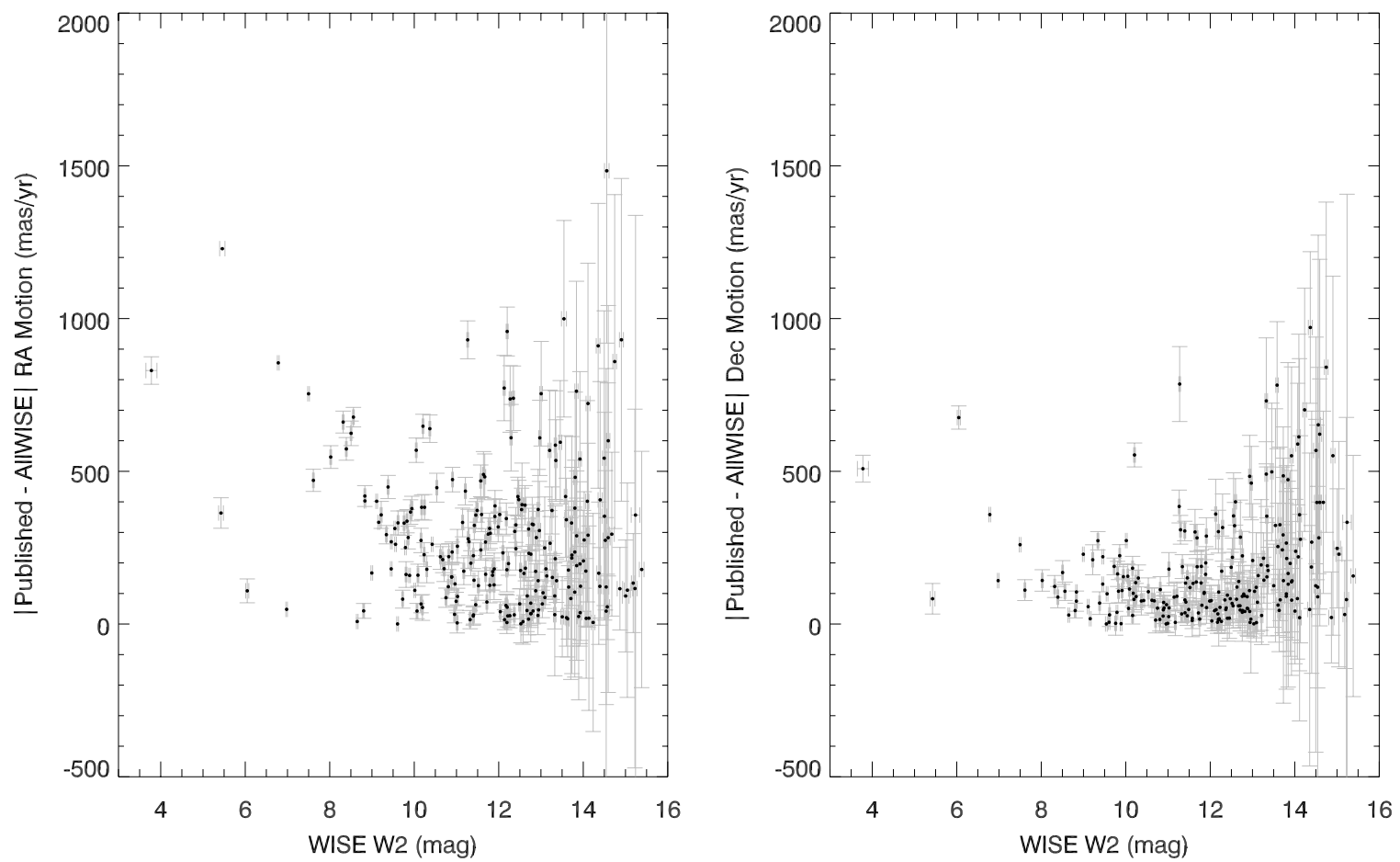

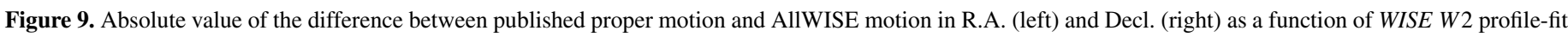
magnitude for late-M, L, T, and Y dwarfs.

an internal consistency check to see whether AllWISE motion measurements are the same within the errors.

Table 2 lists these fifty-five common-proper-motion binaries and Figure 10 shows the difference in motion between the two members of each pair. The difference is shown per axis, is expressed in units of the larger motion error within the pair, and is plotted as a function of the $W 1$ profile-fit magnitude of the source with the larger motion error. Overall, agreement is very good. The majority of unsaturated pairs has a difference $<2$ times the error.

\subsection{Motions, Both Real and False}

In this section, we examine the distribution of motions for objects in a typical $1.56 \times 1.56$ AllWISE Atlas Image. We then extract sources having statistically significant motions and discuss the average success rate for finding bona fide motion stars. In the final subsection, we take a broader view and categorize the sources of false motion seen during our quality assurance checks (described in more detail in Section 4.1) over the entire sky.

\subsubsection{A Typical Distribution of Motion Measurements}

Motion measurements for all sources in a typical Atlas Image are illustrated in Figure 11. The total proper motion is plotted as a function of $\chi^{2}$ motion $=(\text { pmra } / \text { sigpmra })^{2}+(\text { pmdec } / \text { sigpmdec })^{2}$, where pmra and pmdec are the AllWISE-measured R.A. and Decl. motions, respectively, and sigpmra and sigpmdec are their associated uncertainties. AllWISE tiles typically have $\sim 10^{5}$ extracted sources, so any source with $Q=e^{-\chi^{2}{ }_{\text {motion }} / 2}<$ $10^{-6}$ violates, ${ }^{19}$ with a very high degree of confidence, the hypothesis of a real object with zero motion (and errors correctly

\footnotetext{
19 This threshold was found to produce a sample of potentially interesting motion sources for human perusal that was not too small and not overwhelmingly large.
}
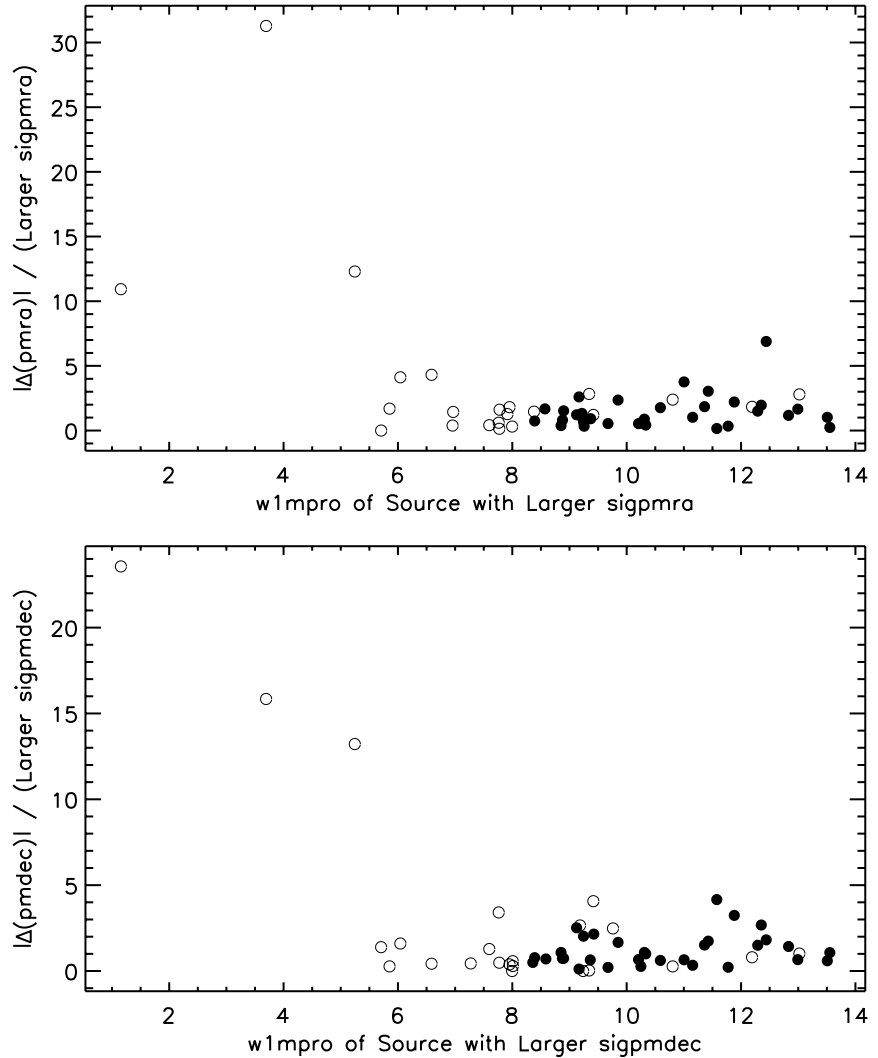

Figure 10. Difference in motion in R.A. (top) and Decl. (bottom), expressed in units of the larger motion error within the pair, for 55 common proper motion binaries noted during quality assessment. These differences are plotted as a function of the $W 1$ profile-fit magnitude of the source with the larger motion error. Pairs having at least one component in the saturated regime ( $W 1<8.1 \mathrm{mag}$ or $W 2<7.0 \mathrm{mag}$ ) are shown by open circles. All others are shown by solid circles. 
Table 2

Common-proper-motion Binaries Noted During AllWISE Quality Checks

\begin{tabular}{|c|c|c|c|c|c|}
\hline WISEA Designation & $\begin{array}{c}W 1 \\
(\mathrm{mag})\end{array}$ & $\begin{array}{c}W 2 \\
(\mathrm{mag}) \\
(3)\end{array}$ & $\begin{array}{c}\text { AllWISE } \\
\text { R.A. Motion } \\
\left(\mathrm{mas} \mathrm{yr}^{-1}\right) \\
(4)\end{array}$ & $\begin{array}{c}\text { AllWISE } \\
\text { Decl. Motion } \\
\left(\mathrm{mas} \mathrm{yr}^{-1}\right) \\
(5)\end{array}$ & Note \\
\hline $\mathrm{J} 012655.16+120022.0$ & $9.872 \pm 0.022$ & $9.886 \pm 0.021$ & $-83 \pm 36$ & $-364 \pm 33$ & NLTT 4817, LSPM J0126+1200N \\
\hline J012654.11+120002.9 & $12.830 \pm 0.023$ & $12.669 \pm 0.027$ & $-151 \pm 58$ & $-444 \pm 56$ & NLTT 4814, LSPM J0126+1200S (sep 24".5) \\
\hline J013054.96+524442.3 & $7.915 \pm 0.027$ & $7.977 \pm 0.020$ & $-222 \pm 26$ & $22 \pm 24$ & $\mathrm{BD}+51318 \mathrm{~A}$ \\
\hline J013056.36+524500.8 & $9.760 \pm 0.024$ & $9.658 \pm 0.020$ & $-189 \pm 25$ & $-40 \pm 25$ & $\mathrm{BD}+51318 \mathrm{~B}\left(\operatorname{sep} 22^{\prime \prime} .5\right)$ \\
\hline J015329.15+732940.2 & $8.109 \pm 0.023$ & $8.058 \pm 0.020$ & $420 \pm 31$ & $-208 \pm 30$ & LP 29-233, "LHS 6036" \\
\hline J015326.94+733015.4 & $9.124 \pm 0.023$ & $8.939 \pm 0.020$ & $459 \pm 32$ & $-130 \pm 31$ & LP 29-232, “LHS 6035” (sep 36".4) \\
\hline J021157.87+042140.9 & $6.955 \pm 0.054$ & $7.020 \pm 0.020$ & $-155 \pm 26$ & $-11 \pm 25$ & BD+03 301, LSPM J0211+0421 \\
\hline J021159.43+042150.7 & $9.184 \pm 0.023$ & $9.080 \pm 0.020$ & $-145 \pm 26$ & $-80 \pm 26$ & new cpm companion ${ }^{\mathrm{a}}\left(\mathrm{sep} 25^{\prime \prime} \cdot 3\right)$ \\
\hline J022104.69+365258.7 & $8.358 \pm 0.024$ & $8.180 \pm 0.021$ & $768 \pm 26$ & $-562 \pm 26$ & GJ 1047AB, LHS 1393 \\
\hline J022102.54+365241.5 & $9.256 \pm 0.023$ & $9.060 \pm 0.020$ & $777 \pm 27$ & $-549 \pm 26$ & GJ 1047C, LHS 1392 (sep 31".0) \\
\hline J025051.72+033035.6 & $8.852 \pm 0.022$ & $8.904 \pm 0.019$ & $175 \pm 26$ & $-83 \pm 25$ & BD+02 436A, LSPM J0250+0330N \\
\hline J025051.32+033006.7 & $9.023 \pm 0.022$ & $9.077 \pm 0.019$ & $185 \pm 26$ & $-56 \pm 25$ & BD+02 436B, LSPM J0250+0330S (sep 29'.5) \\
\hline J025403.21-355418.7 & $5.853 \pm 0.119$ & $5.731 \pm 0.047$ & $523 \pm 26$ & $-150 \pm 26$ & LHS 1466 \\
\hline J025402.74-355454.7 & $8.179 \pm 0.022$ & $8.066 \pm 0.019$ & $479 \pm 24$ & $-143 \pm 24$ & LHS 1467 (sep 36".4) \\
\hline J030531.36+114952.8 & $7.997 \pm 0.023$ & $7.863 \pm 0.019$ & $165 \pm 26$ & $-254 \pm 26$ & G 5-13, LSPM J0305+1149N \\
\hline J030530.76+114932.1 & $8.346 \pm 0.023$ & $8.249 \pm 0.020$ & $157 \pm 26$ & $-254 \pm 26$ & G 5-12, LSPM J0305+1149S (sep 22".6) \\
\hline J031407.66+083319.0 & $8.391 \pm 0.023$ & $8.433 \pm 0.021$ & $303 \pm 38$ & $-14 \pm 36$ & G 79-15, LSPM J0314+0833S \\
\hline J031408.29+083345.6 & $8.707 \pm 0.022$ & $8.702 \pm 0.021$ & $275 \pm 37$ & $14 \pm 36$ & G 79-16, LSPM J0314+0833N (sep 28".2) \\
\hline J031608.21-374158.4 & $9.565 \pm 0.023$ & $9.624 \pm 0.020$ & $65 \pm 24$ & $51 \pm 24$ & TYC 7561-77-1 \\
\hline J031606.54-374215.4 & $13.552 \pm 0.024$ & $13.341 \pm 0.027$ & $55 \pm 42$ & $6 \pm 42$ & new cpm companion ${ }^{\mathrm{b}}\left(\mathrm{sep} 26^{\prime \prime} 1\right)$ \\
\hline J033528.69-321806.2 & $9.007 \pm 0.023$ & $8.941 \pm 0.020$ & $-292 \pm 25$ & $-331 \pm 24$ & LP 888-32, LEHPM 3410 \\
\hline J033530.11-321829.1 & $10.333 \pm 0.023$ & $10.146 \pm 0.021$ & $-303 \pm 26$ & $-306 \pm 25$ & LP 888-33, LEHPM 3411 (sep 29!.1) \\
\hline J033936.37+252814.8 & $7.777 \pm 0.024$ & $7.698 \pm 0.020$ & $377 \pm 36$ & $-497 \pm 34$ & Wolf 204, LHS 1573 \\
\hline J033940.66+252842.2 & $8.007 \pm 0.023$ & $7.919 \pm 0.021$ & $319 \pm 36$ & $-517 \pm 35$ & Wolf 205, LHS 1574 (sep 64."4) \\
\hline J035228.33-315026.6 & $12.741 \pm 0.023$ & $12.499 \pm 0.023$ & $231 \pm 32$ & $-531 \pm 32$ & LHS 1609 \\
\hline J035227.56-315105.2 & $13.508 \pm 0.023$ & $13.254 \pm 0.026$ & $189 \pm 41$ & $-506 \pm 42$ & new cpm companion ${ }^{\mathrm{c}}$ (sep 39!'8) \\
\hline J043942.59+095215.6 & $6.464 \pm 0.086$ & $6.406 \pm 0.023$ & $-24 \pm 37$ & $-456 \pm 35$ & G $83-28$ \\
\hline J043943.24+095142.9 & $9.238 \pm 0.023$ & $9.077 \pm 0.020$ & $14 \pm 39$ & $-456 \pm 37$ & G 83-29 (sep 34".1) \\
\hline J044349.59-481934.0 & $5.706 \pm 0.130$ & $5.558 \pm 0.046$ & $63 \pm 37$ & $347 \pm 36$ & LTT 2077 \\
\hline J044350.89-482003.8 & $9.517 \pm 0.023$ & $9.363 \pm 0.020$ & $63 \pm 31$ & $297 \pm 30$ & new cpm companion ${ }^{\mathrm{d}}\left(\mathrm{sep} 32^{\prime \prime} .5\right)$ \\
\hline J051729.18-345346.0 & $>1.155$ & $>1.308$ & $712 \pm 42^{y}$ & $627 \pm 39^{y}$ & $o$ Columbae, $\mathrm{CD}-352214$ \\
\hline J051723.88-345121.8 & $9.724 \pm 0.023$ & $9.538 \pm 0.019$ & $253 \pm 34$ & $-292 \pm 33$ & new cpm companion ${ }^{\mathrm{e}}\left(\mathrm{sep} 158^{\prime \prime 2} 2\right)$ \\
\hline J065438.74+131038.5 & $3.694 \pm 0.394$ & $3.369 \pm 0.283$ & $2033 \pm 57^{y}$ & $-1035 \pm 64^{y}$ & 38 Geminorum AB, HR 2564 \\
\hline J065448.82+131002.5 & $9.840 \pm 0.023$ & $9.664 \pm 0.020$ & $250 \pm 38$ & $-21 \pm 36$ & new cpm companion (sep 151".6) $^{\prime \prime}$ \\
\hline J074020.22-172451.5 & $9.064 \pm 0.022$ & $8.831 \pm 0.020$ & $1563 \pm 34$ & $-628 \pm 33$ & LHS 235 \\
\hline J074021.62-172454.8 & $12.440 \pm 0.023$ & $12.432 \pm 0.025$ & $1928 \pm 53$ & $-726 \pm 54$ & LHS $234^{\mathrm{g}}\left(\operatorname{sep} 20^{\prime \prime} 3\right)$ \\
\hline J075812.35+875734.7 & $8.850 \pm 0.022$ & $8.681 \pm 0.020$ & $-222 \pm 28$ & $-536 \pm 28$ & LHS 1962 \\
\hline J075909.31+875742.9 & $10.207 \pm 0.023$ & $10.001 \pm 0.020$ & $-238 \pm 30$ & $-556 \pm 30$ & LHS 1965 (sep 31".5) \\
\hline J082854.03-243541.8 & $9.308 \pm 0.022$ & $9.185 \pm 0.021$ & $200 \pm 36$ & $-335 \pm 36$ & WT 1549 \\
\hline J082852.10-243536.5 & $11.881 \pm 0.023$ & $11.665 \pm 0.022$ & $297 \pm 44$ & $-186 \pm 46$ & new cpm companion ${ }^{\mathrm{h}}\left(\mathrm{sep} 26^{\prime \prime} .9\right)$ \\
\hline J103734.02+295955.5 & $10.092 \pm 0.023$ & $9.939 \pm 0.021$ & $284 \pm 38$ & $-84 \pm 38$ & LP 316-395, LSPM J1037+2959N \\
\hline J103734.43+295937.9 & $11.153 \pm 0.022$ & $10.967 \pm 0.022$ & $327 \pm 42$ & $-98 \pm 42$ & LP 316-396, LSPM J1037+2959S (sep 18".4) \\
\hline J105607.88-575041.1 & $10.309 \pm 0.022$ & $10.164 \pm 0.020$ & $-182 \pm 25$ & $125 \pm 25$ & UPM J1056-5750 \\
\hline J105536.09-575042.1 & $10.366 \pm 0.023$ & $10.227 \pm 0.020$ & $-160 \pm 24$ & $152 \pm 25$ & new cpm companion ${ }^{\mathrm{i}}\left(\mathrm{sep} 254^{\prime \prime} 8\right)$ \\
\hline J110604.58+425246.3 & $10.323 \pm 0.023$ & $10.151 \pm 0.020$ & $-156 \pm 35$ & $-384 \pm 36$ & G 176-13 \\
\hline J110605.07+425303.3 & $11.361 \pm 0.023$ & $11.111 \pm 0.021$ & $-84 \pm 39$ & $-325 \pm 39$ & G 176-14 (sep 17".9) \\
\hline J113333.55-413954.4 & $8.675 \pm 0.023$ & $8.712 \pm 0.020$ & $-140 \pm 25$ & $-134 \pm 25$ & CD-40 6796 \\
\hline J113333.67-414016.7 & $10.589 \pm 0.024$ & $10.484 \pm 0.020$ & $-186 \pm 26$ & $-118 \pm 26$ & new cpm companion ${ }^{\mathrm{j}}\left(\mathrm{sep} 22^{\prime \prime} .3\right)$ \\
\hline $\mathrm{J} 120907.21+473602.2$ & $9.166 \pm 0.023$ & $8.975 \pm 0.021$ & $713 \pm 35$ & $-349 \pm 35$ & LHS 2516 \\
\hline J120907.24+473531.8 & $10.036 \pm 0.024$ & $9.856 \pm 0.020$ & $804 \pm 35$ & $-353 \pm 35$ & LHS 2517 (sep 30."4) \\
\hline J121058.01-461917.3 & $6.042 \pm 0.097$ & $5.997 \pm 0.040$ & $-4 \pm 26$ & $169 \pm 25$ & CD-45 7595, LTT 4560 \\
\hline J121058.26-461204.5 & $8.822 \pm 0.022$ & $8.711 \pm 0.020$ & $103 \pm 25$ & $129 \pm 25$ & new cpm companion ${ }^{\mathrm{k}}\left(\mathrm{sep} 432^{\prime \prime} 8\right)$ \\
\hline J124007.18+204828.9 & $6.801 \pm 0.066$ & $6.837 \pm 0.020$ & $250 \pm 35$ & $-449 \pm 35$ & G 59-32, BD+21 2442 \\
\hline J124014.80+204752.7 & $13.020 \pm 0.024$ & $12.803 \pm 0.026$ & $426 \pm 63$ & $-381 \pm 67$ & new cpm companion ${ }^{1}$ (sep 112.7) \\
\hline J124725.86-434353.2 & $7.850 \pm 0.023$ & $7.893 \pm 0.019$ & $-165 \pm 24$ & $-162 \pm 24$ & LTT 4892, CD-43 7881 \\
\hline J124726.75-434441.8 & $12.192 \pm 0.023$ & $11.997 \pm 0.021$ & $-220 \pm 30$ & $-138 \pm 30$ & new cpm companion ${ }^{\mathrm{m}}(\mathrm{sep} 49 \cdot 5)$ \\
\hline J124737.85-274637.3 & $9.715 \pm 0.022$ & $9.568 \pm 0.020$ & $-220 \pm 27$ & $-15 \pm 26$ & LP 853-37 \\
\hline J124739.94-274642.7 & $9.849 \pm 0.022$ & $9.700 \pm 0.020$ & $-154 \pm 28$ & $-60 \pm 27$ & LP 853-38 (sep 28."4) \\
\hline J125433.93-381122.7 & $7.951 \pm 0.024$ & $8.016 \pm 0.020$ & $208 \pm 26$ & $-68 \pm 26$ & TYC 7772-1225-1 \\
\hline J125435.54-381111.7 & $8.175 \pm 0.022$ & $8.209 \pm 0.019$ & $161 \pm 26$ & $-78 \pm 26$ & possible new cpm companion $^{\mathrm{n}}\left(\mathrm{sep} 22^{\prime \prime} .0\right.$ ) \\
\hline $\mathrm{J} 131407.63+061814.3$ & $11.804 \pm 0.022$ & $11.790 \pm 0.022$ & $-206 \pm 33$ & $-294 \pm 33$ & G 62-19, LSPM J1314+0618S \\
\hline J131408.36+061828.3 & $12.291 \pm 0.022$ & $12.231 \pm 0.022$ & $-152 \pm 36$ & $-240 \pm 36$ & G 62-20, LSPM J1314+0618N (sep 17".8) \\
\hline J134454.85-453518.5 & $7.547 \pm 0.025$ & $7.570 \pm 0.019$ & $-304 \pm 33$ & $67 \pm 32$ & LTT 5330 \\
\hline J134457.17-453537.2 & $9.343 \pm 0.022$ & $9.160 \pm 0.019$ & $-403 \pm 35$ & $68 \pm 34$ & new cpm companion ${ }^{\circ}\left(\operatorname{sep} 30^{\prime \prime} 7\right)$ \\
\hline
\end{tabular}


Table 2

(Continued)

\begin{tabular}{|c|c|c|c|c|c|}
\hline WISEA Designation & $\begin{array}{c}W 1 \\
\text { (mag) }\end{array}$ & $\begin{array}{c}W 2 \\
\text { (mag) }\end{array}$ & $\begin{array}{c}\text { AllWISE } \\
\text { R.A. Motion } \\
\left(\operatorname{mas~yr}^{-1}\right) \\
(4)\end{array}$ & $\begin{array}{c}\text { AllWISE } \\
\text { Decl. Motion } \\
\left(\mathrm{mas} \mathrm{yr}^{-1}\right) \\
(5)\end{array}$ & Note \\
\hline J140400.30-592400.5 & $9.363 \pm 0.021$ & $9.179 \pm 0.020$ & $-44 \pm 34$ & $-418 \pm 34$ & L 197-165, NLTT 36089 \\
\hline J140350.20-592348.0 & $9.371 \pm 0.022$ & $9.194 \pm 0.020$ & $-76 \pm 35$ & $-396 \pm 34$ & new cpm companion ${ }^{\mathrm{p}}\left(\operatorname{sep} 78^{\prime \prime} .1\right)$ \\
\hline J140926.79-305551.4 & $7.772 \pm 0.026$ & $7.819 \pm 0.021$ & $-496 \pm 29$ & $-271 \pm 29$ & LHS 2807 \\
\hline J140924.56-305534.1 & $8.728 \pm 0.023$ & $8.643 \pm 0.020$ & $-492 \pm 26$ & $-285 \pm 25$ & new cpm companion ${ }^{\mathrm{q}}\left(\mathrm{sep} 33^{\prime \prime} 4\right)$ \\
\hline J141509.28+221537.3 & $8.877 \pm 0.022$ & $8.891 \pm 0.020$ & $-212 \pm 26$ & $129 \pm 26$ & NLTT 36714, LSPM J1415+2215S \\
\hline J141509.80+221559.8 & $8.966 \pm 0.022$ & $9.042 \pm 0.019$ & $-191 \pm 26$ & $110 \pm 26$ & NLTT 36716, LSPM J1415+2215N (sep 23".7) \\
\hline J142051.70-045805.5 & $8.348 \pm 0.032$ & $8.393 \pm 0.029$ & $-76 \pm 25$ & $-75 \pm 26$ & BD-04 3668 \\
\hline J142053.60-050137.8 & $11.003 \pm 0.023$ & $10.877 \pm 0.020$ & $-185 \pm 29$ & $-56 \pm 29$ & new cpm companion ${ }^{\mathrm{r}}\left(\operatorname{sep} 214^{\prime \prime} 2\right)$ \\
\hline J153519.64+174245.2 & $7.762 \pm 0.022$ & $7.597 \pm 0.019$ & $-1455 \pm 38$ & $34 \pm 34$ & Ross 513A, LHS 399 \\
\hline J153519.45+174302.6 & $9.316 \pm 0.023$ & $9.081 \pm 0.020$ & $-1478 \pm 36$ & $-82 \pm 33$ & Ross 513B, LHS 400 (sep 17".6) \\
\hline J154850.51+175056.1 & $8.855 \pm 0.023$ & $8.781 \pm 0.020$ & $-268 \pm 36$ & $165 \pm 36$ & G 137-55, LSPM J1548+1750E \\
\hline $\mathrm{J} 154848.52+175050.0$ & $9.673 \pm 0.022$ & $9.525 \pm 0.020$ & $-288 \pm 37$ & $157 \pm 38$ & G 137-54, LSPM J1548+1750W (sep 29!'1) \\
\hline J155306.56+344508.8 & $6.966 \pm 0.034$ & $6.877 \pm 0.020$ & $158 \pm 32$ & $-470 \pm 29$ & LHS 3129 \\
\hline J155306.84+344442.4 & $8.009 \pm 0.023$ & $7.884 \pm 0.020$ & $112 \pm 32$ & $-479 \pm 31$ & LHS 3130 (sep 26".6) \\
\hline J155831.76+572242.3 & $7.597 \pm 0.030$ & $7.674 \pm 0.020$ & $133 \pm 24$ & $-241 \pm 25$ & G 225-39 \\
\hline J155831.67+572309.1 & $10.000 \pm 0.023$ & $9.823 \pm 0.020$ & $143 \pm 24$ & $-209 \pm 24$ & G 225-38 (sep 26."8) \\
\hline J164836.25+550743.4 & $7.273 \pm 0.030$ & $7.325 \pm 0.020$ & $162 \pm 25$ & $-269 \pm 25$ & G 226-28 \\
\hline J164835.74+550809.7 & $8.379 \pm 0.023$ & $8.461 \pm 0.020$ & $124 \pm 26$ & $-258 \pm 25$ & G 226-27 (sep 26".6) \\
\hline J165524.66-081930.5 & $6.588 \pm 0.061$ & $6.374 \pm 0.022$ & $-1207 \pm 42$ & $-764 \pm 40$ & LHS 427 \\
\hline J165534.68-082349.7 & $8.619 \pm 0.023$ & $8.393 \pm 0.020$ & $-1388 \pm 37$ & $-781 \pm 35$ & LHS 429, vB 8 (sep 298.'9) \\
\hline J170428.76+034342.7 & $8.570 \pm 0.022$ & $8.586 \pm 0.020$ & $-408 \pm 37$ & $-196 \pm 36$ & G $19-10$ \\
\hline J170428.57+034422.7 & $8.587 \pm 0.023$ & $8.648 \pm 0.020$ & $-346 \pm 37$ & $-170 \pm 37$ & G 19-11 (sep 40'.1) \\
\hline J171828.99-224630.2 & $9.217 \pm 0.024$ & $9.056 \pm 0.020$ & $-270 \pm 43$ & $-138 \pm 39$ & new cpm system ${ }^{\mathrm{s}}$ \\
\hline J171826.98-224543.5 & $9.424 \pm 0.023$ & $9.313 \pm 0.019$ & $-327 \pm 42$ & $-52 \pm 40$ & new cpm system ${ }^{\mathrm{s}}$ (sep 54.3) \\
\hline J172230.07-695119.2 & $8.336 \pm 0.023$ & $8.243 \pm 0.020$ & $-236 \pm 40$ & $-221 \pm 36$ & possible new cpm system ${ }^{\mathrm{t}}$ \\
\hline J172237.14-695112.2 & $11.426 \pm 0.023$ & $11.259 \pm 0.021$ & $-81 \pm 51$ & $-308 \pm 50$ & possible new cpm system ${ }^{\mathrm{t}}\left(\mathrm{sep} 54^{\prime \prime} .3\right)$ \\
\hline J182000.63-522138.6 & $9.244 \pm 0.023$ & $9.170 \pm 0.020$ & $125 \pm 39$ & $-308 \pm 38$ & L 272-87, NLTT 46256 \\
\hline J182001.39-522200.7 & $9.512 \pm 0.023$ & $9.399 \pm 0.020$ & $151 \pm 38$ & $-385 \pm 38$ & L 272-88, NLTT 46257 (sep 23."1) \\
\hline J182038.54+404836.0 & $11.156 \pm 0.023$ & $11.047 \pm 0.020$ & $-202 \pm 39$ & $-264 \pm 42$ & NLTT 46342, LSPM J1820+4048N \\
\hline $\mathrm{J} 182038.57+404759.2$ & $11.774 \pm 0.023$ & $11.609 \pm 0.020$ & $-188 \pm 41$ & $-274 \pm 46$ & NLTT 46343, LSPM J1820+4048S (sep 36."9) \\
\hline J185252.01-570745.3 & $7.473 \pm 0.032$ & $7.462 \pm 0.020$ & $-368 \pm 35$ & $-836 \pm 34$ & LHS 3421 \\
\hline J185257.45-570821.9 & $10.804 \pm 0.024$ & $10.620 \pm 0.020$ & $-466 \pm 41$ & $-847 \pm 41$ & 2MASS J18525777-5708141 (sep 57".4) \\
\hline J190250.67-755058.1 & $10.928 \pm 0.023$ & $10.768 \pm 0.021$ & $-13 \pm 38$ & $-225 \pm 38$ & SCR J1902-7550A \\
\hline J190254.95-755110.8 & $12.353 \pm 0.023$ & $12.135 \pm 0.023$ & $87 \pm 51$ & $-375 \pm 56$ & SCR J1902-7550B (sep 22".5) \\
\hline J201605.71-115838.5 & $8.778 \pm 0.022$ & $8.629 \pm 0.019$ & $-258 \pm 39$ & $-230 \pm 37$ & new cpm system ${ }^{\mathrm{u}}$ \\
\hline J201605.96-115916.7 & $8.897 \pm 0.022$ & $8.746 \pm 0.019$ & $-197 \pm 40$ & $-202 \pm 38$ & new cpm system ${ }^{\mathrm{u}}\left(\mathrm{sep} 38^{\prime \prime} 3\right)$ \\
\hline J205103.71-013357.8 & $9.639 \pm 0.023$ & $9.611 \pm 0.019$ & $-285 \pm 41$ & $-168 \pm 40$ & Wolf 885 , NLTT 50000 \\
\hline J205103.50-013342.6 & $10.248 \pm 0.023$ & $10.136 \pm 0.019$ & $-310 \pm 43$ & $-179 \pm 41$ & Wolf 884, NLTT 49999 (sep 15"6) \\
\hline J210630.56-382606.1 & $12.874 \pm 0.023$ & $12.599 \pm 0.026$ & $156 \pm 67$ & $-530 \pm 69$ & new cpm system ${ }^{\mathrm{V}}$ \\
\hline J210632.73-382640.4 & $12.992 \pm 0.023$ & $12.717 \pm 0.025$ & $40 \pm 70$ & $-577 \pm 71$ & new cpm system ${ }^{v}\left(\operatorname{sep} 42^{\prime \prime} 7\right)$ \\
\hline J214737.18-135422.0 & $9.809 \pm 0.023$ & $9.798 \pm 0.020$ & $156 \pm 41$ & $-260 \pm 42$ & Ross 208 \\
\hline J214736.41-135335.4 & $11.575 \pm 0.023$ & $11.464 \pm 0.021$ & $164 \pm 51$ & $-468 \pm 50$ & new cpm companion ${ }^{\mathrm{w}}\left(\mathrm{sep} 48^{\prime \prime} 0\right)$ \\
\hline $\mathrm{J} 230213.97+801412.0$ & $6.683 \pm 0.071$ & $6.704 \pm 0.022$ & $-73 \pm 27$ & $168 \pm 28$ & BD+79 762 \\
\hline J230226.16+801241.8 & $9.417 \pm 0.023$ & $9.278 \pm 0.020$ & $-108 \pm 29$ & $290 \pm 30$ & new cpm companion ${ }^{\mathrm{x}}\left(\mathrm{sep} 95^{\prime \prime} 3\right)$ \\
\hline J231049.97+453041.3 & $5.245 \pm 0.174$ & $5.107 \pm 0.072$ & $-864 \pm 54^{y}$ & $331 \pm 55^{y}$ & HD 218868, LTT 16813, WDS 23108+4531A \\
\hline $\mathrm{J} 231054.77+453043.5$ & $9.690 \pm 0.023$ & $9.515 \pm 0.021$ & $-200 \pm 36$ & $-396 \pm 35$ & WDS 23108+4531C (sep 50'.5) \\
\hline
\end{tabular}

Notes.

${ }^{a}$ WISEA J021159.43+042150.7: Using the 2MASS-to-WISE time baseline we obtain a motion, $\left(\mu_{\alpha}, \mu_{\delta}\right)$, of $(-140.5 \pm 10.2,-65.8 \pm 9.3)$ mas yr ${ }^{-1}$ for this object. The other component, BD+03 301, has a published motion of $(-144.70 \pm 1.64,-78.66 \pm 1.18)$ mas $\mathrm{yr}^{-1}$ (van Leeuwen 2007). These motions are identical within their $2 \sigma$ errors, so this is likely a common-proper-motion system.

${ }^{\mathrm{b}}$ WISEA J031606.54-374215.4: Using the 2MASS-to-WISE time baseline we obtain a motion, $\left(\mu_{\alpha}, \mu_{\delta}\right)$, of $(48.0 \pm 11.3,36.3 \pm 10.4) \mathrm{mas}^{-1}$ for this object. The other component, TYC 7561-77-1, has a published motion of (57.4 $\pm 2.2,30.6 \pm 2.1)$ mas yr$^{-1}$ (Høg et al. 2000). Although small, these motions are identical within their $1 \sigma$ errors, so this is likely a common-proper-motion system.

${ }^{c}$ WISEA J035227.56-315105.2: Using the 2MASS-to-WISE time baseline we obtain a motion, $\left(\mu_{\alpha}, \mu_{\delta}\right)$, of $(196.6 \pm 11.0,-531.9 \pm 10.4) \mathrm{mas}^{-1}$ for this

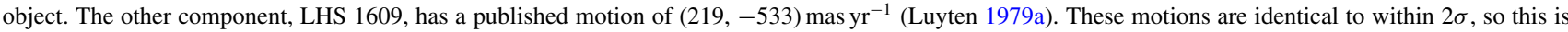
likely a common-proper-motion binary.

${ }^{\mathrm{d}}$ WISEA J044350.89-482003.8: Using the 2MASS-to-WISE time baseline we obtain a motion, $\left(\mu_{\alpha}, \mu_{\delta}\right)$, of $(-5.5 \pm 9.0,263.4 \pm 8.8) \mathrm{mas}^{-1}$ for this object. The other component, LTT 2077, has a published motion of $(4.75 \pm 0.37,276.85 \pm 0.43)$ mas $^{-1} r^{-1}$ (van Leeuwen 2007). These motions are identical to within $2 \sigma$, so this is likely a common-proper-motion binary.

${ }^{\mathrm{e}}$ WISEA J051723.88-345121.8: Using the 2MASS-to-WISE time baseline we obtain a motion, $\left(\mu_{\alpha}, \mu_{\delta}\right)$, of $(86.7 \pm 8.2,-340.4 \pm 8.0)$ mas yr $^{-1}$ for this object.

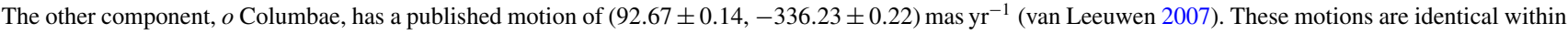
their $1 \sigma$ errors, so this is very likely a common-proper-motion system. 
${ }^{\mathrm{f}}$ WISEA J065448.82+131002.5: Using the 2MASS-to-WISE time baseline we obtain a motion, $\left(\mu_{\alpha}, \mu_{\delta}\right)$, of $(63.7 \pm 9.2,-86.2 \pm 8.8)$ mas yr ${ }^{-1}$ for this object. The other component, 38 Geminorum $\mathrm{AB}$, has a published motion of $(68.48 \pm 1.78,-72.98 \pm 1.46)$ mas yr$^{-1}$ (van Leeuwen 2007). These motions are identical within their $2 \sigma$ errors, so this is likely a common-proper-motion system.

g WISEA J074021.62-172454.8: This object falls in a tile overlap region, so it was processed twice by AllWISE. The apparition chosen for inclusion in the AllWISE Source Catalog, whose values are listed in the table above, was selected over the other apparition because the former is further from the tile edge. The other apparition, which appears in the AllWISE Reject Table as WISEAR J074021.62-172454.8, has motion values of $1563 \pm 50 \mathrm{mas}^{-1}$ and $-608 \pm 52 \mathrm{mas} \mathrm{yr}^{-1}$ in R.A. and Decl., respectively. The values for the AllWISE Reject source are $<1 \sigma$ discrepant from either apparition of the primary, LHS 235. Because the same underlying frames are used for both tiles, it is unclear why the motion for one of the apparitions of LHS 234 is so wildly discrepant in R.A.

${ }^{\mathrm{h}}$ WISEA J082852.10-243536.5: Using the 2MASS-to-WISE time baseline we obtain a motion, $\left(\mu_{\alpha}, \mu_{\delta}\right)$, of $(157.6 \pm 10.8,-137.5 \pm 10.7) \mathrm{mas}^{-1}$ for this object. The other component, WT 1549, has a published motion of $(151 \pm 13,-140 \pm 13)$ mas yr $^{-1}$ (Wroblewski \& Torres 1996). These motions are identical within their $1 \sigma$ errors, so this is very likely a common-proper-motion system.

${ }^{\mathrm{i}}$ WISEA J105536.09-575042.1: Using the 2MASS-to-WISE time baseline we obtain a motion, $\left(\mu_{\alpha}, \mu_{\delta}\right)$, of $(-196.5 \pm 9.3,128.2 \pm 9.3) \mathrm{mas}^{-1}$ for this object. The other component, UPM J1056-5750, has a published motion of $(-194.4 \pm 7.4,121.9 \pm 6.9)$ mas $^{-1}{ }^{-1}$ (Finch et al. 2010). These motions are identical within their $1 \sigma$ errors, so this is very likely a common-proper-motion system.

${ }^{\mathrm{j}}$ WISEA J113333.67-414016.7: Using the 2MASS-to-WISE time baseline we obtain a motion, $\left(\mu_{\alpha}, \mu_{\delta}\right)$, of $(-152.9 \pm 9.2,-120.1 \pm 8.5)$ mas $^{-1}{ }^{-1}$ for this object. The other component, $\mathrm{CD}-406796$, has a published motion of $(-169.7 \pm 1.9,-117.3 \pm 1.7) \mathrm{mas} \mathrm{yr}^{-1}(\mathrm{H} \varnothing \mathrm{g}$ et al. 2000). These motions are identical within their $2 \sigma$ errors, so this is likely a common-proper-motion system.

${ }^{\mathrm{k}}$ WISEA J121058.26-461204.5: Using the 2MASS-to-WISE time baseline we obtain a motion, $\left(\mu_{\alpha}, \mu_{\delta}\right)$, of $(101.3 \pm 8.3,167.7 \pm 8.1)$ mas yr $^{-1}$ for this object.

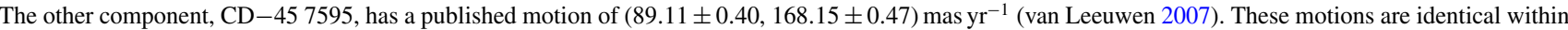
their $2 \sigma$ errors, so this is likely a common-proper-motion system.

${ }^{1}$ WISEA J124014.80+204752.7: Using the 2MASS-to-WISE time baseline we obtain a motion, $\left(\mu_{\alpha}, \mu_{\delta}\right)$, of $(205.6 \pm 10.6,-369.1 \pm 10.8) \mathrm{mas}^{-1} \mathrm{for}^{-1}$ this object. The other component, BD+21 2442, has a published motion of $(202.04 \pm 1.44,-368.45 \pm 0.99)$ mas $_{\mathrm{yr}^{-1}}$ (van Leeuwen 2007). These motions are identical within their $1 \sigma$ errors, so this is very likely a common-proper-motion system.

${ }^{\mathrm{m}}$ WISEA J124726.75-434441.8: Using the 2MASS-to-WISE time baseline we obtain a motion, $\left(\mu_{\alpha}, \mu_{\delta}\right)$, of $(-182.0 \pm 7.1,-95.4 \pm 7.1) \mathrm{mas}^{-1} \mathrm{for}^{-1}$ is object. The other component, CD-43 7881, has a published motion of $(-196.60 \pm 0.80,-94.31 \pm 0.89)$ mas $_{\mathrm{yr}^{-1}}$ (van Leeuwen 2007). These motions are identical within their $2 \sigma$ errors, so this is likely a common-proper-motion system.

${ }^{\mathrm{n}}$ WISEA J125435.54-381111.7: Using the 2MASS-to-WISE time baseline we obtain a motion, $\left(\mu_{\alpha}, \mu_{\delta}\right)$, of $(161.1 \pm 7.9,-25.4 \pm 7.8)$ mas yr ${ }^{-1}$ for this object. The other component, TYC 7772-1225-1, has a published motion of $(149.6 \pm 2.8,-40.1 \pm 2.7)$ mas $_{\mathrm{yr}^{-1}}$ (Høg et al. 2000). These motions are identical to within $2 \sigma$, so this could be a common-proper-motion binary. In the UCAC4 Catalog this pair has listed motions of $(147.6 \pm 0.7,-38.2 \pm 1.0)$ mas $\mathrm{yr}^{-1}$ for the brighter component and $(144.5 \pm 0.8,-29.0 \pm 0.8) \mathrm{mas} \mathrm{yr}^{-1}$ for the fainter component. Although the $10 \sigma$ discrepancy in Decl. would seem to rule these out as a cpm pair, UCAC4 measurements can be unreliable and thus we still list this pair as a possible physical system.

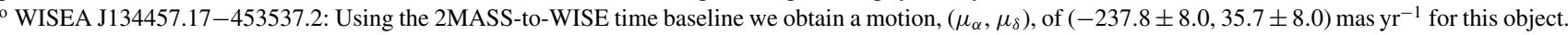
The other component, LTT 5330, has a published motion of $(-240.51 \pm 13.1,25.99 \pm 13.1)$ mas yr$^{-1}$ (Röser et al. 2008). These motions are identical within their $1 \sigma$ errors, so this is very likely a common-proper-motion system.

${ }^{\mathrm{p}}$ WISEA J140350.20-592348.0: Using the 2MASS-to-WISE time baseline we obtain a motion, $\left(\mu_{\alpha}, \mu_{\delta}\right)$, of $(30.5 \pm 8.7,-494.3 \pm 8.5)$ mas yr $^{-1}$ for this object.

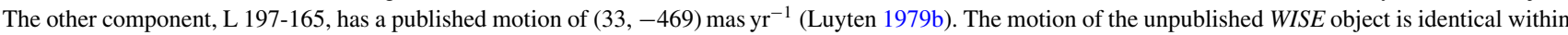
its $1 \sigma$ errors to the R.A. motion of the Luyten object but discrepant by $\sim 3 \sigma$ from the Luyten Decl. measurement. Given the large motion in Decl. and the proximity of the sources, this is still likely a common-proper-motion system.

${ }^{\mathrm{q}}$ WISEA J140924.56-305534.1: Using the 2MASS-to-WISE time baseline we obtain a motion, $\left(\mu_{\alpha}, \mu_{\delta}\right)$, of $(-456.4 \pm 8.1,-213.8 \pm 8.0)$ mas ${ }^{-1}$ for this object. The other component, LHS 2807, has a published motion of $(-460.60 \pm 3.68,-219.82 \pm 3.02)$ mas $_{\mathrm{yr}^{-1}}$ (van Leeuwen 2007). These motions are identical to $<1 \sigma$, so this is likely a common-proper-motion system.

${ }^{\mathrm{r}}$ WISEA J142053.60-050137.8: Using the 2MASS-to-WISE time baseline we obtain a motion, $\left(\mu_{\alpha}, \mu_{\delta}\right)$, of $(-131.2 \pm 10.2,-55.4 \pm 10.0)$ mas $\mathrm{yr}^{-1}$ for this object. The other component, BD-04 3668, has a published motion of $(-137.5 \pm 3.5,-58.0 \pm 3.2)$ mas $_{\mathrm{yr}^{-1}}$ (Roeser \& Bastian 1988). These motions are identical to $<1 \sigma$, so this is likely a common-proper-motion system.

${ }^{\mathrm{s}}$ WISEA J171828.99-224630.2 and WISEA J171826.98-224543.5: Using the 2MASS-to-WISE time baseline we obtain a motion, $\left(\mu_{\alpha}, \mu_{\delta}\right)$, of $(-149.3 \pm 8.9$,

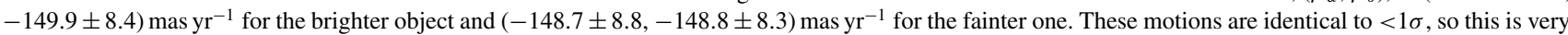
likely a common-proper-motion pair.

${ }^{t}$ WISEA J172230.07-695119.2 and WISEA J172237.14-695112.2: Using the 2MASS-to-WISE time baseline we obtain a motion, $\left(\mu_{\alpha}, \mu_{\delta}\right)$, of $(-145.6 \pm 10.4$,

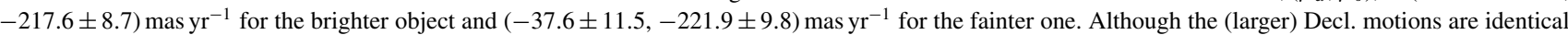
to $<1 \sigma$, the R.A. motions are discrepant by $>9 \sigma$. A visual check of the system more strongly supports common proper motion than do the actual measurements. We suspect that the WISE astrometry for the fainter source in the pair may be corrupted by a background object—seen at nearly equal magnitude in the earlier DSS2 images-that lies near its position in the WISE data.

" WISEA J201605.71-115838.5 and WISEA J201605.96-115916.7: Both of these objects are listed in the UCAC4 Catalog, but no other literature is available. Using the 2MASS-to-WISE time baseline we obtain motions, $\left(\mu_{\alpha}, \mu_{\delta}\right)$, of $(-72.4 \pm 8.9,-141.1 \pm 7.5) \mathrm{mas}^{-1} \mathrm{yr}^{-1}$ for the brighter component and $(-69.4 \pm 9.0$, $-134.4 \pm 7.7) \mathrm{mas} \mathrm{yr}^{-1}$ for the dimmer component, which are identical within their $1 \sigma$ errors. In the UCAC4 Catalog this pair has listed motions of $(-70.9 \pm 2.1$,

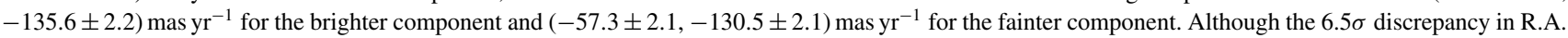
would seem to rule these out as a cpm pair, the secondary appears to be blended with another source of nearly equal magnitude in the DSS2 images. Because visual checks strongly suggest this is a co-moving system, we still list this as a possible physical pair.

${ }^{v}$ WISEA J210630.56-382606.1 and WISEA J210632.73-382640.4: Using the 2MASS-to-WISE time baseline we obtain a motion, $\left(\mu_{\alpha}, \mu_{\delta}\right)$, of $(174.9 \pm 10.1$,

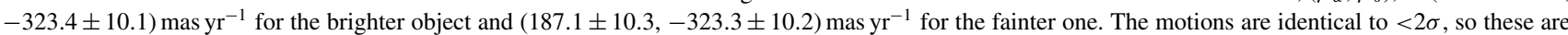
likely a common-proper-motion pair.

${ }^{\mathrm{w}}$ WISEA J214736.41-135335.4: Using the 2MASS-to-WISE time baseline we obtain a motion, $\left(\mu_{\alpha}, \mu_{\delta}\right)$, of $(151.6 \pm 10.0,-300.2 \pm 9.3) \mathrm{mas}^{-1} \mathrm{for} \mathrm{fhis}$ object. The other component, Ross 208, has a published motion of $(152 \pm 5,-296 \pm 5) \mathrm{mas} \mathrm{yr}^{-1}$ (Salim \& Gould 2003). These motions are identical within $<1 \sigma$, so this is very likely a common-proper-motion system.

${ }^{x}$ WISEA J230226.16+801241.8: Using the 2MASS-to-WISE time baseline we obtain a motion, $\left(\mu_{\alpha}, \mu_{\delta}\right)$, of $(-68.1 \pm 9.9,114.1 \pm 9.9)$ mas yr$^{-1}$ for this object. The other component, BD+79 762, has a published motion of $(-53.2 \pm 1.2,127.2 \pm 1.2) \mathrm{mas} \mathrm{yr}^{-1}$ (Høg et al. 2000). These motions are identical within their $2 \sigma$ errors, so this is likely a common-proper-motion system.

${ }^{y}$ This measurement should be treated with caution because the core of this source is heavily saturated in the WISE images. 


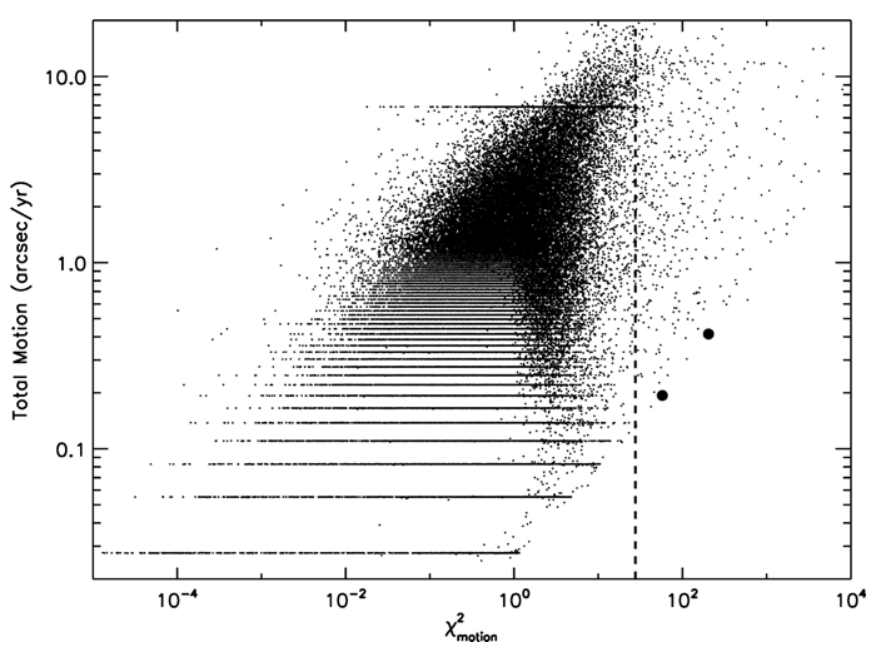

Figure 11. Total motions for sources in AllWISE Atlas Tile 0440p166_ac51 plotted as a function of $\chi^{2}$ motion. Objects to the right of the dashed line have $\chi_{\text {motion }}^{2}>27.63$ and are considered to have statistically significant motions. The previously known motion stars LTT 10950 and NLTT 9223 are shown by the larger dots. The quantized motion measures (further illustrated in the upper panel of Figure 13; see Section 3.5.1) are seen for small motion values in the lower portion of the plot, and the motion "pile-up" for spurious sources (further illustrated in the lower panel of Figure 13; see Section 3.5.2) is seen near the top of the plot at a value of $6 . .875 \mathrm{yr}^{-1}$.

characterized by the Gaussian uncertainties). This limit on $Q$ corresponds to $\chi^{2}$ motion $>27.63$. Sources in this tile that satisfy this criterion are found to the right of the dashed line in Figure 11. Although a few of these objects are motion stars (see Section 3.4.2), other sources have complications that create high values of $\chi^{2}$ motion but do not have real motions. Examples are contaminated and spurious objects flagged as such by $c c_{-}$flags, objects with low signal-to-noise in all bands, and blended or extended sources that can often be identified by the $n b, w$ ?chi2, and rchi 2 criteria. ${ }^{20}$ Other examples of false motion sources are given in Section 3.4.3.

\subsubsection{Real Moving Sources}

A total of 16 objects in Figure 11 passed additional criteria-no blending $(n b=1), w$ ? rchi2 values indicating a point source ( $<2.0$ in all bands), and other criteria as further explained in Section 4.1. Only two of these, highlighted by larger dots in the figure, also passed a visual inspection step showing them to be point-like, unconfused, and moving (if present in data from earlier surveys). Both are previously published motion stars. The first, WISEA J025517.56+161832.7, has AllWISE measured motions in (R.A., Decl.) of (367 $\pm 29,190 \pm$ 29) mas $\mathrm{yr}^{-1}$ and is the known motion star LTT 10950 with published proper motions in (R.A., Decl.) of (203.09 \pm 0.82 , $-47.76 \pm 0.58)$ mas $\mathrm{yr}^{-1}$ (van Leeuwen 2007). The discrepancy in measured values is likely due more to the fact that this source is heavily saturated in AllWISE ( $W 1=5.7 \mathrm{mag}$ ) rather than to parallax, which is only $37.32 \pm 0.66$ mas (van Leeuwen 2007). The second source, WISEA J025326.12+172429.8, has AllWISE measured motions in (R.A., Decl.) of $(-55 \pm 26$, $-185 \pm 25)$ mas $\mathrm{yr}^{-1}$ and is the known motion star NLTT 9223 with published proper motions in (R.A., Decl.) of $(20,-258)$ mas $\mathrm{yr}^{-1}$ (Lépine \& Shara 2005). This source is also saturated in AllWISE ( $W 1=7.7 \mathrm{mag}$ ), which is likely the

20 Throughout the paper, we use the shorthand " $w$ ?chi2" to refer to $w 1$ chi2, w2 chi2, w3chi2, and $w 4$ chi2. reason for the poor agreement between AllWISE and published values.

This Tile highlights the typical success rate in finding new motion objects. Generally fewer than two dozen motion candidates are retained by the automated criteria, and only a small number survive the visual inspection step. Most of those that remain have been previously identified as motion objects by other surveys. Given that we identified 3525 new motion objects over the entire sky and that there are 18,240 Atlas Tiles total, the average Tile does not contain a new discovery.

\subsubsection{False Moving Sources}

False-motion sources generally fall into one of the following categories. Representative examples and their probable causes are discussed below.

1. Blended/extended sources: As in any motion survey, blended or extended objects in AllWISE create the potential for false motions. One such source is WISEA J130716.06+061032.7, an object whose AllWISE measurements indicate a significant motion of $4795 \pm 471$ and $2381 \pm 493$ mas $^{-1}{ }^{-1}$ in R.A. and Decl., respectively. A very faint, extended source is seen at this position in the Sloan Digital Sky Survey (SDSS) $r$ and $i$ band imagery taken in 2003 (Figure 12(a)), so the source is clearly not moving at the rates indicated by AllWISE. This source is extended in the $W 2$ band and the photocenters at $W 1$ and $W 2$ do not align, likely giving rise to the false motion.

2. Spurious small-separation, same-tile (SSST) sources: ${ }^{21}$ WISEAR J103558.13-370522.0 is a source in the AllWISE Reject Table with a seemingly high and statistically significant motion; AllWISE measures $3527 \pm 350$

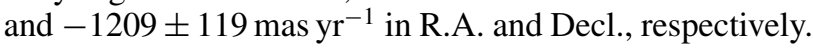
This, however, is a spurious small-separation, same-tile (SSST) source and is flagged as such by the rel flag in the AllWISE Reject Table. As seen in Figure 12(b), this source falls in the wings of a real object, named WISEA J103558.04-370520.9, that is found in the AllWISE Source Catalog and that has motion measures of $-4 \pm 44$ and $27 \pm 44$ mas $\mathrm{yr}^{-1}$ in R.A. and Decl., respectively, indicating no apparent motion. Users are cautioned to pay particular attention to the rel flag when using motion measures from the AllWISE Reject Table.

3. Flux transients: Flux variables can sometimes trigger false motions. When an object appears at only one epoch, a nearby noise blip at the other epoch(s) may trigger a false, generally large motion. Because the derived motion is so large, it may appear to be statistically significant despite having rather large errors. An example of this is WISEA

\footnotetext{
21 An SSST source is part of a group of detections with unphysically small separations extracted on the same Atlas Tile and having nearly identical positions and fluxes. The false sources in these groups are created by a coding error in the AllWISE processing pipeline. The photometry routine works on detections in descending order of brightness; the intention was to process the brightest sources first so that their flux could be subtracted from the individual frames before processing fainter sources. The software error caused the flux subtraction step to be skipped in most cases. For a faint source in the wings of a brighter source, this error can cause the faint source to migrate to the flux from the brighter object because that minimizes $\chi^{2}$ better than a near zero-motion solution near the faint-source detection position itself. Post-processing identified sources in these SSST groups, and the rel parameter was used to flag these in the Catalog and Reject Table. Sources thought to be the original, correct entries have rel codes of $s$ or $c$ and were considered for inclusion in the AllWISE Source Catalog; sources believed to be unreliable members of the SSST group were given $r e l=r$ and will be found only in the AllWISE Reject Table. Sources with $\mathrm{rel}=$ null are not affected by the SSST phenomenon.
} 


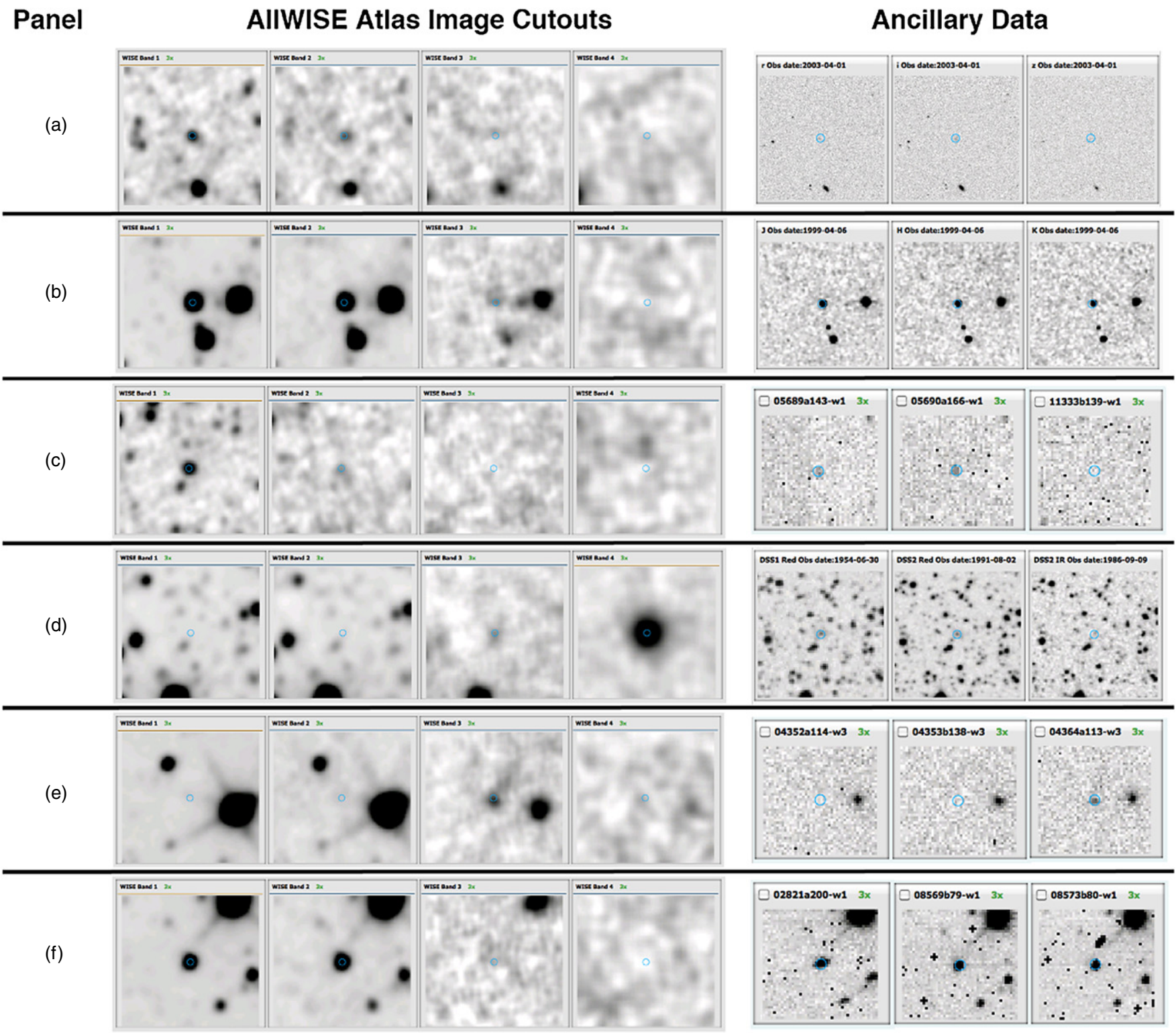

Figure 12. Seven categories of false-motion sources. The $W 1, W 2, W 3$, and $W 4$ AllWISE Atlas Image fields $\left(2^{\prime} \times 2^{\prime}\right.$ with north up and east to the left) along with three ancillary images are shown for each example. The faint blue circle denotes the position of the AllWISE source. Ancillary images are identified as-(a) SDSS $r$, $i$, and $z$. Note that a faint source is seen at the AllWISE position. (b) 2MASS $J, H$, and $K_{s}$. Note that the blue circle is not well centered on the bright source in either the AllWISE or 2MASS images. (c) Representative WISE Level $1 \mathrm{~b}$ frames at $W 1$; the first two frames are from the first epoch of WISE observations and the third frame is from the second epoch. Note that the object is seen only at the first epoch. (d) DSS1 $R$ and DSS2 $R$ and $I$. Note that there is a faint source at the AllWISE position. (e) Three WISE Level $1 \mathrm{~b}$ frames at W3. Note that the first two show no source at the location of the AllWISE object; the third is the only Level $1 \mathrm{~b}$ image at this sky location showing a source at this position. (f) The only first-epoch WISE Level $1 \mathrm{~b}$ image at $W 1$ along with two representative second-epoch $W 1$ images. Note that the first-epoch frame is significantly smeared due to spacecraft motion.

(A color version of this figure is available in the online journal.)

J122559.53+070005.2, which has AllWISE-reported motions of $24902 \pm 4071$ and $11567 \pm 7922 \mathrm{mas} \mathrm{yr}^{-1}$ in R.A. and Decl., respectively. This object appears only in the first epoch and not the second (Figure 12(c)), as confirmed by the AllWISE Multi-Epoch Source Table, which gives a mean $w 1$ mpro value of $\sim 15.3 \mathrm{mag}$ for the first epoch and limits of $>17.0$ mag for the second.

4. W3- and W4-dominated sources: Sources that are detected only at $W 3$ and/or $W 4$ may show spurious motions. Such sources will be seen only at the earlier epochs before cryogen was exhausted and not at the later epochs when those bands were not operational. Nearby noise blips at the later epochs in $W 1$ and/or $W 2$ can trigger false motions.
One such example is the planetary nebula PN SB 24, also known as WISEA J185716.62-175050.4 (Figure 12(d)), which has AllWISE-measured motions of $6243 \pm 1101$ and $-11981 \pm 1162$ mas $^{-1} r^{-1}$ in R.A. and Decl., respectively, despite the fact that the nebula is known to have near-zero proper motion (Kerber et al. 2008).

5. Cosmic rays in low-coverage areas: Areas having less than five frames of coverage do not reap the benefit of outlier rejection $^{22}$ in the coaddition step, so spurious sources can bleed through into the coadds. In some cases, these

${ }^{22}$ See http://wise2.ipac.caltech.edu/docs/release/allsky/expsup/sec4_4f.html\# outrej in the WISE All-Sky Release Explanatory Supplement. 
spurious sources have falsely measured, yet apparently significant, motions in AllWISE. One example is WISEA J210220.65-083948.6 (Figure 12(e)), a cosmic ray that appears in only one $W 3$ frame (04364a113) in an area of sky where that $W 3$ frame is the only one of acceptable quality for AllWISE processing. A nearby noise blip in another band at a different epoch nonetheless causes AllWISE to measure a large, although false, motion for it of $4573 \pm 1094$ and $6531 \pm 1213$ mas yr$^{-1}$ in R.A. and Decl., respectively.

6. Sources falling in a streaked frame in a low-coverage area: Another consequence of low-coverage areas is the increased influence of the occasional poorer quality frame going into the coadd. The most extreme example is one in which only a single frameset is available for one of the WISE epochs, and that frameset has non-optimal image quality. False motions may result, as is the case for source WISEA J061658.37+701209.5 (Figure 12(f)). This object lies in an area of sky with single-frameset coverage (02821a200) at the first epoch, but this frameset is slightly smeared due to momentum dumping ${ }^{23}$ by the spacecraft. The astrometric solution for the entire frameset is slightly biased in R.A. because of this smearing. Data at the later epoch are not affected. As a result of the mismatch in positions across epochs, a false motion of $1547 \pm 129$

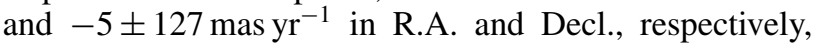
is created. It should be noted that this is an extremely rare occurrence in AllWISE, as Quality Assurance flagged almost all of the smeared frames and eliminated them from consideration for coaddition. Nonetheless, users are advised to treat with caution any motion measures in areas of lowcoverage at a single epoch.

\subsection{Other Caveats}

\subsubsection{Motion Measurements are Quantized}

A plot of AllWISE motion measurements in a single Atlas Tile (upper panel of Figure 13) shows that certain, discrete values have a higher frequency of occurrence. The solution for motion values and source positions is an iterative process whereby a minimization of $\chi^{2}$ is sought in the phase space of source position and motion. Convergence is rapidly achieved for position estimates, using initial values. On the other hand, convergence on motion values is achieved only after one or more iterative steps. The step size used for motion estimates is $27.5 \mathrm{mas} \mathrm{yr}^{-1}$, and converged motion values are thus quantized at multiples of this value. ${ }^{24}$

\subsubsection{Spurious Motion Measurements Show "Pile-up" at Large Values}

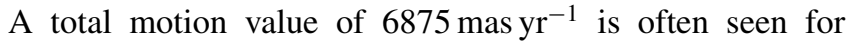
spurious sources in AllWISE. The "pile-up" of motion measures at this value is illustrated in the lower panel of Figure 13. The algorithm for minimization of $\chi^{2}$ has a maximum number of iterations of 250. Each iteration step for motion uses, as stated

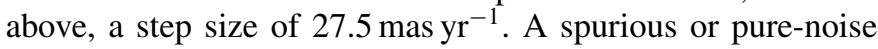
solution that has not converged after the maximum number

\footnotetext{
23 See http://wise2.ipac.caltech.edu/docs/release/allwise/expsup/sec4_2.html\# torque in the AllWISE Explanatory Supplement.

24 See http://wise2.ipac.caltech.edu/docs/release/allwise/expsup/sec5_3bii. html\#gradient-descent_algo in the AllWISE Explanatory Supplement for further information.
}
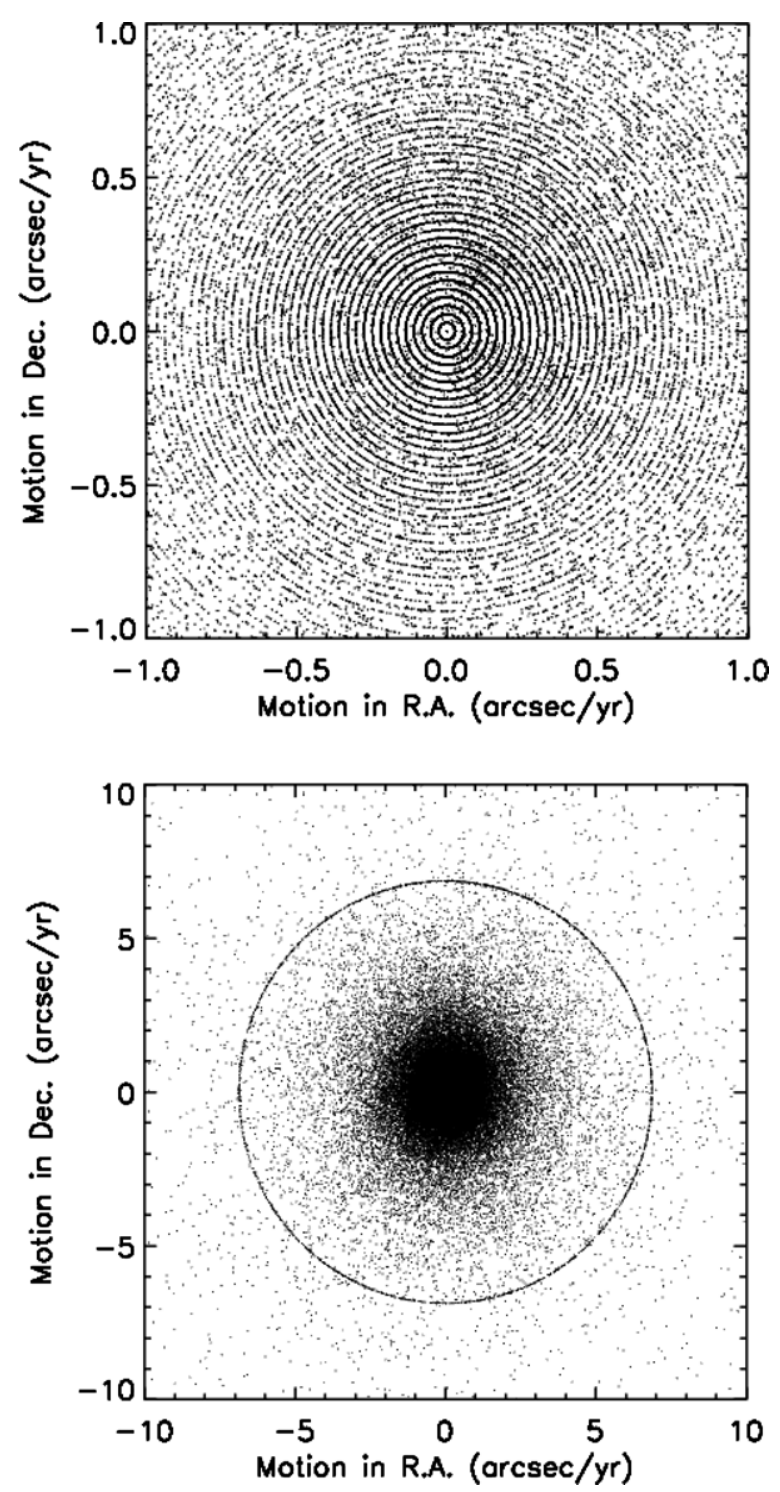

Figure 13. Plots of the AllWISE-measured motions in R.A. plotted against the AllWISE-measured motions in Decl. for a typical Atlas Tile (1174p075_ac51). (Upper panel) A zoom-in showing motion quantization at small values. (Lower panel) A zoom-out showing the motion "pile-up" at a value of $6.875 \mathrm{yr}^{-1}$.

of steps will lead to a motion at the "pile-up" value ${ }^{25}$ of

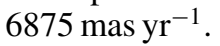

\section{A CATALOG OF MOTION DISCOVERIES}

\subsection{Criteria for Selecting Motion Candidates}

As part of AllWISE pipeline processing at IPAC, the Quality Assurance code filtered the list of detections from each Atlas Tile to identify objects believed to be moving significantly. These criteria were used to select only the choicest motion objects from the source lists and to keep the number of candidates manageable on the tight schedule allotted for AllWISE Quality Assurance checks. The criteria used are as follows.

1. To ensure that the object is not flagged as an artifact, the cc_flags parameter was required not to have a capital-letter

\footnotetext{
25 See http://wise2.ipac.caltech.edu/docs/release/allwise/expsup/sec5_3bii. html\#effect_noise_motion_solution in the AllWISE Explanatory Supplement for more details.
} 
value at any position in its four-character code, in which the four characters refer to the four WISE bands. Capital letters are used when the software has determined in that band that the object was a spurious detection of either a diffraction ("D") spike, a scattered light halo ("H") surrounding a bright star, an optical ("O") ghost image, or persistence ("P")-i.e., a short-term latent image-left behind by a bright source on the detector. Values of $c c_{\text {fflags having }}$ only lower-case versions of these same letters (indicating that the object is likely real and only contaminated by an artifact) or zero (indicating that the source is unaffected by known artifacts) were allowed.

2. To ensure that the object is point-like, a value of $n b=1$ (no blends) was required. Also, the motion-fit, reduced $\chi^{2}$ values in all bands, $w$ ?rchi2_pm, were required to be less than two to eliminate sources that are obviously extended.

3 . To eliminate more poorly measured sources from consideration, the signal-to-noise ratio, $w$ ?snr, was required to be greater than seven in at least one band.

4. To ensure that the code selected only those objects with robust and statistically significant motions, the following criteria were applied: (a) The ratio of the reduced $\chi^{2}$ value between the stationary and motion fit, rchi2/rchi2 $2_{-}$m, was required to be greater than 1.03. This ensures that the motion-fit solution significantly improves the overall reduced $\chi^{2}$ value compared to the stationary fit. (b) As explained in Section 3.4 above, the $\chi^{2}$ motion criterion was required to be greater than 27.63 to select only those objects with statistically significant motions. (c) The second and third characters of pmcode were required to be " $\mathrm{N}$ " and " 0 ," respectively. A value of "N" means that no blendswapping has occurred and a value of " 0 " means that the offset between the stationary-fit and the motion-fit position at the mean epoch is less than $1^{\prime \prime}$. These values were chosen because blended sources often have blend swapping, and spurious sources often have unphysically large positional differences at the mean epoch between the non-motion and motion solutions. ${ }^{26}$

5. To ensure that the motion measurement has a solid foundation of $W 1$ - and W2-band data underlying it, we experimented with various values of $w 1 m, w 2 m$, and w1mJDmax - w1mJDmin. The first two parameters specify the number of frames in $W 1$ and $W 2$, and the third parameter specifies the time difference between the earliest and latest frames in $W 1$. In the end, these parameters were all set to be greater than zero because regions of very sparse coverage were found not to contribute a large number of spurious motion sources, mainly because the vast majority of the sky is well covered across two or more epochs.

These criteria were written to eliminate blended/extended sources, spurious objects, and pure noise. Other contaminants listed in Section 3.4.3- the SSST sources, flux transients, W3and $W 4$-dominated sources, and cosmic rays and streaked frames in low coverage areas-were discovered as a result of these Quality Assurance checks. These contaminants were generally easy to recognize via a visual inspection step, which displayed images of the field in the AllWISE $W 1, W 2, W 3$, and W4 coadds along with Two Micron All Sky Survey (2MASS) images at $J, H$, and $K_{s}$. For WISE objects that were also visible in $2 \mathrm{MASS}$, which represents the vast majority of sources 26 See http://wise2.ipac.caltech.edu/docs/release/allwise/expsup/sec2_1a.
html\#pmcode in the AllWISE Explanatory Supplement. identified, any motion should be obvious in the $10+$ yr between the 2MASS and WISE image sets. ${ }^{27}$ The Quality Assurance scientists checked these images for all candidate motion objects and retained only those objects having no 2MASS source at the AllWISE position.

\subsection{Vetting the List of Candidates}

The Quality Assurance scientists (J.D.K. and S.F.A.) produced a list of 26,850 candidates as a result of the criteria filtering and image checking discussed above. This list was then run (by J.D.K.) through SIMBAD using a $2^{\prime}$ search radius around the AllWISE position to search for previously known objects. If SIMBAD noted a proper motion source within $10^{\prime \prime}$ of the AllWISE position, the object was eliminated from the list. ${ }^{28}$ When known proper motion sources were found outside of $10^{\prime \prime}$, the AllWISE- and SIMBAD-reported motions were checked to see if the object was a very high-motion source, generally $>1^{\prime \prime} \mathrm{yr}^{-1}$. This further check eliminated many known, small-numbered LHS (Luyten Half Second) objects.

The remaining list of objects was then checked (by J.D.K.) against the Fourth US Naval Observatory CCD Astrograph Catalog $^{29}$ (UCAC4; Zacharias et al. 2013) using a $1^{\prime}$ search radius. Objects with significant UCAC4-reported motions were considered to be possible matches to the AllWISE source. The match was considered confirmed if the UCAC4 source appeared to have a motion of the right magnitude and in the correct direction, as inferred from visual inspection of the motion source across the DSS2, 2MASS, and WISE images, to be the AllWISEselected object. Those objects matching a source with reliable motions in UCAC4 were flagged as such but kept in the object list because they have no SIMBAD entries.

After these checks, the list was run again through SIMBAD and further checked against DwarfArchives.org, against a list of published papers (on low-mass objects) likely not to have been ingested into SIMBAD by 2013 December, and against catalogs housed by VizieR. These checks (by G.N.M. and A.S.) enabled us to eliminate other objects. Objects were kept in the list only when SIMBAD had no reported proper motion information and no links to published papers citing a proper motion value. Thus, a few objects with published spectral types are included in the list because this will be the first refereed publication in SIMBAD giving their motion information. Objects listed only in UCAC4 and other catalogs found in VizieR are noted as such in the final list.

Of the 26,850 motion candidates selected by our Quality Assurance criteria, we found that 18,862 objects (70\%) were already published and had motion information available in SIMBAD. Of the remainder, 3583 objects (13\%) are new discoveries and 4405 objects (16\%) were found to be nonmotion sources after visual inspection of the DSS2 images.

The discovery list of 3583 itself is comprised of two sublists. The first (Table 3 ) contains 3525 objects whose motions could be confirmed using non-WISE data, and the second (Table 4) contains 58 unconfirmed objects lacking counterparts in earlier surveys. For the list of confirmed objects, one of us (A.S.)

\footnotetext{
27 Because the smallest motion measurable with AllWISE is $\sim 0$. $^{\prime} 15 \mathrm{yr}^{-1}$ (Section 3.2.2), this means that objects will have moved $>15^{\prime \prime}$ in the $10+\mathrm{yr}$ between the 2MASS and WISE imaging sets, which is a sufficiently large motion to be obvious by eye.

28 Roughly speaking, a circle of radius $10^{\prime \prime}$ around the AllWISE J2000 position at epoch 2010.54 will capture all SIMBAD motion objects, reported using their J2000 positions and epoch 2000.00, moving less than $\sim 1^{\prime \prime} \mathrm{yr}^{-1}$. 29 Available at http://irsa.ipac.caltech.edu.
} 
Table 3

\begin{tabular}{|c|c|c|c|c|c|c|c|c|c|c|c|}
\hline WISEA Designation & $\begin{array}{c}\text { 2MASS } J \\
\text { (mag) } \\
(2)\end{array}$ & $\begin{array}{c}\text { 2MASS } H \\
\text { (mag) } \\
\text { (3) }\end{array}$ & $\begin{array}{c}\text { 2MASS } K_{S} \\
\text { (mag) } \\
\text { (4) }\end{array}$ & $\begin{array}{c}\begin{array}{c}W 1 \\
(\mathrm{mag})\end{array} \\
(5)\end{array}$ & $\begin{array}{c}\begin{array}{c}W 2 \\
\text { (mag) }\end{array} \\
(6)\end{array}$ & $\begin{array}{c}\text { AllWISE } \\
\text { R.A. Motion } \\
\left(\mathrm{mas} \mathrm{yr}^{-1}\right) \\
(7)\end{array}$ & $\begin{array}{c}\text { AllWISE } \\
\text { Decl. Motion } \\
\left(\mathrm{mas} \mathrm{yr}^{-1}\right) \\
(8)\end{array}$ & $\begin{array}{c}\text { Computed } \\
\mu_{\alpha}^{\mathrm{a}} \\
\left(\mathrm{mas} \mathrm{yr}^{-1}\right) \\
(9)\end{array}$ & $\begin{array}{c}\text { Computed } \\
\mu_{\delta}^{\mathrm{a}} \\
\left(\mathrm{mas} \mathrm{yr}^{-1}\right) \\
(10)\end{array}$ & Flag $^{\mathrm{b}}$ & Note $^{\mathrm{c}}$ \\
\hline J000136.86-010146.9 & $12.363 \pm 0.021$ & $11.831 \pm 0.020$ & $11.565 \pm 0.021$ & $11.374 \pm 0.022$ & $11.202 \pm 0.021$ & $-166 \pm 49$ & $-378 \pm 48$ & $-38.5 \pm 11.4$ & $-233.1 \pm 10.5$ & 0 & 0 \\
\hline J000138.90-761350.1 & $9.477 \pm 0.021$ & $8.893 \pm 0.024$ & $8.603 \pm 0.023$ & $8.460 \pm 0.023$ & $8.374 \pm 0.020$ & $284 \pm 30$ & $-170=$ & $241.2 \pm 17.1$ & $-16.7 \pm 7.7$ & 0 & 0 \\
\hline J000205.60-322545.9 & $14.079 \pm 0.026$ & $13.472 \pm 0.036$ & $13.216 \pm 0.037$ & $13.085 \pm 0.024$ & $13.023 \pm 0.026$ & $0 \pm 74$ & $0 \pm 73$ & $23.8 \pm 10.1$ & $42.0 \pm 9.8$ & 0 & 0 \\
\hline J000234.41+470030.9 & $10.288 \pm 0.019$ & $9.714 \pm 0.030$ & $9.468 \pm 0.021$ & $9.311 \pm 0.023$ & $9.276 \pm 0.021$ & $-69 \pm 31$ & $-202 \pm 30$ & $-93.4 \pm 9.7$ & $-107.1 \pm 9.5$ & 1 & 0 \\
\hline J000239.96+612015.0 & $12.709 \pm 0.028$ & $12.059 \pm 0.031$ & $11.728 \pm 0.021$ & $11.558 \pm 0.023$ & $11.390 \pm 0.021$ & $201 \pm 28$ & $92 \pm 27$ & $222.8 \pm 9.2$ & $68.5 \pm 9.1$ & 0 & 0 \\
\hline J000416.30-605925.3 & $11.359 \pm 0.021$ & $10.842 \pm 0.022$ & $10.636 \pm 0.021$ & $10.515 \pm 0.022$ & $10.416 \pm 0.020$ & $-258 \pm 36$ & $-218 \pm 35$ & $-228.3 \pm 9.8$ & $-213.8 \pm 9.8$ & 1 & 0 \\
\hline J000533.57+280705.8 & $14.073 \pm 0.022$ & $13.489 \pm 0.033$ & $13.186 \pm 0.031$ & $12.975 \pm 0.024$ & $12.702 \pm 0.026$ & $-355 \pm 65$ & $-346 \pm 66$ & $-205.1 \pm 11.9$ & $-264.8 \pm 10.0$ & 1 & 1 \\
\hline J000622.67-131955.6 & $16.674 \pm 0.125$ & $15.548 \pm 0.105$ & $15.115 \pm 0.126$ & $14.239 \pm 0.027$ & $13.754 \pm 0.042$ & $-416 \pm 123$ & $-852 \pm 128$ & $-239.2 \pm 17.3$ & $-413.2 \pm 16.3$ & 2 & 0 \\
\hline J000915.71-285019.7 & $14.744 \pm 0.035$ & $14.143 \pm 0.042$ & $13.800 \pm 0.045$ & $13.624 \pm 0.026$ & $13.435 \pm 0.033$ & $44 \pm 101$ & $-229 \pm 103$ & $86.2 \pm 11.2$ & $32.0 \pm 10.9$ & 0 & 0 \\
\hline J001102.05-421417.7 & $10.903 \pm 0.021$ & $10.37 \pm 0.026$ & $10.094 \pm 0.021$ & $9.912 \pm 0.022$ & $9.774 \pm 0.019$ & $-399 \pm 37$ & $-190 \pm 36$ & $-248.9 \pm 11.1$ & $-40.6 \pm 9.4$ & 1 & 0 \\
\hline
\end{tabular}

Notes.

a This is the motion measured between the 2MASS and WISE epochs.

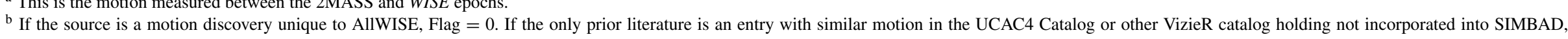

Flag $=1$. If the object appears only in the Luhman (2014) list, Flag $=2$. If the object appears both in the Luhman (2014) list and in a prior catalog not incorporated into SIMBAD, Flag $=3$.

${ }^{c}$ If there is an additional note about this source at the end of the table, Note $=1$.

(This table is available in its entirety in a machine-readable form in the online journal. A portion is shown here for guidance regarding its form and content.) 
Table 4

AllWISE Motion Candidates Lacking 2MASS Counterparts ${ }^{\mathrm{a}}$

\begin{tabular}{|c|c|c|c|c|c|c|}
\hline WISEA Designation & $\begin{array}{c}\begin{array}{c}W 1 \\
(\mathrm{mag})\end{array} \\
(2)\end{array}$ & $\begin{array}{c}\begin{array}{c}W 2 \\
(\mathrm{mag})\end{array} \\
(3)\end{array}$ & $\begin{array}{c}\text { AllWISE } \\
\text { R.A. Motion } \\
\left(\mathrm{mas} \mathrm{yr}^{-1}\right) \\
(4)\end{array}$ & $\begin{array}{c}\text { AllWISE } \\
\text { Decl. Motion } \\
\left(\mathrm{mas} \mathrm{yr}^{-1}\right) \\
(5)\end{array}$ & Flag $^{\mathrm{b}}$ & Note $^{\mathrm{c}}$ \\
\hline J001152.64-202826.4 & $16.443 \pm 0.072$ & $15.261 \pm 0.093$ & $-8857 \pm 686$ & $16150 \pm 720$ & 0 & 0 \\
\hline J001449.42-055522.7 & $16.313 \pm 0.073$ & $16.217 \pm 0.233$ & $5931 \pm 929$ & $8629 \pm 936$ & 1 & 0 \\
\hline J003314.92-465936.0 & $16.094 \pm 0.051$ & $15.612 \pm 0.095$ & $-8412 \pm 436$ & $4870 \pm 442$ & 1 & 0 \\
\hline J003647.68-122155.3 & $15.863 \pm 0.054$ & $15.522 \pm 0.124$ & $-6302 \pm 619$ & $-1310 \pm 572$ & 1 & 0 \\
\hline J005429.43-595619.9 & $16.237 \pm 0.057$ & $15.772 \pm 0.118$ & $-2302 \pm 570$ & $4102 \pm 547$ & 0 & 0 \\
\hline J005519.24-491935.3 & $16.196 \pm 0.057$ & $16.069 \pm 0.153$ & $5645 \pm 552$ & $2333 \pm 557$ & 1 & 0 \\
\hline J005853.15-555722.4 & $15.540 \pm 0.038$ & $15.628 \pm 0.099$ & $5248 \pm 349$ & $826 \pm 327$ & 1 & 0 \\
\hline J010133.97-551653.9 & $17.206 \pm 0.122$ & $15.587 \pm 0.099$ & $-8224 \pm 861$ & $9173 \pm 886$ & 0 & 0 \\
\hline J010213.98-344757.7 & $16.059 \pm 0.050$ & $15.145 \pm 0.069$ & $1864 \pm 454$ & $5155 \pm 486$ & 0 & 0 \\
\hline J010309.96-151101.0 & $15.484 \pm 0.042$ & $15.500 \pm 0.109$ & $-4098 \pm 363$ & $1027 \pm 384$ & 1 & 0 \\
\hline J011133.57-412722.5 & $16.359 \pm 0.058$ & $16.203 \pm 0.148$ & $-19976 \pm 722$ & $13761 \pm 761$ & 1 & 0 \\
\hline J011759.72-281749.6 & $15.494 \pm 0.039$ & $15.399 \pm 0.091$ & $-1752 \pm 381$ & $3048 \pm 369$ & 1 & 0 \\
\hline J012122.26-110214.0 & $16.910 \pm 0.115$ & $15.257 \pm 0.095$ & $-2373 \pm 735$ & $5624 \pm 805$ & 0 & 0 \\
\hline J012255.66-223007.0 & $15.197 \pm 0.035$ & $14.886 \pm 0.067$ & $-1465 \pm 279$ & $226 \pm 281$ & 1 & 0 \\
\hline J014224.00-361822.7 & $16.046 \pm 0.050$ & $15.584 \pm 0.098$ & $-447 \pm 457$ & $-5412 \pm 442$ & 1 & 0 \\
\hline J014446.74-203855.3 & $16.205 \pm 0.063$ & $15.607 \pm 0.128$ & $6287 \pm 626$ & $2423 \pm 661$ & 1 & 0 \\
\hline J014507.92-355631.8 & $16.139 \pm 0.053$ & $15.915 \pm 0.123$ & $3276 \pm 485$ & $-633 \pm 492$ & 1 & 0 \\
\hline J014804.04-712828.3 & $16.360 \pm 0.051$ & $15.072 \pm 0.053$ & $-1697 \pm 428$ & $-3448 \pm 394$ & 0 & 0 \\
\hline J020801.13-161630.1 & $16.020 \pm 0.055$ & $15.991 \pm 0.152$ & $-3994 \pm 489$ & $11866 \pm 588$ & 1 & 0 \\
\hline J020812.38-275731.9 & $16.617 \pm 0.072$ & $16.350 \pm 0.191$ & $-9498 \pm 602$ & $-185 \pm 616$ & 1 & 0 \\
\hline J024519.98+134918.6 & $16.130 \pm 0.060$ & $15.977 \pm 0.154$ & $3028 \pm 307$ & $1042 \pm 333$ & 1 & 0 \\
\hline J025246.35-262919.1 & $16.385 \pm 0.059$ & $16.346 \pm 0.161$ & $-52 \pm 329$ & $-4234 \pm 340$ & 1 & 0 \\
\hline J031159.56-195310.6 & $15.970 \pm 0.043$ & $15.421 \pm 0.082$ & $-919 \pm 229$ & $5174 \pm 229$ & 0 & 0 \\
\hline J031303.91-453358.2 & $15.359 \pm 0.034$ & $15.389 \pm 0.071$ & $-1661 \pm 152$ & $510 \pm 155$ & 1 & 0 \\
\hline J032307.25-364432.4 & $15.885 \pm 0.042$ & $15.661 \pm 0.094$ & $1023 \pm 196$ & $-3686 \pm 194$ & 1 & 0 \\
\hline J033231.23-245841.9 & $16.376 \pm 0.054$ & $16.078 \pm 0.133$ & $-3320 \pm 288$ & $-2582 \pm 307$ & 1 & 0 \\
\hline J034803.93-354036.0 & $16.058 \pm 0.043$ & $15.580 \pm 0.075$ & $-1593 \pm 196$ & $-4013 \pm 197$ & 0 & 0 \\
\hline J053133.00-304416.7 & $15.447 \pm 0.038$ & $15.710 \pm 0.100$ & $-1246 \pm 335$ & $-2708 \pm 340$ & 1 & 0 \\
\hline J053509.97-313855.9 & $16.344 \pm 0.061$ & $16.244 \pm 0.181$ & $2027 \pm 749$ & $-4774 \pm 788$ & 1 & 0 \\
\hline J054631.32-311749.1 & $15.921 \pm 0.048$ & $15.706 \pm 0.108$ & $-1959 \pm 556$ & $3282 \pm 619$ & 1 & 0 \\
\hline J055034.35-363805.8 & $15.806 \pm 0.044$ & $15.291 \pm 0.076$ & $-3399 \pm 394$ & $-2114 \pm 430$ & 0 & 0 \\
\hline J055202.16-534725.1 & $16.253 \pm 0.043$ & $16.068 \pm 0.095$ & $3637 \pm 433$ & $1876 \pm 467$ & 1 & 0 \\
\hline J055537.68-175339.9 & $16.019 \pm 0.053$ & $15.611 \pm 0.107$ & $3813 \pm 545$ & $2242 \pm 596$ & 1 & 0 \\
\hline J055658.63-140702.8 & $15.367 \pm 0.040$ & $15.230 \pm 0.078$ & $-914 \pm 344$ & $-2203 \pm 364$ & 1 & 0 \\
\hline J065959.23+780829.9 & $15.600 \pm 0.039$ & $15.223 \pm 0.068$ & $-782 \pm 330$ & $-2872 \pm 367$ & 0 & 0 \\
\hline J085510.74-071442.5 & $16.231 \pm 0.064$ & $13.704 \pm 0.033$ & $-4188 \pm 267$ & $226 \pm 283$ & 2 & 1 \\
\hline $\mathrm{J} 105042.60+140202.3$ & $17.124 \pm 0.138$ & $15.568 \pm 0.124$ & $-1521 \pm 800$ & $-13560 \pm 912$ & 0 & 0 \\
\hline J111202.32+324123.7 & $17.128 \pm 0.112$ & $16.658 \pm 0.268$ & $5648 \pm 591$ & $5618 \pm 630$ & 0 & 0 \\
\hline J113654.49-201658.7 & $15.766 \pm 0.046$ & $15.718 \pm 0.159$ & $3239 \pm 407$ & $-4756 \pm 441$ & 1 & 0 \\
\hline J121607.98+191003.1 & $15.926 \pm 0.055$ & $15.313 \pm 0.103$ & $6708 \pm 519$ & $-4289 \pm 562$ & 1 & 0 \\
\hline J121903.27-084702.2 & $16.200 \pm 0.067$ & $16.594 \pm 0.283$ & $-3987 \pm 637$ & $-1822 \pm 703$ & 1 & 0 \\
\hline J125728.42+464457.6 & $16.574 \pm 0.075$ & $15.911 \pm 0.135$ & $6348 \pm 519$ & $-2077 \pm 543$ & 1 & 0 \\
\hline J130825.50+331256.1 & $15.736 \pm 0.044$ & $15.265 \pm 0.074$ & $-6619 \pm 288$ & $-2636 \pm 321$ & 1 & 0 \\
\hline J131302.32+030607.4 & $17.254 \pm 0.144$ & $>16.918$ & $-1975 \pm 537$ & $-6980 \pm 618$ & 0 & 0 \\
\hline J134115.18+280218.5 & $17.410 \pm 0.140$ & $>16.837$ & $-3137 \pm 676$ & $10163 \pm 776$ & 0 & 0 \\
\hline J144817.44+490631.2 & $16.053 \pm 0.046$ & $15.364 \pm 0.073$ & $5177 \pm 330$ & $-1900 \pm 357$ & 0 & 0 \\
\hline J151813.45+311515.8 & $17.376 \pm 0.103$ & $16.311 \pm 0.146$ & $7908 \pm 378$ & $-814 \pm 413$ & 0 & 1 \\
\hline J163139.17-244942.7 & $13.245 \pm 0.026$ & $11.004 \pm 0.021$ & $-235 \pm 64$ & $-548 \pm 69$ & 0 & 1 \\
\hline J174254.29+761729.7 & $15.997 \pm 0.036$ & $15.280 \pm 0.047$ & $-2100 \pm 328$ & $-2899 \pm 342$ & 0 & 0 \\
\hline J180512.73+321459.3 & $16.275 \pm 0.057$ & $14.869 \pm 0.051$ & $-2713 \pm 507$ & $3013 \pm 566$ & 0 & 0 \\
\hline $\mathrm{J} 185818.21+804757.5$ & $15.806 \pm 0.036$ & $15.556 \pm 0.066$ & $-1552 \pm 304$ & $-2236 \pm 328$ & 0 & 0 \\
\hline J191837.08+833036.5 & $15.891 \pm 0.038$ & $15.773 \pm 0.078$ & $3143 \pm 359$ & $817 \pm 380$ & 1 & 0 \\
\hline J203712.92-071456.2 & $16.198 \pm 0.064$ & $14.563 \pm 0.056$ & $-1541 \pm 297$ & $2346 \pm 289$ & 0 & 0 \\
\hline J205029.36-344817.4 & $16.044 \pm 0.056$ & $14.761 \pm 0.063$ & $2486 \pm 457$ & $1839 \pm 470$ & 0 & 0 \\
\hline J225750.96-440429.5 & $16.398 \pm 0.055$ & $16.842 \pm 0.257$ & $-11280 \pm 550$ & $11280 \pm 596$ & 1 & 0 \\
\hline J230744.88-200218.5 & $15.295 \pm 0.040$ & $15.455 \pm 0.125$ & $-2117 \pm 365$ & $6388 \pm 375$ & 1 & 0 \\
\hline J232558.27-135406.6 & $16.645 \pm 0.089$ & $16.629 \pm 0.307$ & $-2993 \pm 961$ & $17804 \pm 962$ & 1 & 0 \\
\hline J232822.75-385208.8 & $16.079 \pm 0.057$ & $15.582 \pm 0.111$ & $-1229 \pm 524$ & $-3938 \pm 527$ & 1 & 0 \\
\hline
\end{tabular}

Notes.

${ }^{a}$ Most of the objects in this list are believed not to be actual motion sources. See the text for details.

${ }^{\mathrm{b}}$ If the source is a motion discovery unique to AllWISE, Flag $=0$. If this source is believed to be a flux transient and not a motion object because of its blue $W 1-W 2$ color, Note $=1$. If the object appears in Luhman (2014), Flag $=2$.

${ }^{\mathrm{c}}$ If there is an additional note about this source at the end of the table, Note $=1$.

WISEA J085510.74-071442.5: This object is $28^{\prime \prime}$ from the radio source 3C 209, which has the identifier WISEA J085509.46-071502.9. Although the WISE imaging data show a clear photocentric shift between the two epochs of data, additional imaging is needed to confirm the motion measure. WISEA J151813.45+311515.8: Brightest in W3 band (11.073 \pm $0.097 \mathrm{mag}$ ) but also faintly visible at $W 1$ and $W 2$. WISEA J163139.17-244942.7: May correspond to the embedded, sub-mm source JCMTSE J163138.6-244950 in the $\rho$ Oph star formation complex. 
consulted the 2MASS images to find the WISE source at the 2MASS epoch and then tabulated the 2MASS position and magnitudes from the 2MASS All-Sky Point Source Catalog. These 2MASS associations were then double checked (by J.D.K.) by creating finder charts of DSS, 2MASS and WISE images centered at the position of the 2MASS source. Using these associations, a proper motion was measured for each source using the 2MASS position as the first epoch and the mean WISE All-Sky Source Catalog position as the second epoch.

Because the identification of motion candidates during Quality Assurance took place before selection of objects for the AllWISE Source Catalog (and before the SSST phenomenon was fully recognized), we had to retroactively replace a small number of our motion candidates that appeared only in the AllWISE Reject Table with their more appropriate entry from the AllWISE Source Catalog. In some cases, the entry in the AllWISE Source Catalog does not have a motion significant enough to have been selected by the Quality Assurance criteria. We retain such cases if our independent checks using the 2MASS-to-WISE time baseline confirm that the objects are truly moving. An example of this is WISEA J000205.60-322545.9,

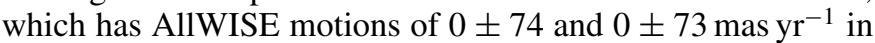
R.A. and Decl., respectively, but whose comparison with the earlier 2MASS position confirms a motion of $23.8 \pm 10.1$ and

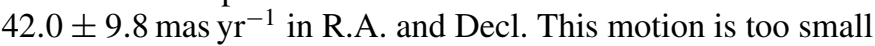
to have been legitimately uncovered by AllWISE, but we list it nonetheless as a serendipitous discovery.

The sky distribution of the confirmed motion objects is shown in Figure 14. The top panel shows the ecliptic projection that is the natural system for the WISE scanning pattern. The threeepoch coverage $\operatorname{areas}^{30}$ at ecliptic longitudes of $\sim 25^{\circ}-48^{\circ}$ and $\sim 200^{\circ}-223^{\circ}$ are, as expected, seen as overdensities. An underdensity in the number of motion objects is seen toward the Galactic Center and along the Galactic mid-plane (bottom panel of Figure 14), as expected in these regions of source confusion and high backgrounds.

The sky distribution of the confirmed objects is compared in Figure 15 to the New Luyten Two Tenths Catalog (NLTT; Luyten $1979 b$ ), a large compilation of motion objects covering the entire sky. As expected, the majority of new discoveries (middle panel of Figure 15) falls primarily in the southern hemisphere because the NLTT is much less complete in those regions (top panel of Figure 15). The clumpiness of these discoveries in the southern hemisphere (e.g., at $11^{\mathrm{h}}<$ R.A. $<22^{\mathrm{h}}$ and Decl. $<-0^{\circ}$ ) is largely a consequence of the WISE three-epoch coverage (middle panel of Figure 14) overlapping areas poorly covered by the NLTT.

\subsection{The Motion Catalog}

The list of all 3525 confirmed AllWISE motion objects is given in Table 3. Column 1 gives the source designation from the AllWISE Source Catalog. Columns 2-4 give the 2MASS $J, H$, and $K_{s}$ magnitudes and associated errors of the source, and Columns 5 and 6 give the AllWISE $W 1$ and $W 2$ profile-fit magnitudes and errors. Columns 7 and 8 give the AllWISEmeasured motions in R.A. and Decl. whereas Columns 9 and 10 give the proper motions computed using the 2MASS-to-WISE time baseline. Column 11 is a flag column indicating whether the

\footnotetext{
30 See the depth-of-coverage sky map (Figure 5) in Section IV.2 of the AllWISE Explanatory Supplement at

http://wise2.ipac.caltech.edu/docs/release/allwise/expsup/sec4_2.html.
}
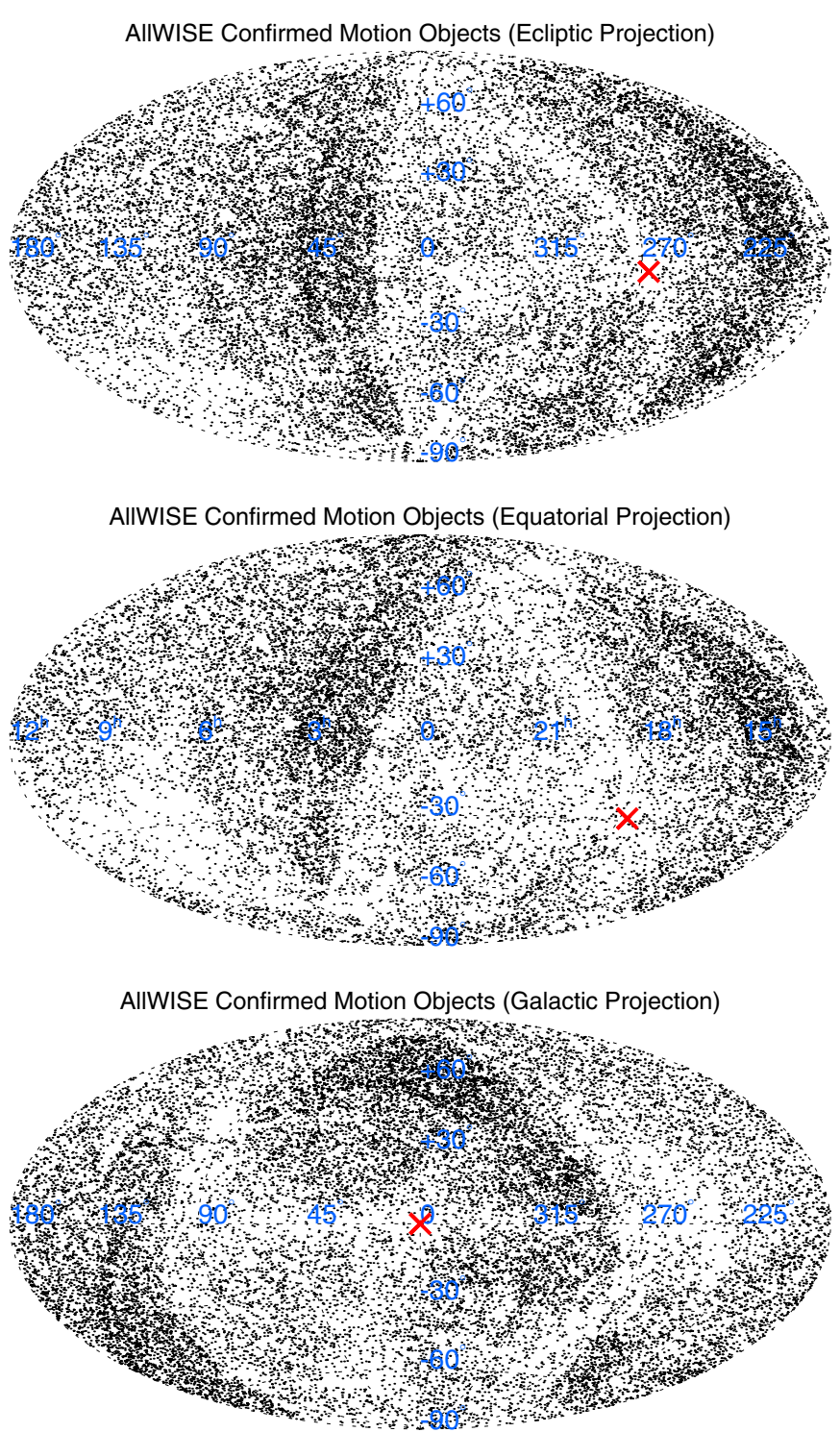

Figure 14. All-sky maps showing the locations of the 22,387 confirmed motion objects identified in AllWISE. Of these, 18,862 are rediscoveries whose motions are given in SIMBAD and the other 3525 are new discoveries from Table 3 . The projections shown are (top) ecliptic, (middle) equatorial, and (bottom) Galactic. A red "X" marks the location of the Galactic Center.

(A color version of this figure is available in the online journal.)

source has an entry in another motion catalog, even though the source itself has no publication history, and Column 12 indicates whether additional information is available in the footnotes.

The 58 AllWISE motion candidates lacking counterparts in 2MASS and other earlier surveys are listed in Table 4. Sixty percent of the objects in this table (35 out of 58) are believed to be flux transients based on their blue $W 1-W 2$ colors and appearance on the WISE All-Sky Release and AllWISE Release Atlas Images. These objects are slightly fainter versions of objects clearly seen in the $W 1$ individual frames at one, and only one, WISE epoch, but because of their faintness, the variability cannot be confirmed by eye using the individual frames. Most of the remaining $40 \%$ of the objects may or may not be real motion objects. One of these, WISEA J085510.74-071442.5, was also found by Luhman (2014), and the AllWISE motion values of $-4188 \pm 267$ and $226 \pm 283$ mas yr $^{-1}$ in R.A. and Decl., respectively, match well with his measures of $-4800 \pm 300$ and 

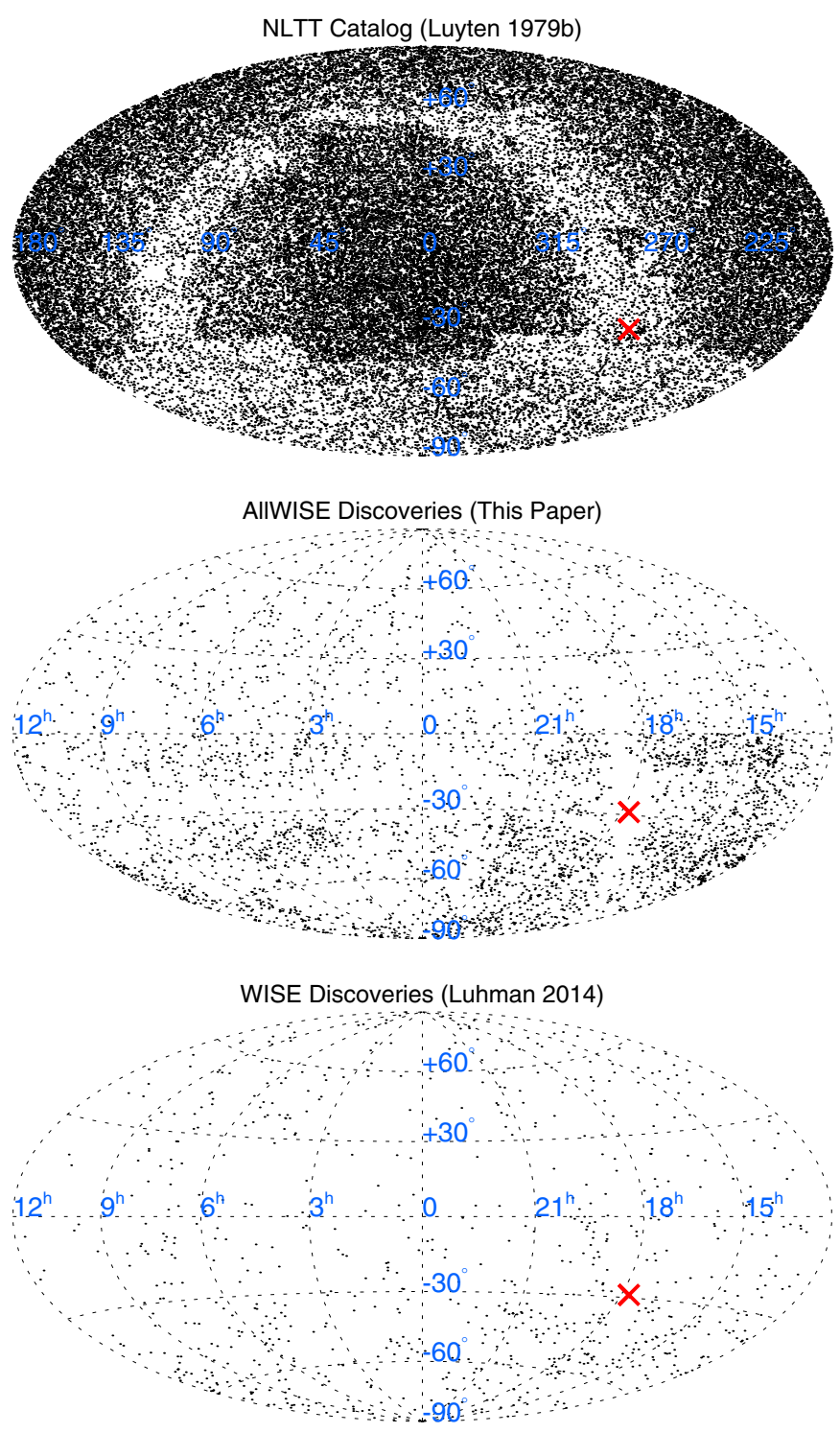

Figure 15. All-sky maps showing the locations of (top) the 58,845 motion objects from the New Luyten Two Tenths Catalog (Luyten 1979b), (middle) the 3525 motion objects from Table 3 of this paper, and (bottom) the 762 motion objects from Luhman (2014). These are equatorial coordinate projections with the vernal equinox at the center and R.A. increasing to the left. A red "X" marks the location of the Galactic Center.

(A color version of this figure is available in the online journal.)

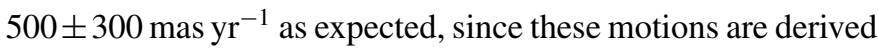
from the same data set. Visual inspection of the individual frames also strongly suggests a large motion between the two WISE epochs, but we nonetheless list this source as unconfirmed pending a third-epoch verification.

The next set of plots aim to characterize objects in Table 3. Figure 16 shows the AllWISE-measured motions plotted against the 2MASS-to-WISE motions. Careful analysis of the left and middle panels of this plot shows that, unlike the truth-test cases in Figure 6, the points do not fall along the line of one-to-one correspondence but rather would have a fitted slope much steeper than the one-to-one line. The reason for this is an inherent bias in our selection criteria. By demanding that the motion value be statistically significant compared to its measured errors means that at a given value of R.A. or Decl. motion we preferentially select those that are measured on the high side of the average rather than on the low side. This effect is also seen in the right panel of Figure 16. As expected given this bias, the AllWISE-measured motions tend to be higher than those measured independently.

Figure 17 shows the AllWISE $W 1$ profile-fit magnitude of each source plotted against the total motion computed from the 2MASS-to-WISE time baseline. As can be seen on the figure, 10 of these objects have motions $\geqslant 1000$ mas $y r^{-1}$, including the three highest movers WISEA J204027.30+695924.1 ( $\mu=2300 \pm 10$ mas yr $^{-1}$; see Section 7), WISEA J154045.67-510139.3 $\left(\mu=2006 \pm 12\right.$ mas yr $^{-1}$; see Section 8), and WISEA J070720.50+170532.7 $(\mu=1802 \pm$

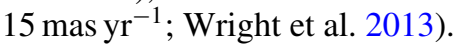

Figure 18 shows the 2MASS $J$-band magnitude plotted as a function of the $J-W 2$ color. As shown in Figure 7 of Kirkpatrick et al. (2011), L and T dwarfs generally have colors of $J-W 2>2.0 \mathrm{mag}$, so the list of AllWISE motion objects contains approximately 30 new L and T dwarfs. Figure 19 (which can be compared to Figure 9 in Kirkpatrick et al. 2011) breaks the degeneracy between the L and T dwarf populations. Figure 1 of Kirkpatrick et al. (2011) shows that the break between the $\mathrm{L}$ and $\mathrm{T}$ dwarf populations is near $W 1-W 2=0.6 \mathrm{mag}$, which means that of the $\sim 30$ new L and T dwarfs, only three are red enough to be $\mathrm{T}$ dwarfs themselves. These objects are WISEA J011154.36-505343.4, WISEA J210529.11-623559.3, and WISEA J212100.87-623921.6, all of which lack previous publication, likely because their very southern declinations have made follow-up somewhat more difficult.

None of these objects is nearly red enough to fall in the $\mathrm{Y}$ dwarf regime, which falls very roughly at $W 1-W 2>3.5$ mag. It is natural, then, to ask why the AllWISE motion survey wasn't more efficient at picking up $\mathrm{T}$ and $\mathrm{Y}$ dwarfs. There are two reasons. First, the apparent magnitudes of the nearest $\mathrm{T}$ and $\mathrm{Y}$ dwarfs are sufficiently faint that AllWISE would only have been able to measure significant motions for the very fastest movers (see Figure 5). Second, several bright, very high motion $\mathrm{T}$ and $\mathrm{Y}$ dwarfs were picked up by our criteria, but these are not included in Table 3 because they were previously known. In fact, it is hard to imagine a bright, nearby $\mathrm{T}$ or $\mathrm{Y}$ dwarf that would not have been picked up via color selection because, after all, WISE was designed to be highly sensitive to the methane absorption that defines these spectral types. With longer time baselines, WISElike data would be able to probe deeper magnitude limits and smaller motions, enabling the discovery of fainter and/or colder objects than color selection itself can provide. Although it is possible that AllWISE has identified a moving W2-only source with a $W 1-W 2$ color limit sufficiently marginal that colorbased criteria would not have selected it, our current motionbased criteria did not extract it. Modifying the selection criteria will be further discussed in Section 5 .

Finally, we present two plots that help to isolate potentially low-metallicity objects. Figure 20 shows the reduced proper motion at $J$ band plotted against the $J-W 2$ color. The reduced proper motion can be thought of as a poor man's substitute for an absolute magnitude measurement when a parallax is lacking. In the distance modulus equation, the total proper motion is used in place of the parallax (this is done because, to first order, the higher an object's proper motion, the larger its parallax is likely to be) and the "absolute magnitude" that results is called the reduced proper motion. At $J$ band, this would be written as $H_{J}=J+5 \log (\mu)+5$, where $\mu$ is expressed in arcsec $\mathrm{yr}^{-1}$ and the reduced proper motion is in magnitudes. Objects with abnormally high space velocities confound this 

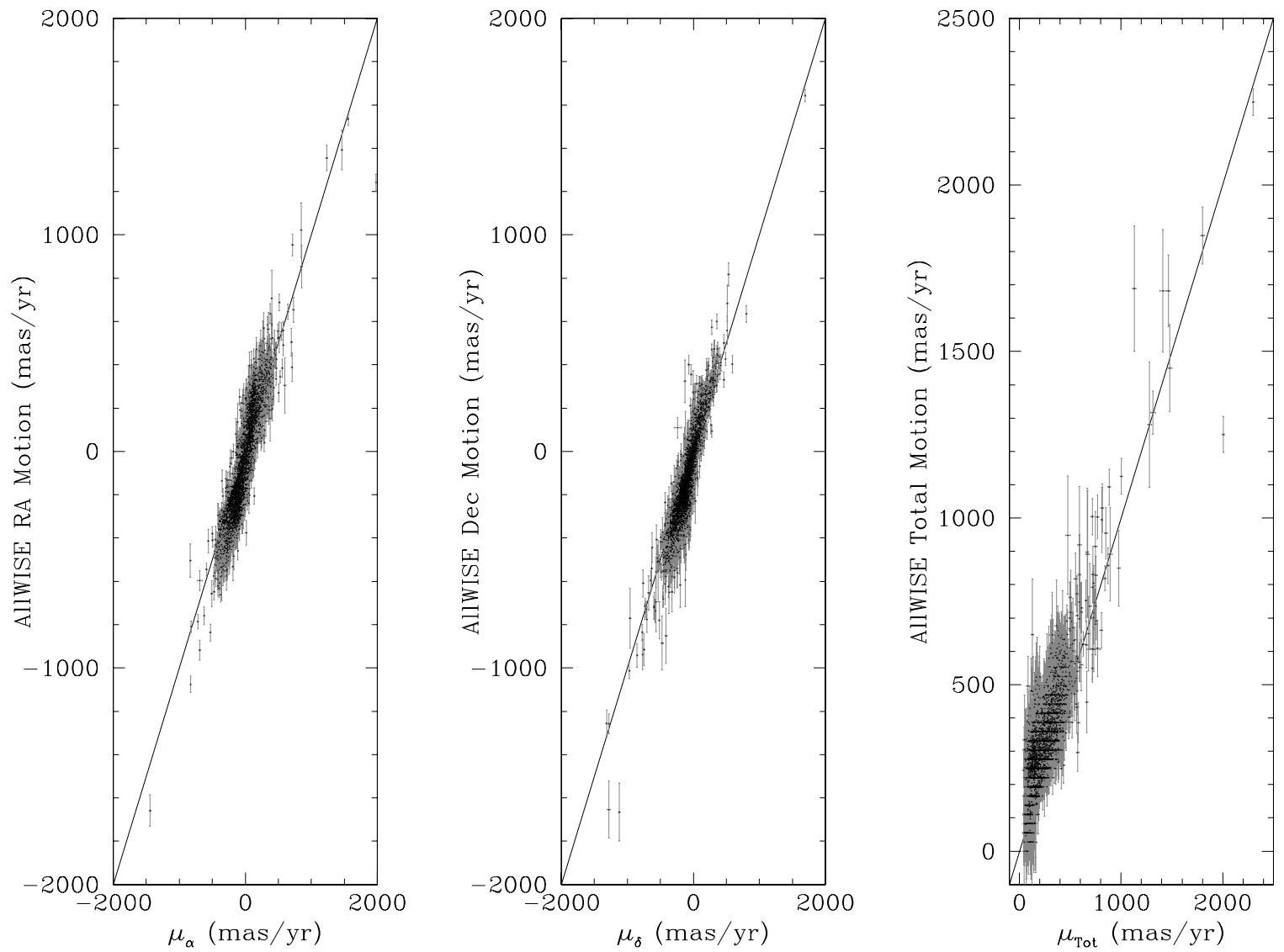

Figure 16. AllWISE-measured R.A. (left), Decl. (middle), and total (right) motions plotted against proper motions measured using the 2MASS-to-WISE time baseline for objects in Table 3. The line of one-to-one correspondence is shown in each panel.

logic, however-they have high motions not because they are close but because they are old. As a result, these objects have reduced motion values that are significantly fainter than objects of average kinematics (see Reid 1997 for a more extensive discussion). Fortunately, this enables such objects (which tend to be low-metallicity because they are very old) to be easily selected from the bulk of objects (of near solar metallicity) in the Galactic disk. Several objects with reduced proper motion at $J$ band $>19 \mathrm{mag}$ and $0.8<J-W 2<1.5 \mathrm{mag}$ can be seen in Figure 20 and are prime low-metallicity candidates.

Figure 21 shows the $J-K_{s}$ color plotted against the $J-W 2$ color. The locus at lower left near $(0.3,0.3)$ corresponds roughly to late-F or early-G spectral types (Ali 2014) and marks the earliest spectral types for which we have new motions. The bulk of objects clustered near $(1.1,0.8)$ are mid-M dwarfs, which by far dominate the solar neighborhood (Figure 11 of Kirkpatrick et al. 2012). The trail of objects into the upper right corner of the figure is the $\sim 30 \mathrm{~L}$ and $\mathrm{T}$ dwarfs. This diagram also shows a collection of objects that fall below the standard main sequence. Qualitatively, if we take the long-baseline color, $J-W 2$, to be indicative of temperature or spectral type, and the short-baseline color, $J-K_{s}$ to sample more specific atmospheric physics, we would expect objects at the same $J-W 2$ value as a standard main sequence star but with a bluer $J-K s$ value to be low-metallicity because of the increased contribution of collision-induced absorption by $\mathrm{H}_{2}$ at $K$ band. Hence, this diagram provides another handy way-using colors instead of motions, as in Figure 20-to select low-metallicity candidates.

\section{COMPARISON TO THE LUHMAN (2014) MOTION LIST}

While this paper was being finalized, Luhman (2014) published an independently selected set of motion objects uncovered in the same WISE data set. Rather than using AllWISE results, his methodology started with the publicly available single exposure catalogs from each phase of the mission. The ecliptic polar regions were ignored (to save on processing time) and the rest of the WISE data divided into discrete epochs encompassing the twelve or more revisits of the same patch of sky with an overall time difference generally less than one day. The mean coordinates of the group at a single epoch were computed, and these were compared to other groups at other epochs as long as two groups were within 1".5 of one another. Motions were thus computed for these groups paired across epochs, and groups for which no pairings were found were examined separately in case they corresponded to very high motion objects. The Luhman (2014) list contains 762 objects, and its sky distribution (lower panel of Figure 15) is similar to ours, although the source density is considerably lower. Of those 762 objects, 321 are also included in our Table 3, one (the unconfirmed object WISEA J085510.74-071442.5) is included in Table 4, and another (WISEA J104915.52-531906.1) was eliminated from consideration because it was published earlier by Luhman (2013).

The remaining 439 objects enable us to determine which of our selection criteria were set too tightly, so that future researchers can perform their own, refined searches. Some sources fail more than one of the criteria discussed in Section 4.1. 


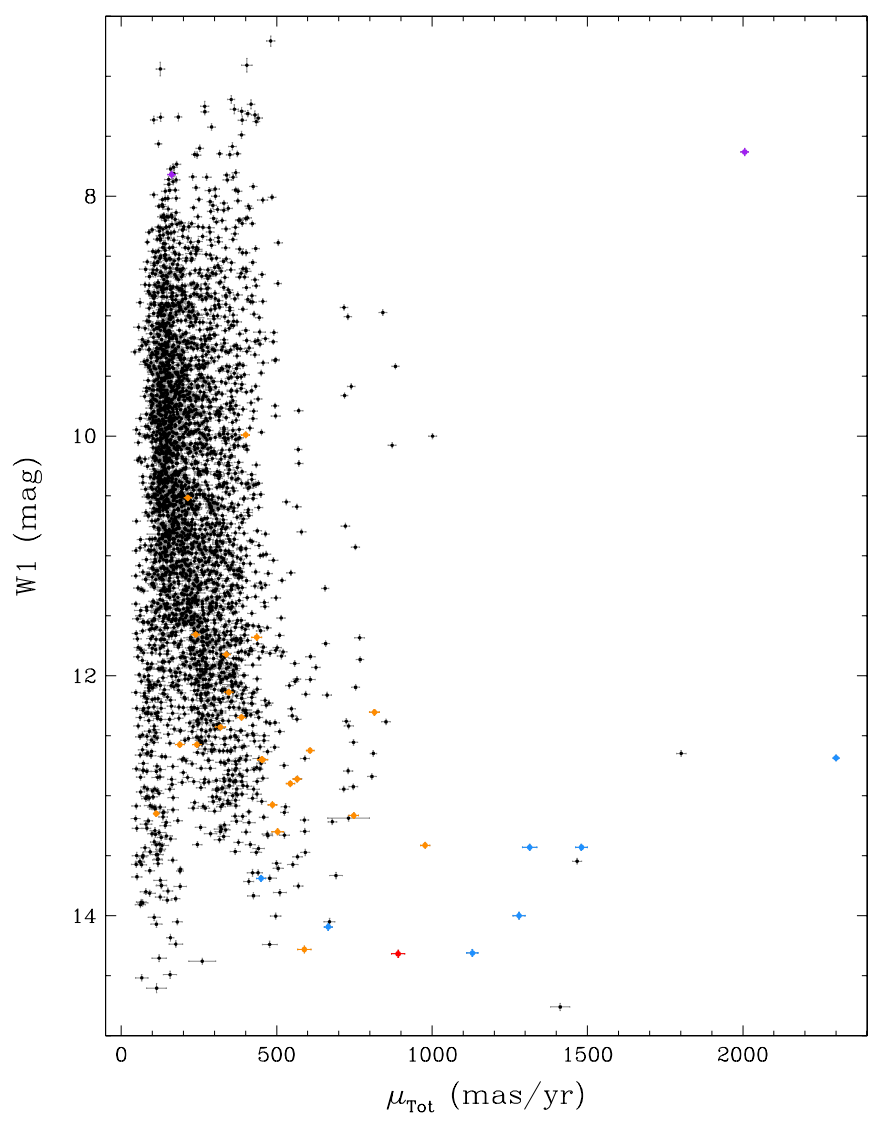

Figure 17. AllWISE $W 1$ profile-fit photometry plotted as a function of the total 2MASS-to-WISE proper motion for objects in Table 3. Objects with spectroscopic follow-up in Table 5 are color coded as follows: Light blue-earlyL subdwarfs; red-late-L subdwarfs; purple-bright $M$ dwarfs discussed in Section 8; dark orange-all others.

(A color version of this figure is available in the online journal.)

Specifically, a total of $80 \%(351 / 439)$ of the objects were missed because the $r c h i 2 / r c h i 2_{-} p m$ ratio failed to exceed a threshold of 1.03 . That is, the reduced $\chi^{2}$ from the motion fit was not markedly better than that from the stationary fit. In most cases, the reduced $\chi^{2}$ was improved, but this improvement was less than $3 \%$.

The next most problematic criteria were the restrictions that $w$ ?rchi 2 be less than 2.0 to ensure a point-like source. These failed in $39 \%(172 / 439)$ of the cases. In a similar vein, $13 \%$ $(56 / 439)$ of the sources failed the $n b=1$ criterion to assure that the source was not blended. In most of these cases, a check of the WISE images confirms that the motion source from Luhman (2014) is blended with another source and/or appears slightly elongated, but AllWISE processing was nonetheless able to handle these cases. (It should be expected, however, that there are plenty of other cases of blending and elongation that are not so elegantly handled.) Finally, 15\% (64/439) of the sources failed the $Q$ criterion, $2.5 \%$ (11/439) failed the $c c_{-}$flags criterion, $1.4 \%(6 / 439)$ failed the pmcode criterion, $1.4 \%$ (6/439) were missed during by-eye Quality Assurance reviewing (one of these was caused by a poor stretch on the images used to scrutinize candidates), $0.7 \%(3 / 439)$ were lost due to transcription errors, and $0.2 \%(1 / 439)$ is a previously published source that was removed from our list because it was not a new discovery.

Figure 22 compares the Luhman (2014) motion objects (760 total after removing WISEA J085510.74-071442.5 and WISEA J104915.52-531906.1) to our list of 3525 objects with

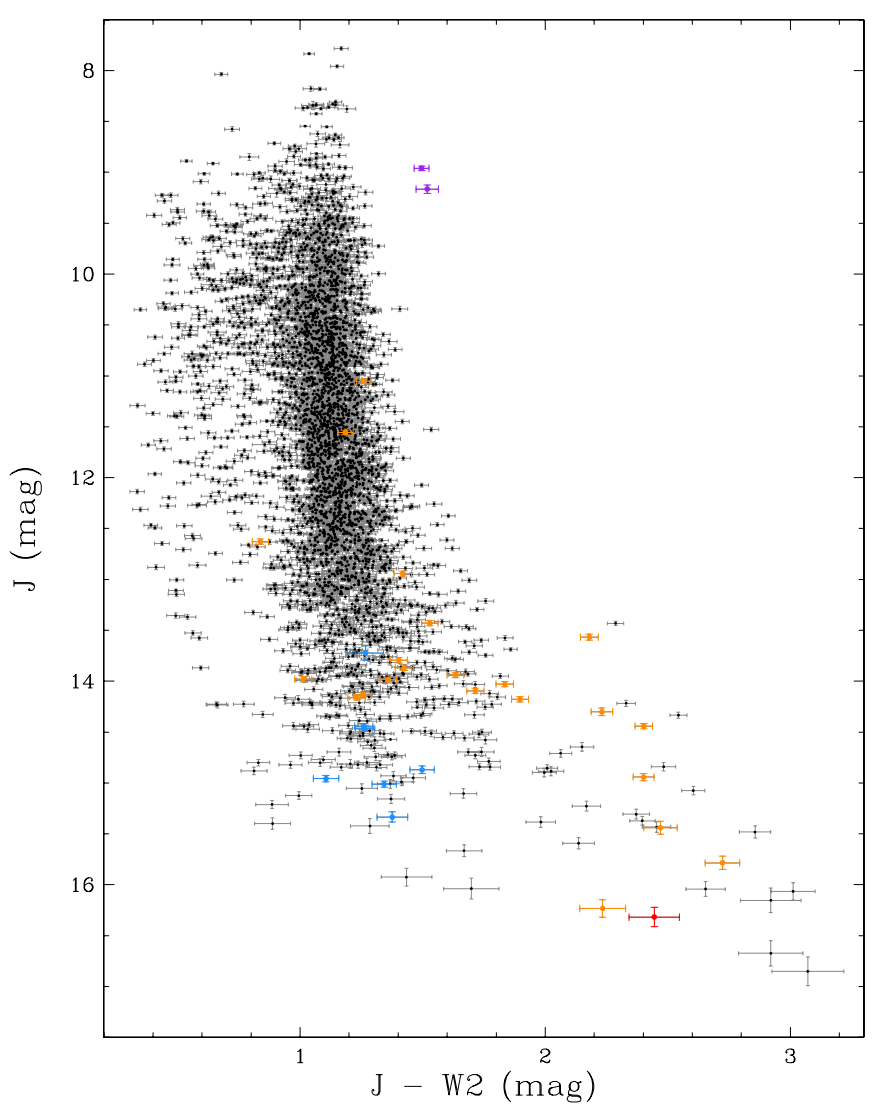

Figure 18. 2MASS $J$-band magnitude plotted against the $J-W 2$ color for the motion objects in Table 3. Color coding is the same as in Figure 17.

(A color version of this figure is available in the online journal.)

confirmed motions (Table 3). The plot shows histograms in $W 1$ magnitude and in total proper motion computed from the 2MASS-to-WISE time baseline. Our survey tends to sample brighter objects than the Luhman (2014) catalog and, as a result, also probes to considerably smaller motion. Both surveys are able to select high motion objects, although there are somewhat

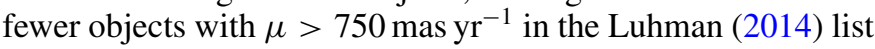
than in ours.

Our criteria missed objects found by Luhman (2014), but Luhman (2014) also missed objects found by us. Our secondhighest motion object, WISEA J154045.67-510139.3, is one notable example of the latter case. Clearly, the AllWISE data products hold many other potential discoveries. Moreover, with the WISE satellite having been reactivated in 2013 December for an additional three years of data taking, extending this all-sky motion survey to even smaller motion limits via the extended time baseline will also be possible. Such a survey would allow researchers to expand the volume and spectral type range over which WISE motion discoveries can be made.

\section{SPECTROSCOPIC FOLLOW-UP OF SELECTED DISCOVERIES}

We have selected objects for spectroscopic observation as follows. Figure 17 was used primarily to select objects of highest motion, since these tend to be either close or low-metallicity. Figure 18 was used to look for objects that were bright in $J$-band magnitude relative to their peers in $J-W 2$ color, since such objects are generally the closest objects of their type. Figure 19 was used to select late-type objects, although all of the T dwarf 


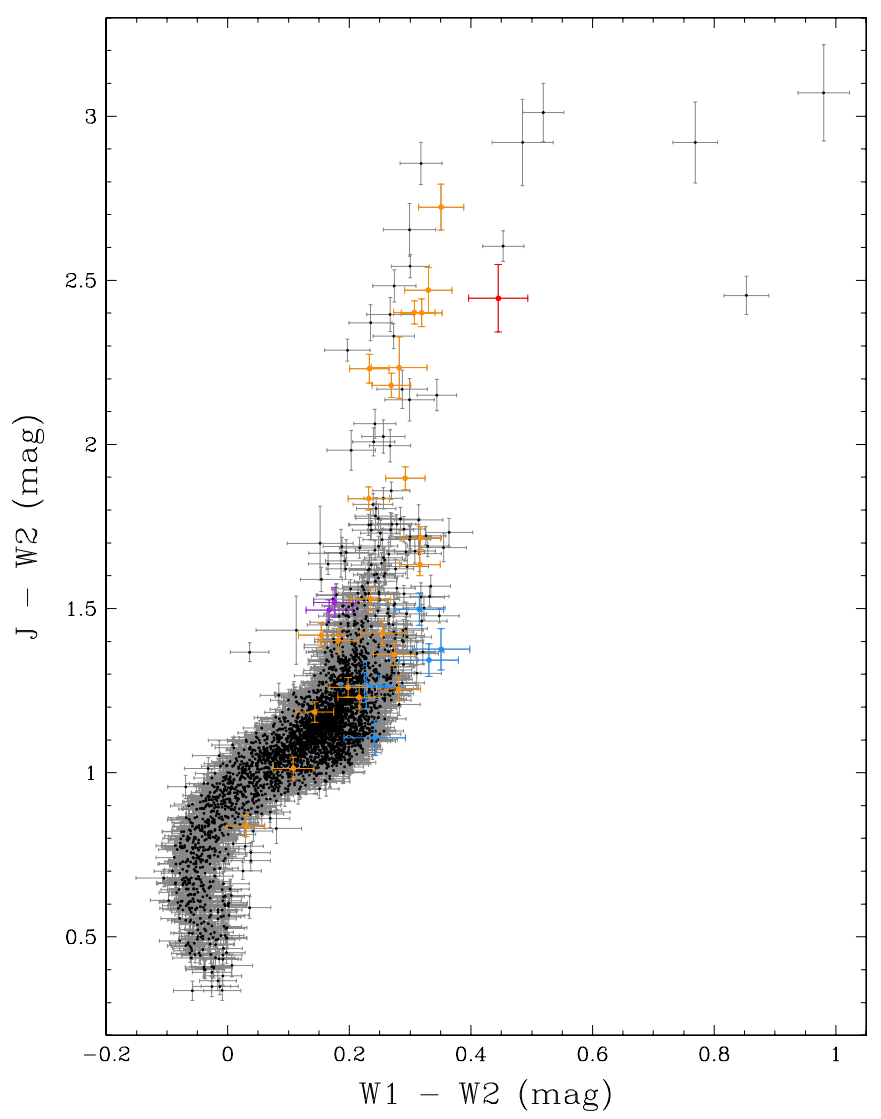

Figure 19. $J-W 2$ color vs. $W 1-W 2$ color for the motion objects in Table 3 . Color coding is the same as in Figure 17.

(A color version of this figure is available in the online journal.)

candidates were at unfavorable sky positions for the followup obtained for this paper. Figures 20 and 21 were used to select low-metallicity objects since, as explained earlier, such objects tend to fall in the lower parts of these diagrams, thereby distinguishing themselves from the bulk of objects, which have solar metallicities. Objects for which we have obtained spectroscopic follow-up are color coded in Figures 17-21, as described in the legend to Figure 17. These follow-up observations are detailed below and summarized in Table 5.

\subsection{Optical Spectroscopy}

One source was observed (by S.F.A.) on the night of 2013 July 07 UT with the Double Spectrograph on the Hale 5m telescope on Palomar Mountain, California. In the red-side spectrograph, a 316 lines $\mathrm{mm}^{-1}$ grating blazed at $7500 \AA$ was used to produce a spectrum covering the range $3800-10500 \AA$. Standard reduction procedures for CCD data were employed. This night had cirrus clouds.

Six sources were observed (by D.S., M.B., and G.B.L.) on the nights of 2013 October 4-5 UT with the Low Resolution Imaging Spectrometer (LRIS; Oke et al. 1995) at the $10 \mathrm{~m} \mathrm{~W}$. M. Keck Observatory on Mauna Kea, Hawai'i. The blue side was used with a 600 lines $\mathrm{mm}^{-1}$ grating blazed at $4000 \AA$, and the red side was used with a 400 lines $\mathrm{mm}^{-1}$ grating blazed at $8500 \AA$. Objects had very little flux in the blue side so the blueside spectra are not considered further. The red side produced a spectrum covering the range from 5500 to $10000 \AA$. Standard reduction procedures for $\mathrm{CCD}$ data were employed. The first night had clouds and the second night was clear.

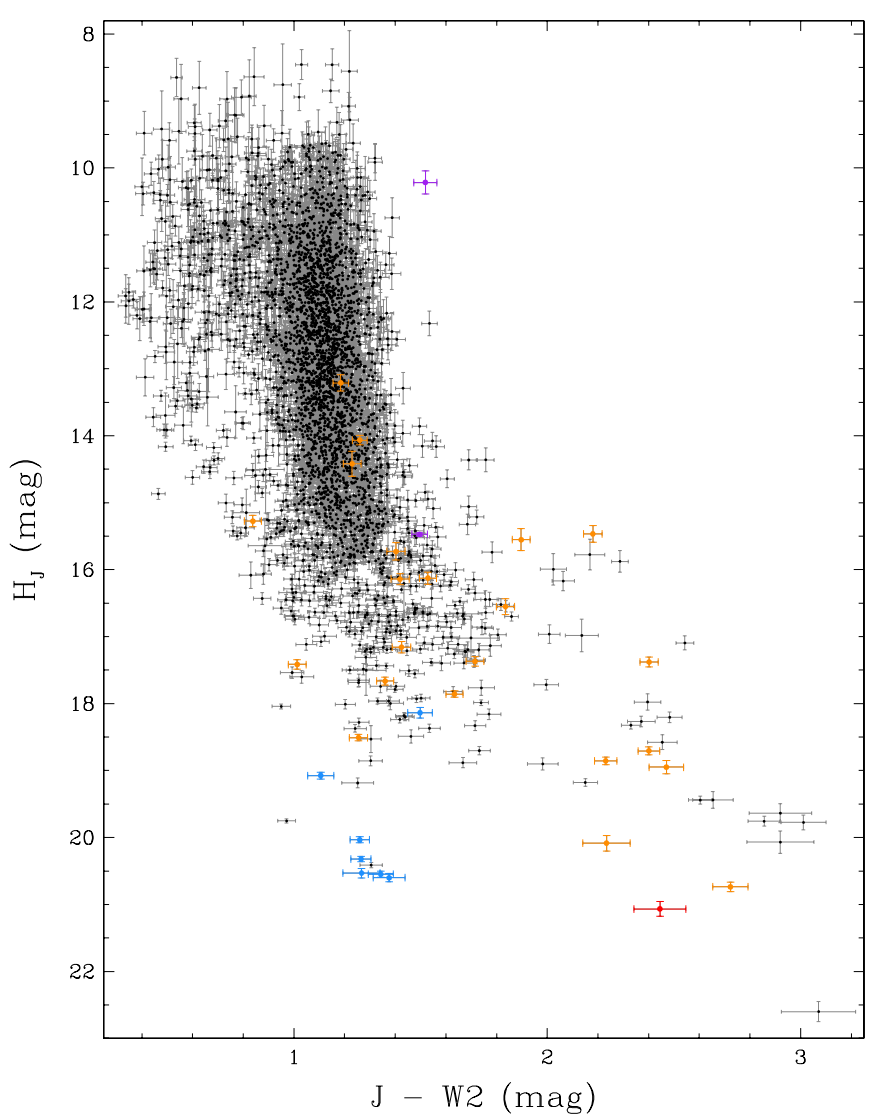

Figure 20. Reduced proper motion at $J$ band plotted against the $J-W 2$ color for the motion objects in Table 3. Color coding is the same as in Figure 17. (A color version of this figure is available in the online journal.)

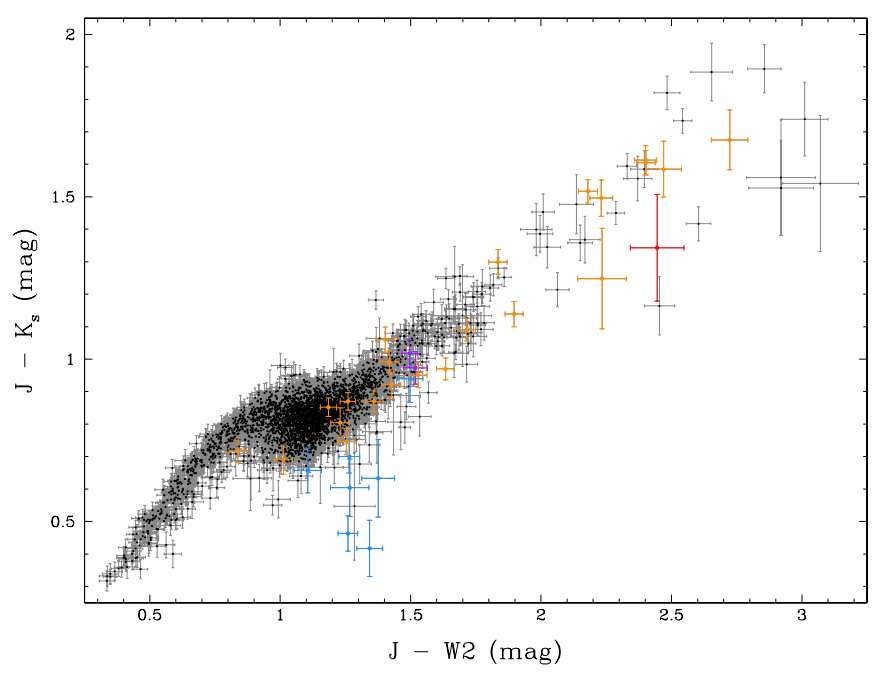

Figure 21. $J-K_{s}$ color plotted against the $J-W 2$ color for the motion objects in Table 3. Color coding is the same as in Figure 17.

(A color version of this figure is available in the online journal.)

Three sources were observed (by D.S.) on the clear night of 2013 December 11 UT with the Deep Imaging Multi-object Spectrograph (DEIMOS; Faber et al. 2003) at the $10 \mathrm{~m} \mathrm{W.} \mathrm{M.}$ Keck Observatory on Mauna Kea, Hawai'i. The instrument was used in single-object mode utilizing the 1200 line $\mathrm{mm}^{-1}$ grating blazed at $7500 \AA$ to provide continuous wavelength coverage from 6650 to $9300 \AA$. Standard reduction procedures for CCD data were employed. 

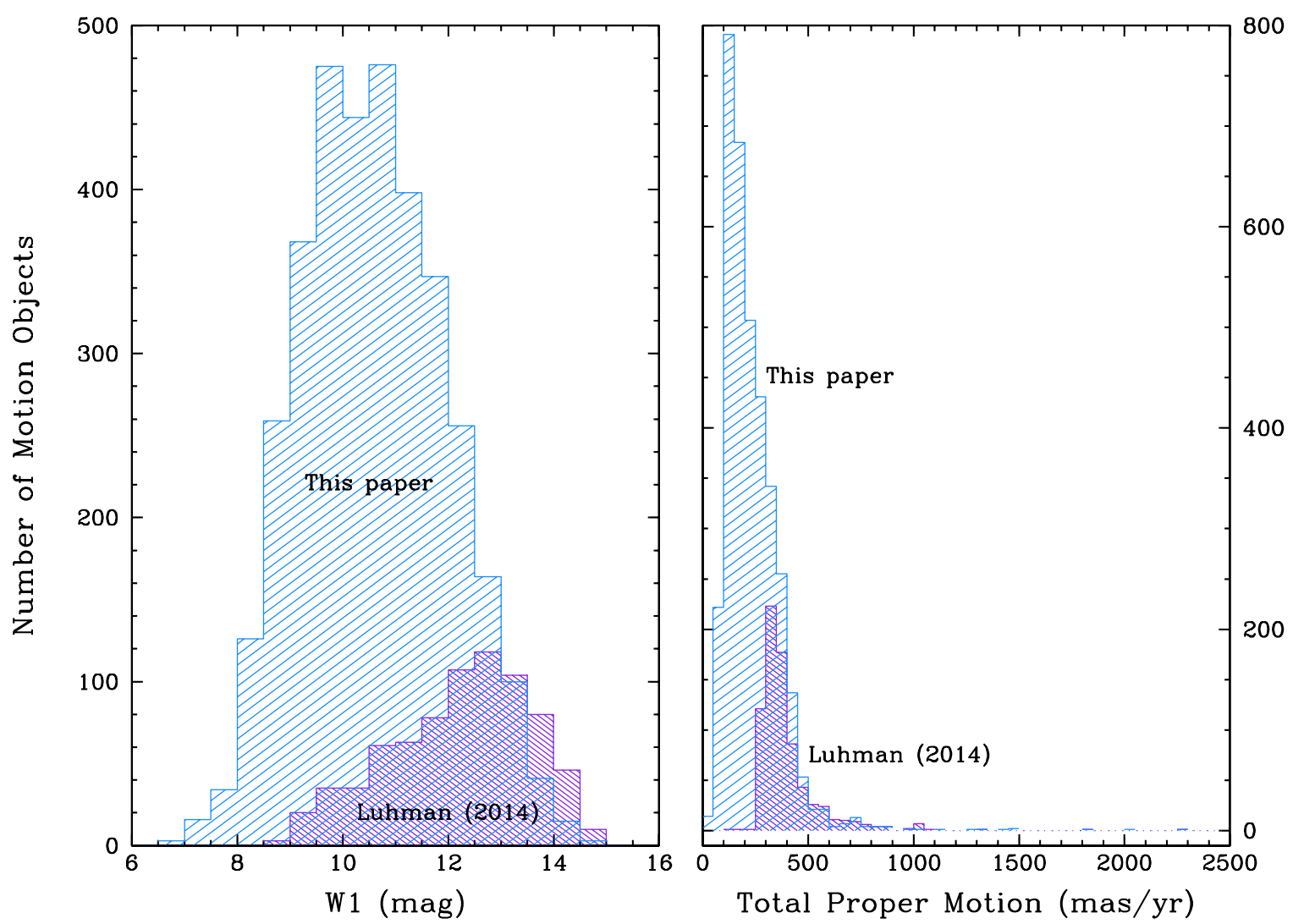

Figure 22. Histograms of the $W 1$ mag (left panel) and total 2MASS-to-WISE proper motion (right panel) for our survey (light blue; 3525 objects from Table 3) compared to that of Luhman (2014; violet; 760 objects total).

(A color version of this figure is available in the online journal.)

Two objects were observed (by J.A.R.) on the clear nights of 2014 January 27 and 28 UT with the Inamori-Magellan Areal Camera and Spectrograph (IMACS; Dressler et al. 2011) on the $6.5 \mathrm{~m}$ Walter Baade Telescope at Las Campanas Observatory, Chile. The f $/ 4$ camera was used with a Bessel $V$-band or a CTIO $I$-band filter for imaging observations and with a 600 lines $\mathrm{mm}^{-1}$ grating for the spectroscopic observations. The grating angle was set for a wavelength coverage of 7100-10400 $\AA$. Standard reduction procedures for $\mathrm{CCD}$ data were again employed.

\subsection{Near-infrared Spectroscopy}

Several sources were observed with SpeX (Rayner et al. 2003) on the NASA 3m Infrared Telescope Facility (IRTF) on Mauna Kea, Hawai'i. The UT dates of observation were 2013 August 14 (by A.J.B.; cirrus and patchy clouds), 2013 October 24 (by A.S.; clear skies), 2013 November 21-22 and 24 (by J.K.F. and N.S.; thick cirrus on the first night but then clear), and 2013 December 14-15 (by A.S.; clear). For all but the October night, SpeX was used in prism mode with a $0^{\prime \prime} .5$ or $0^{\prime \prime} .8$ wide slit to achieve a resolving power of $R \equiv \lambda / \Delta \lambda \approx 100-150$ over the range $0.8-2.5 \mu \mathrm{m}$. For the October run, SpeX was used in crossdispersed mode to produce spectra over the range 0.9-2.4 $\mu \mathrm{m}$ with a resolving power of $R \equiv \lambda / \Delta \lambda \approx 1200$. All data were reduced using Spextool (Cushing et al. 2004); A0 stars were used for the telluric correction and flux calibration steps following the technique described in Vacca et al. (2003).

Several sources were also observed on the UT nights of 2013 November 20 and 2013 December 14 (by G.N.M. and S.E.L.) with the Near-Infrared Spectrometer (NIRSPEC; McLean et al. 1998, 2000) at the $10 \mathrm{~m}$ W. M. Keck Observatory on Mauna Kea, Hawai'i. There was cirrus on the November night and heavier clouds on the December one. In low-resolution mode, use of the $42^{\prime \prime} \times 0$ '.38 slit results in a resolving power of $\mathrm{R} \equiv \lambda / \Delta \lambda \approx 2500$. Our brown dwarf candidates were observed in the N3 configuration (see McLean et al. 2003) that covers part of the $J$-band window from 1.15 to $1.35 \mu \mathrm{m}$. Data were reduced using the REDSPEC package, as described in McLean et al. (2003).

\subsection{Spectral Classification}

We have typed the optical spectra as follows. Each spectrum was normalized at $7500 \AA$ or $8250 \AA$ and overplotted on a suite of like-normalized LRIS spectra of primary $\mathrm{M}$ and L optical spectral standards from Kirkpatrick et al. (1991) and Kirkpatrick et al. (1999). These plots were examined by eye to determine the best match and to look for any peculiarities with respect to the standard sequence. Objects falling midway between integral classes (such as M9 and L0) were assigned the half class in between (in this case, M9.5). Objects showing notable peculiarities were given a suffix of "pec" unless the peculiarities were determined to be caused by low-metallicity, in which case these were reclassified against published optical spectra typed as late-M and early-L subdwarfs and given a prefix of "sd."

We have typed the near-infrared spectra as follows. Each target spectrum was normalized to one at $1.28 \mu \mathrm{m}$ and compared to the near-infrared spectral standards from Kirkpatrick et al. (2010) normalized the same way. Using the methodology outlined in that paper, the core near-infrared type was determined only from the $0.9-1.4 \mu \mathrm{m}$ portion, and then the corresponding goodness of fit to the same spectral standard from 1.4-2.5 $\mu \mathrm{m}$ was judged. In most cases the same spectral standard also 
Table 5

Spectroscopic Follow-up of Motion Candidates

\begin{tabular}{|c|c|c|c|c|c|c|}
\hline Object Name & $\begin{array}{c}\text { Opt } \\
\text { Sp. Type } \\
\text { (2) }\end{array}$ & $\begin{array}{c}\text { NIR } \\
\text { Sp. Type } \\
\text { (3) }\end{array}$ & $\begin{array}{c}\text { Telescope/ } \\
\text { Instrument } \\
\text { (4) }\end{array}$ & $\begin{array}{l}\text { Obs. Date } \\
\text { (UT) } \\
(5)\end{array}$ & $\begin{array}{c}\text { Exp. Time }{ }^{\mathrm{a}} \\
\text { (s) } \\
(6)\end{array}$ & $\begin{array}{l}\text { Corrector } \\
\text { Star } \\
(7)\end{array}$ \\
\hline WISEA J001450.17-083823.4 & sdL0 & 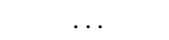 & Keck/DEIMOS & 2013 Dec 11 & 600 & \\
\hline WISEA J004326.26+222124.0 & $\ldots$ & sdL1 & IRTF/SpeX & 2013 Oct 24 & 960 & HD 7215 \\
\hline WISEA J004713.81-371033.7 & L4: & $\ldots$ & Keck/DEIMOS & 2013 Dec 11 & 600 & $\ldots$ \\
\hline WISEA J005757.64+201304.0 & sdL7 & & Keck/LRIS & 2013 Oct 5 & $1167 / 1130$ & \\
\hline$\ldots$ & .. & sdL7 & IRTF/SpeX & 2013 Nov 24 & 1800 & HD 16811 \\
\hline WISEA J020201.25-313645.2 & sdL0 & $\ldots$ & Keck/LRIS & 2013 Oct 5 & $600 / 600$ & $\ldots$ \\
\hline WISEA J023421.83+601227.3 & M9.5e & $\ldots$ & Palomar/DSpec & $2013 \mathrm{Jul} 7$ & 2900 & $\ldots$ \\
\hline WISEA J030601.66-033059.0 & sdL0 & $\ldots$ & Keck/DEIMOS & 2013 Dec 11 & 300 & $\ldots$ \\
\hline a & $\ldots$ & sdL0 & IRTF/SpeX & 2013 Nov 22 & 720 & HD 13936 \\
\hline WISEA J040418.01+412735.5 & $\ldots$ & L3 pec (red) & IRTF/SpeX & 2013 Oct 24 & 720 & HD 21038 \\
\hline WISEA J043535.82+211508.9 & sdL0 & $\ldots$ & Keck/LRIS & 2013 Oct 4 & $300 / 300$ & $\ldots$ \\
\hline$\ldots$ & $\ldots$ & sdL0 & IRTF/SpeX & 2013 Oct 24 & 960 & HD 27761 \\
\hline 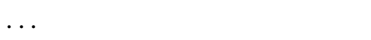 & $\ldots$ & sdL0 & Keck/NIRSPEC & 2013 Dec 14 & 2400 & HD 35036 \\
\hline WISEA J045921.21+154059.2 & $\ldots$ & sdL0 & IRTF/SpeX & 2013 Nov 22 & 360 & HD 35036 \\
\hline WISEA J053257.29+041842.5 & $\ldots$ & L3 & Keck/NIRSPEC & 2013 Nov 20 & 720 & HD 39953 \\
\hline WISEA J054318.95+642250.2 & $\ldots$ & L2 & Keck/NIRSPEC & 2013 Dec 14 & 1440 & HD 33654 \\
\hline WISEA J055007.94+161051.9 & $\ldots$ & $\mathrm{L} 2$ & IRTF/SpeX & 2013 Nov 24 & 720 & HD 43583 \\
\hline WISEA J060742.13+455037.0 & $\ldots$ & $\mathrm{L} 2.5$ & Keck/NIRSPEC & 2013 Dec 14 & 1800 & HD 45105 \\
\hline$\ldots$ & $\ldots$ & $\mathrm{L} 2.5$ & IRTF/SpeX & 2013 Dec 15 & 960 & HD 45105 \\
\hline WISEA J063957.71-034607.2 & sdM0.5 & $\ldots$ & Keck/LRIS & 2013 Oct 4 & $300 / 300$ & \\
\hline WISEA J065958.55+171710.9 & $\ldots$ & L2 & Keck/NIRSPEC & 2013 Nov 20 & 960 & HD 39953 \\
\hline WISEA J071552.38-114532.9 & $\ldots$ & L5 pec? & Keck/NIRSPEC & 2013 Nov 20 & 960 & HD 43607 \\
\hline$\ldots$ & $\ldots$ & L4 pec (blue) & IRTF/SpeX & 2013 Dec 14 & 960 & HD 56525 \\
\hline WISEA J072003.20-084651.3 & $\ldots$ & M9 & IRTF/SpeX & 2013 Dec 14 & 960 & HD 56525 \\
\hline WISEA J074211.69-121151.6 & $\ldots$ & $<\mathrm{M} 0$ (wd?) & IRTF/SpeX & 2013 Nov 21 & 1800 & HD 67725 \\
\hline WISEA J082640.45-164031.8 & $\ldots$ & L9 & Keck/NIRSPEC & 2013 Dec 14 & 2400 & HD 72282 \\
\hline WISEA J085224.36+513925.5 & $\cdots$ & M7 & IRTF/SpeX & 2013 Dec 15 & 960 & HD 45105 \\
\hline WISEA J091657.18-112104.7 & $\ldots$ & M9 & IRTF/SpeX & 2013 Dec 15 & 960 & HD 45105 \\
\hline WISEA J102304.04+155616.4 & $\ldots$ & M8 pec & IRTF/SpeX & 2013 Dec 14 & 960 & HD 89239 \\
\hline WISEA J154045.67-510139.3 & M6 & $\ldots$ & Magellan/IMACS & 2014 Jan 27 & 100 & $\ldots$ \\
\hline WISEA J162702.28-694411.8 & M4+M4 & $\ldots$ & Magellan/IMACS & 2014 Jan 28 & 300 & $\ldots$ \\
\hline WISEA J163605.71-044013.8 & $\ldots$ & M4.5 & IRTF/SpeX & 2013 Aug 14 & 120 & HD 159008 \\
\hline WISEA J182121.91-070008.6 & M7 pec & $\ldots$ & Keck/LRIS & 2013 Oct 4 & $400 / 350$ & $\ldots$ \\
\hline WISEA J204027.30+695924.1 & sdL0 & $\ldots$ & Keck/LRIS & 2013 Oct 5 & $300 / 300$ & $\ldots$ \\
\hline WISEA J204218.13-082137.8 & $\ldots$ & M7 & IRTF/SpeX & 2013 Oct 24 & 960 & HD 193689 \\
\hline WISEA J211543.59-322540.4 & $\ldots$ & M6: & IRTF/SpeX & 2013 Aug 14 & 960 & HD 199090 \\
\hline WISEA J222013.75-361709.5 & $\ldots$ & M8 pec & IRTF/SpeX & 2013 Oct 24 & 960 & HD 194272 \\
\hline WISEA J224128.33+043459.3 & $\ldots$ & M7.5 & IRTF/SpeX & 2013 Oct 24 & 960 & HD 210501 \\
\hline WISEA J232036.88+315739.5 & $\ldots$ & M4.5 & IRTF/SpeX & 2013 Oct 24 & 960 & HD 210290 \\
\hline WISEA J235459.79-185222.4 & $\ldots$ & $\mathrm{L} 2$ & Keck/NIRSPEC & 2013 Dec 14 & 1800 & HD 219833 \\
\hline
\end{tabular}

Note. ${ }^{a}$ For Keck/LRIS, the two exposure times refer to the blue-side spectrograph (left) and the red-side spectrograph (right).

provided the best fit in this longer-wavelength region. In other cases, the target spectrum was notably much bluer or redder so the fit across the $H$ and $K$ windows was very poor despite the excellent fit in the $J$ window. These peculiar objects were given suffixes of "pec (blue)" or "pec (red)" to denote the slope of the spectrum relative to the standard (see Figure 23). Some of the peculiar spectra were deemed to be low-metallicity (prefix of "sd") and were typed against near-infrared spectra of similar subdwarfs from the published literature.

Optical and/or near-infrared spectral types are listed in Table 5. Subdwarfs are discussed in Section 7, and two bright M dwarf systems are discussed in Section 8.

\section{A TROVE OF L SUBDWARF DISCOVERIES}

A number of discoveries have the photometric characteristics of subdwarfs (i.e., low-metallicity objects) and were confirmed as such by our follow-up spectroscopy. Two of these are earlyM subdwarfs-the sdM0.5 WISEA J063957.71-034607.2 and the usdM3 discovery by Wright et al. (2013) WISEA J070720.50+170532.7. Six others fall near late-M/early-L and another, WISEA J005757.64+201304.0, falls at late-L.

One of the subdwarfs near the $\mathrm{M} / \mathrm{L}$ transition is our highest motion discovery, WISEA J204027.30+695924.1. As Figure 24 shows, the optical spectrum of this object is intermediate in type between an sdM8 and an sdL1. One of the most distinctive features in the optical spectra of subdwarfs at the M/L boundary is the emission-like feature near $7050 \AA$ that is, in fact, a narrow wavelength region relatively free of opacity between the strong $\mathrm{CaH}$ band to the blue and the strong $\mathrm{TiO}$ band to the red. Optical spectra of four other AllWISE discoveries (Figure 25) show this same telltale signature and have overall spectral morphologies very similar to WISEA J204027.30+695924.1.

Note that this spectral morphology is distinctly different from that of a normal late-M dwarf (top spectrum in Figure 26), due mainly to the relatively stronger hydride bands in the subdwarfs. Only a few subdwarfs are known in this spectral range, so their 


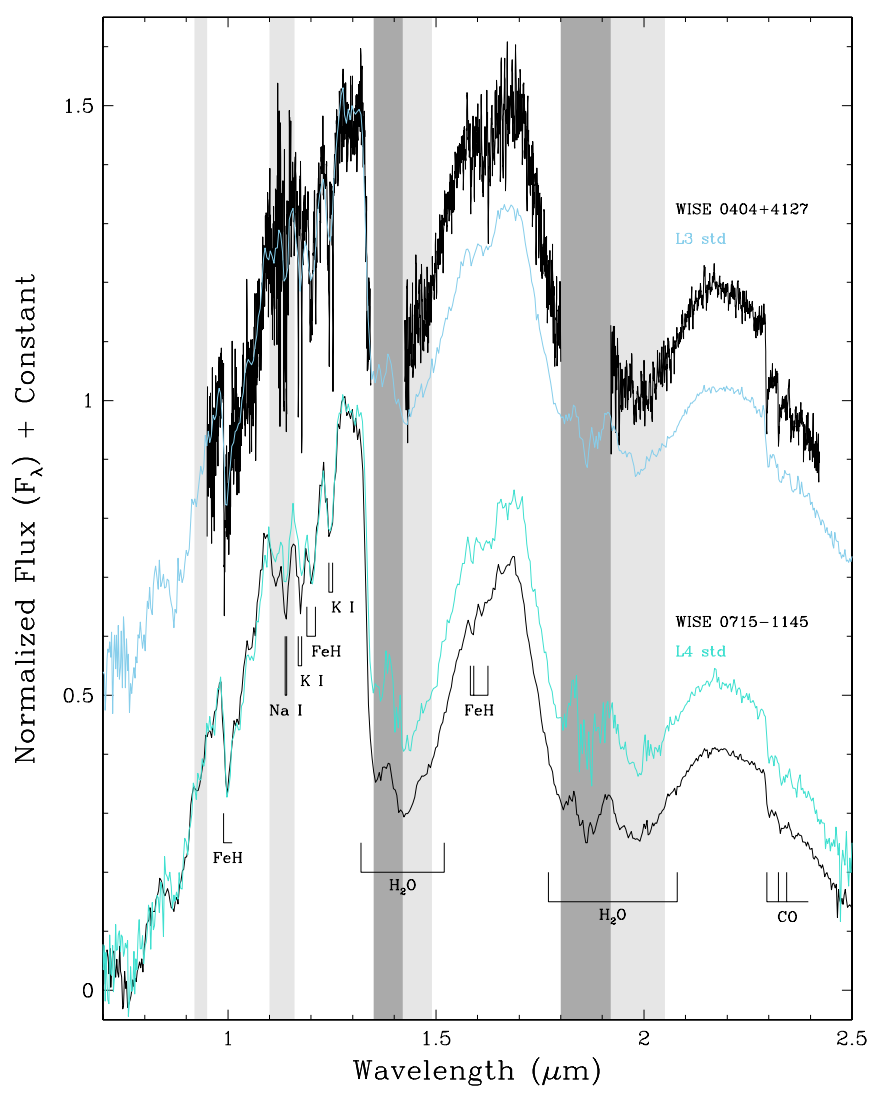

Figure 23. Near-infrared spectra of WISEA J040418.01+412735.5 (discovered earlier by Castro et al. 2013 and hence removed from our discovery list in Table 3) and WISEA J071552.38-114532.9 overplotted with the near-infrared spectral standard (Kirkpatrick et al. 2010) providing the best match throughout $J$ band. Note that the $H$ - and $K$-band spectra of WISEA J040418.01+412735.5 are much redder than the standard, and that of WISEA J071552.38-114532.9 is much bluer than the standard. Prominent spectral features are marked. Per Rayner et al. (2009), regions of telluric absorption are marked by the dark gray (atmospheric transmission $<20 \%$ ) and light gray $(20 \%<$ atmospheric transmission $<80 \%$ ) zones in wavelength. Spectra have been normalized at $1.28 \mu \mathrm{m}$ and a constant offset added to the flux to separate the spectra vertically except where overplotting was intended.

(A color version of this figure is available in the online journal.)

classification is still in its infancy. In fact, no spectroscopic standards yet exist at these types, but we can compare to other published discoveries and use their tentative types as guides. A sampling of these very late-M and early-L subdwarfs from the literature is shown by the bottom four spectra in Figure 26. There is a plateau in these spectra between 7300 and $7500 \AA$ that is bounded on the blue side by $\mathrm{TiO}$ absorption and on the red side by $\mathrm{TiO}$ and a strong $\mathrm{K}$ I doublet. The slope at the top of this plateau slowly changes from slightly red to flat through the sdM9-to-sdL0.5 sequence. That is, the flux (in units of $f_{\lambda}$ ) at the left edge of the plateau near $7300 \AA$ is lower or equal to the flux at the right edge of the plateau near $7500 \AA$. A check of the sdM8 spectrum in Figure 24 shows that the plateau has a redward slope, whereas the sdL1 spectrum in the same figure has a plateau with a blueward slope. Because all of our spectra in Figure 25 have plateaus with flat or slightly blueward slopes, we choose to classify all of these as sdL spectra. None, however have a spectral morphology as extreme as the sdL1 in Figure 24 and look more similar to the sdM9-to-sdL0.5 sequence in Figure 26. Thus, we tentatively classify all five of these spectra as sdL0.

Near-infrared spectra of four of the AllWISE motion discoveries are shown in Figure 27 and are compared with near-

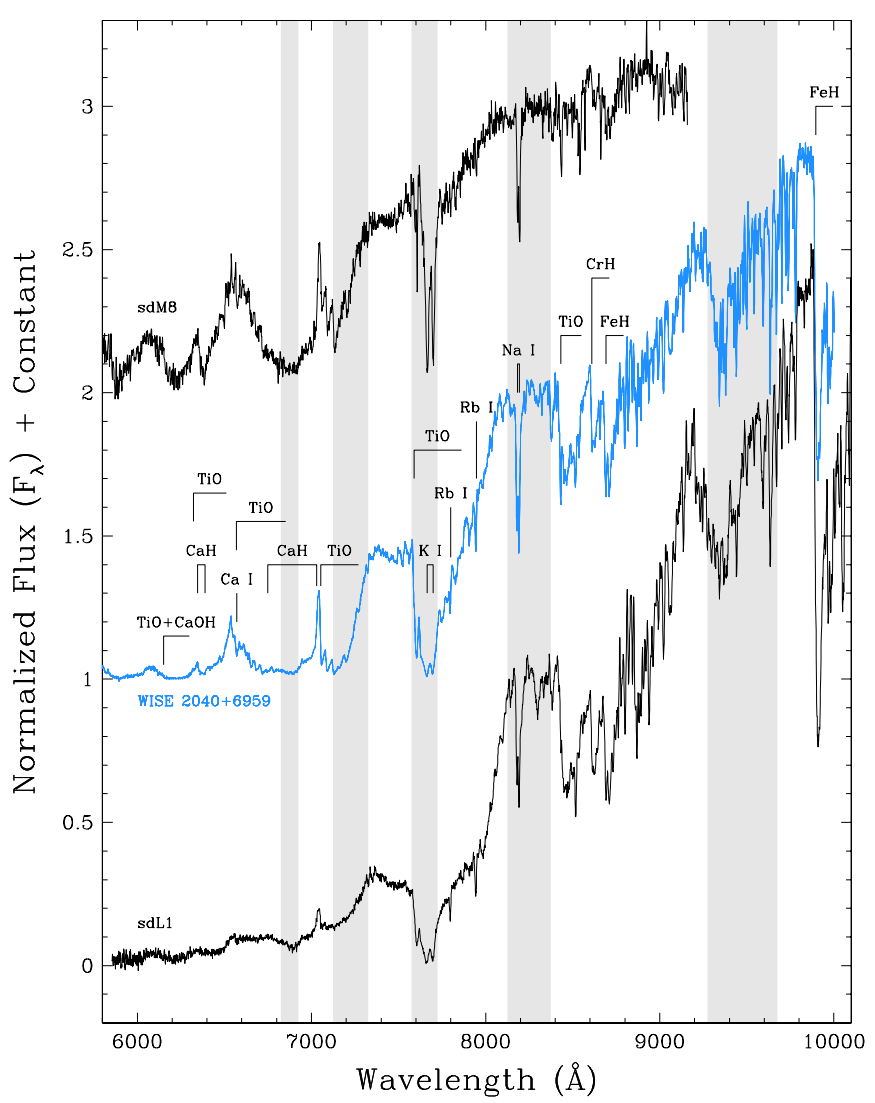

Figure 24. Optical spectra of the sdM8 LSPM J1425+7102 (Lépine et al. 2007) and the sdL1 2MASS 17561080+2815238 (Kirkpatrick et al. 2010) compared to the optical spectrum of WISEA J204027.30+695924.1. Prominent spectral features are marked. Spectra have been normalized at $8250 \AA$ and a constant offset added to the flux to separate the spectra vertically. Regions of telluric absorption are marked by the light gray bands.

(A color version of this figure is available in the online journal.)

infrared spectra of an sdM9.5 and the optically classified sdL1 from Figure 24. These spectra have a similar spectral morphology—notably, strong FeH at $9896 \AA$ and suppressed fluxes at $H$ and $K$ bands relative to $J$. This morphology is similar to that of the sdM9.5 and the sdL1. Two of the AllWISE objects, WISEA J030601.66-033059.0 and WISEA J043535.82+211508.9, also have optical spectra, shown in Figure 25, that we classify as sdL0. Thus, we tentatively classify these of these AllWISE sources as sdL0 objects in the near-infrared, whereas we classify WISEA J004326.26+222124.0-because its spectral morphology is more like 2MASS J17561080+2815238-as an sdL1.

One final L subdwarf discovery from AllWISE, WISEA J005757.64+201304.0, falls at late-L. Figure 28 compares the optical and near-infrared spectra of this object with the L7 standard and with a known sdL7 from the literature. In the optical, WISEA J005757.64+201304.0 fits the overall spectral shape of the normal L7 well except in the strength of the $\mathrm{TiO}$ band at $8432 \AA$, the $\mathrm{CrH}$ band at $8611 \AA$, and the $\mathrm{FeH}$ band at $8692 \AA$, all of which are stronger in the AllWISE object than in the standard. The published sdL7 spectrum, while fitting the overall shape as well as the normal L7 standard, also nicely matches the strengths of the these three discrepant bands. In the near-infrared, the spectrum of the AllWISE object best matches the L7 standard in the $J$ band-although the match is rather poor-but the standard is much redder in the $H$ and $K$ portions of the spectrum. The near-infrared spectrum of the sdL7, on the other hand, provides a better match at $J$ band, an excellent match 


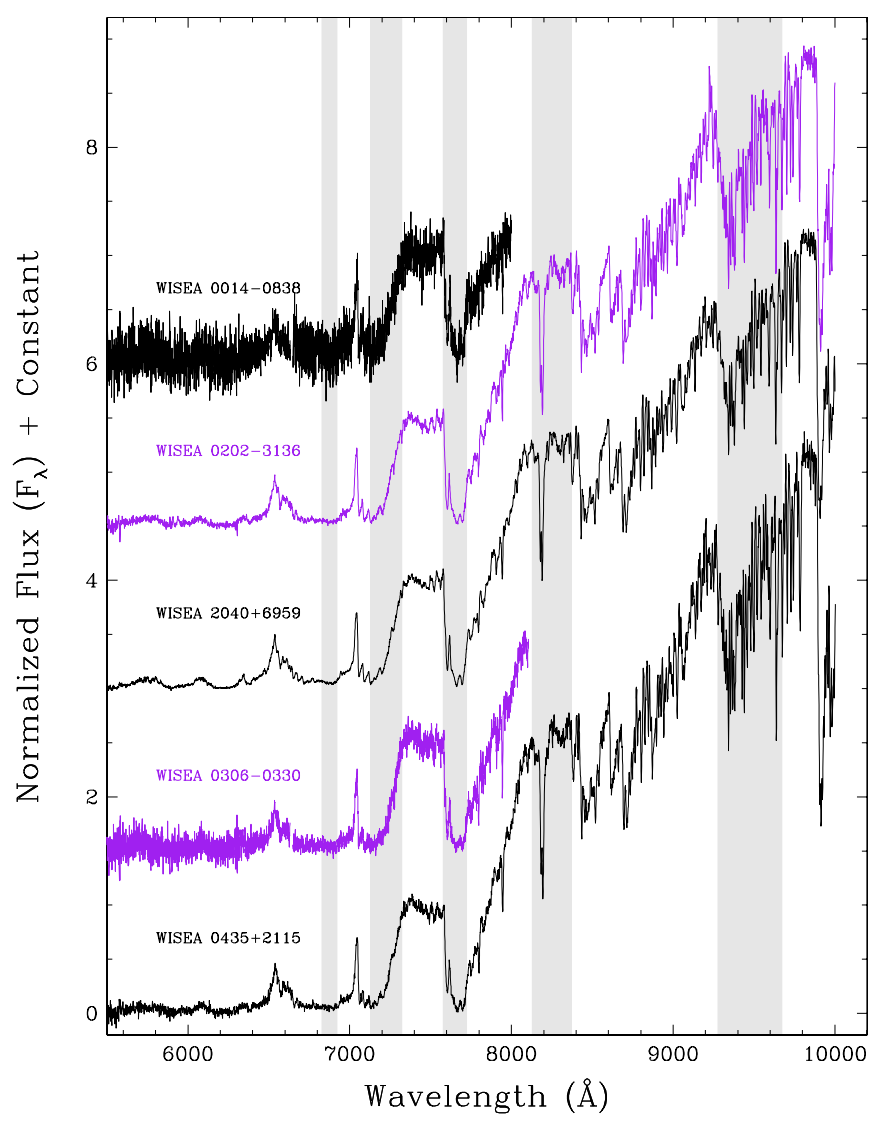

Figure 25. Optical spectra of five early-L subdwarf discoveries from AllWISE: WISEA J001450.17-083823.4, WISEA J020201.25-313645.2 WISEA J204027.30+695924.1, WISEA J030601.66-033059.0, and WISEA J043535.82+211508.9. Spectra have been normalized at $7500 \AA$ and a constant offset added to the flux to separate the spectra vertically. Regions of telluric absorption are marked by the light gray bands.

(A color version of this figure is available in the online journal.)

at $H$, and a much improved match at $K$. For these reasons, we tentatively classify this object as an sdL 7 in both the optical and the near-infrared.

Our follow-up from AllWISE has already added several new $\mathrm{L}$ subdwarfs to the number published to date. Table 6 compiles all of the late-M and $\mathrm{L}$ subdwarfs currently recognized. Of these, 20 were known prior to AllWISE follow-up and eight are from this paper. Additional follow-up of other AllWISE motion sources is certain to reveal others. It is curious that the AllWISE subdwarf discoveries tend to cluster around a type of sdL0. Is this providing a hint regarding the number density of these objects in the solar neighborhood? Table 6 lists the AllWISE motions for all of the known late-M and L subdwarfs detected by WISE. Although a few of the detected objects do not have significant AllWISE motions, most could be easily selected (and many of these were noted as having been found during Quality Assurance reviews). While AllWISE is capable of uncovering subdwarfs throughout the L subdwarf range, objects at mid-L types seem to be lacking. Of the 28 objects in this table, only five fall in the broad range from sdL2 to sdL6. Seventeen objects alone are known within sdM9-sdL1 and another five are known within the sdL7-sdL8 range. (The "schizophrenic" object LSR J1610-0040 is not included in this list since its spectrum defies classification.)

Figure 29 is a rehash of Figure 21 with all known spectroscopically identified late-M and L-type subdwarfs color

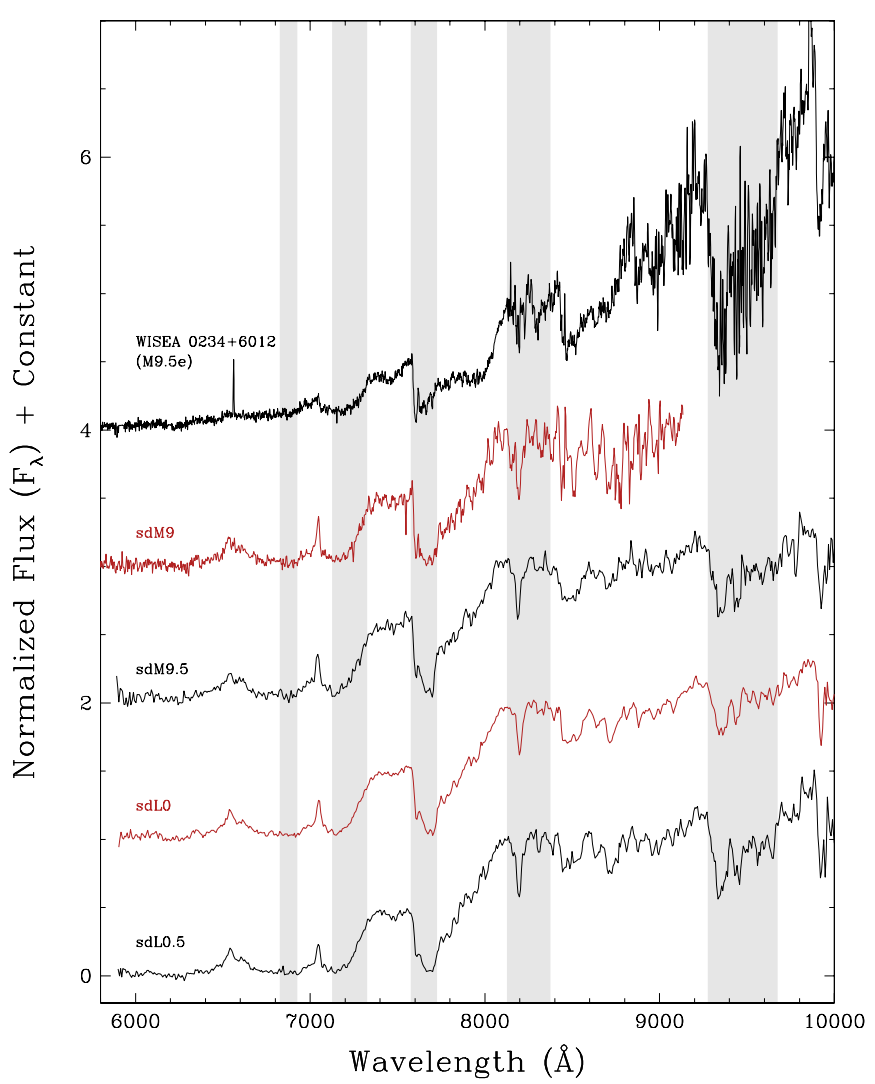

Figure 26. Optical spectrum of a normal M9.5e dwarf (the motion star WISEA J023421.83+601227.3) compared to optical spectra of very late-M and earlyL subdwarfs from the literature: the sdM9 2MASS J16403197+1231068 from Gizis \& Harvin (2006), the sdM9.5 ULAS J115826.62+044746.8 from Lodieu et al. (2010), and the sdL0 ULAS J033350.84+001406.1 and the sdL0.5 ULAS J124425.90+102441.9 from Lodieu et al. (2012). Spectra have been normalized at $7500 \AA$ and a constant offset added to the flux to separate the spectra vertically. Regions of telluric absorption are marked by the light gray bands.

(A color version of this figure is available in the online journal.)

coded. This diagram hints at the possibility that a lightly populated gap may exist between the early-L subdwarf and late- $\mathrm{L}$ subdwarf populations. Such a gap would be expected on theoretical grounds: Lower metallicities generally correspond to older ages. At these older ages, substellar objects have had enough time to cool that a gap in temperature will have opened up between the hottest members of their population and the coldest, lowest mass members of similarly aged hydrogen-burning stars. Such a temperature gap would be wider the older the population, and hence, the lower the metallicity. Figure 29 shows the first signs of what may be a sparsely populated locus of color space corresponding to this temperature gap.

The late-L subdwarf at $(1.92,0.26)$ on Figure 29 is the (e?)sdL7 2MASS J05325346+8246465 (Burgasser et al. 2003). That object has a trigonometric parallax measurement; after comparison to theoretical evolutionary models, Burgasser et al. (2008) find that the resulting luminosity is consistent with the object being a very high-mass brown dwarf just below the hydrogen-burning limit. This would place the object on the brown dwarf side of the $\mathrm{L}$ subdwarf gap, as shown in the figure. The early-L subdwarf at $(1.30,-0.03)$ is the sdL4 2MASS J16262034+3925190; Burgasser (2004) finds that this object is near or below the hydrogen-burning minimum mass limit, consistent with our placement of it on the opposite side of the sparsely populated "subdwarf gap." 


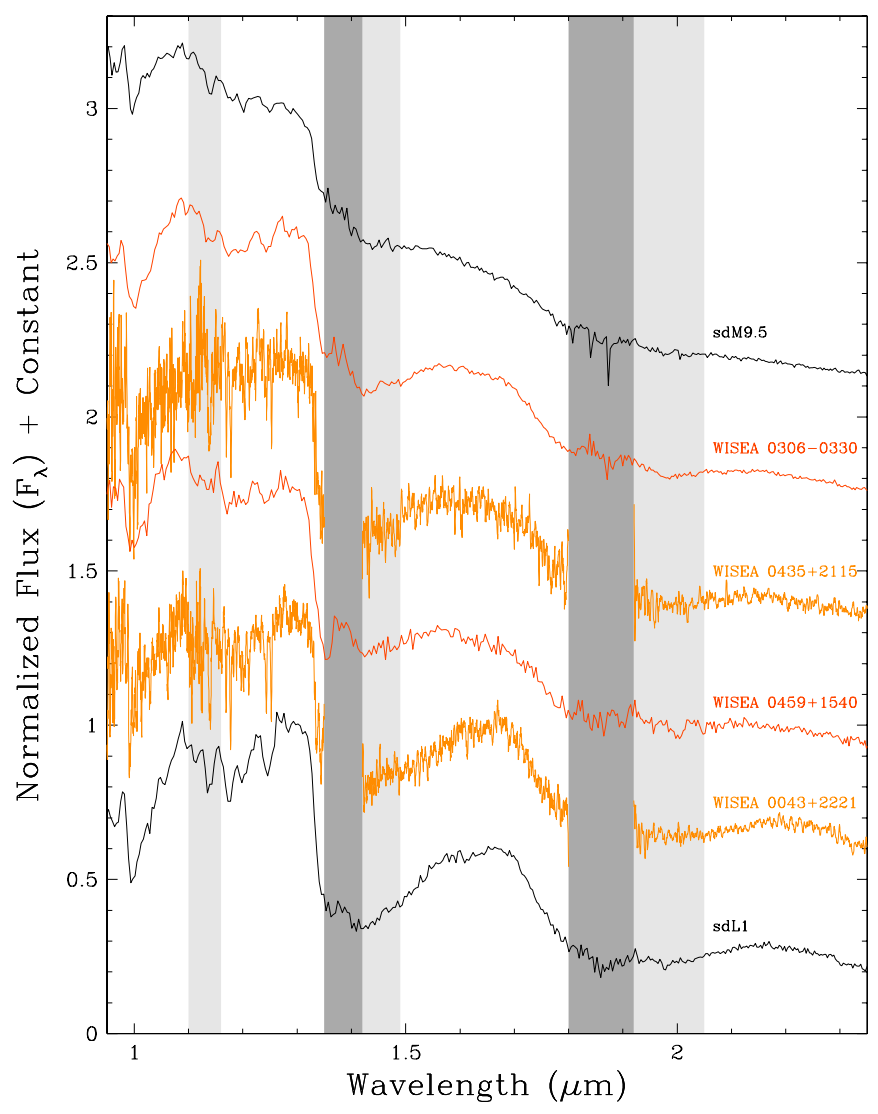

Figure 27. Near-infrared spectra of the sdM9.5 J1013-1356 (Burgasser et al. 2007) and the sdL1 2MASS J17561080+2815238 (Kirkpatrick et al. 2010) compared to the near-infrared spectra of four discoveries from AllWISE: WISEA J030601.66-033059.0, WISEA J043535.82+211508.9, WISEA J045921.21+154059.2 and WISEA J004326.26+222124.0. Regions of telluric absorption are marked by the dark gray and light gray bands as explained in the caption to Figure 23. Spectra have been normalized at $1.28 \mu \mathrm{m}$ and a constant offset added to the flux to separate the spectra vertically.

(A color version of this figure is available in the online journal.)

Despite these checks against limited published results, the zone of L subdwarf avoidance shown on Figure 29 should be regarded only as a cartoon depiction of expectations. With continued follow-up of motion discoveries from AllWISE, it is hoped that researchers will be able to more fully explore the boundaries of the gap, if it is real.

\section{TWO NEW, NEARBY M DWARF SYSTEMS}

There is a single object, WISEA J154045.67-510139.3, occupying the upper right quadrant of Figure 17. This object is very bright, $J=8.96 \pm 0.02 \mathrm{mag}$ and $W 2=7.47 \pm 0.02 \mathrm{mag}$, and has a very large motion, $\mu=2006 \pm 12$ mas yr $^{-1}$, which is the second-highest motion of any of our discoveries. A finder chart is shown in the upper panel of Figure 30. Our optical spectrum (Figure 31) shows this object to be a normal M6 dwarf. The absolute $J$-band magnitude for a typical M6 dwarf is 10.12 mag (Equation (6) of Cruz et al. 2003), which would place this object at a distance of $\sim 5.9 \mathrm{pc}$ if single or $\sim 8.3 \mathrm{pc}$ if an equal-magnitude double. We have gathered positional information on this source from a variety of online archives, as listed in Table 7. Using our fitting code, which is explained in Section 5.2 of Kirkpatrick et al. (2011), we obtain the following astrometric solution with $\chi^{2}=9.5$ and five degrees of freedom: $\mu_{\alpha}=1^{\prime \prime} .951 \pm 0.006 \mathrm{yr}^{-1}, \mu_{\delta}=-0^{\prime \prime} 332 \pm 0^{\prime \prime} 006 \mathrm{yr}^{-1}$, and
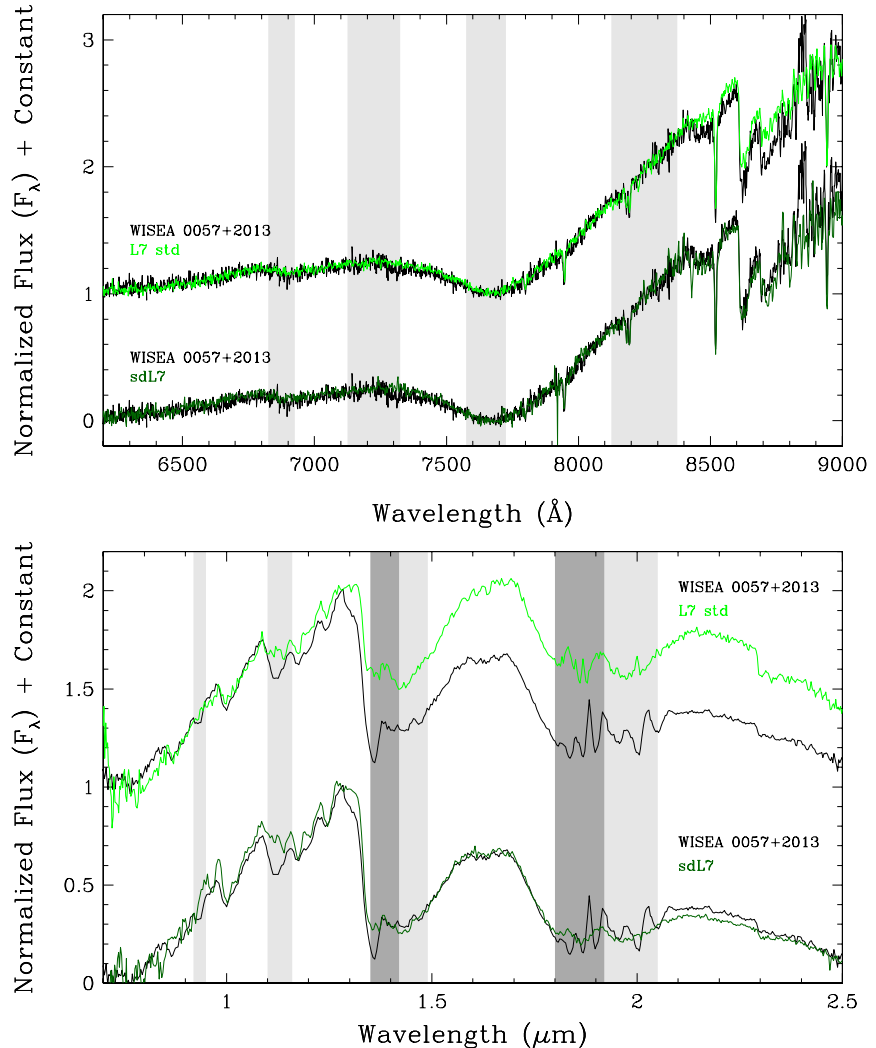

Figure 28. Spectra of WISEA J005757.64+201304.0 compared to the L7 standard and an sdL7 from the literature. (Upper panel) The optical spectrum of the AllWISE object compared to the optical L7 standard DENIS-P J0205.4-1159 (Kirkpatrick et al. 1999) and the sdL7 2MASS J11582077+0435014 (Kirkpatrick et al. 2010). (Lower panel) The near-infrared spectrum of the AllWISE object compared to the near-infrared L7 standard 2MASS J01033203+1935361 and the sdL7 2MASS J11582077+0435014 (both from Kirkpatrick et al. 2010). Normalizations, offsets, and telluric-zone shading are the same as in previous figures.

(A color version of this figure is available in the online journal.)

$\pi=0^{\prime} 165 \pm 0^{\prime} 041(d \approx 6 \mathrm{pc})$. Although the value of the parallax is very fragile given the size of its uncertainty, the available astrometry strongly suggests that this object is close to the Sun, in agreement with our spectrophotometric distance estimate.

A second, bright object is seen next to WISEA J154045.67-510139.3 on Figure 18. This object, WISEA J162702.28-694411.8, has magnitudes and colors of $J=$ $9.16 \pm 0.04 \mathrm{mag}, W 2=7.65 \pm 0.02 \mathrm{mag}, J-W 2=1.52 \pm$ $0.05 \mathrm{mag}$, and $J-K_{s}=0.97 \pm 0.05 \mathrm{mag}$. A finder chart is shown in the lower panel of Figure 30. Our follow-up imaging shows it to be a near equal-magnitude binary separated by 2 '.0. magnitude differences between the two components are found to be $\Delta V=0.18 \pm 0.01 \mathrm{mag}$ and $\Delta I=0.13 \pm$ 0.01 mag. Optical spectra of the two components (Figure 31) show that both are normal M4 dwarfs. The absolute $J$-band magnitude for a typical M4 dwarf is 7.89 mag (Equation (6) of Cruz et al. 2003), which would place this near-equal-magnitude binary at a distance of $\sim 25$ pc. Using additional positional information listed in Table 7, we obtain the following astrometric solution with $\chi^{2}=1.22$ and five degrees of freedom: $\mu_{\alpha}=00^{\prime} 096 \pm 0.007 \mathrm{yr}^{-1}, \mu_{\delta}=00^{\prime \prime} 133 \pm 0.006 \mathrm{yr}^{-1}$, and $\pi=0{ }^{\prime} .008 \pm 0.039$. At face value, a nearby distance seems not to be supported by these data, but we note that the first four (of five) data points were all taken in March or April-i.e., 
Table 6

A List of Known Late-M ( $\geqslant$ M9) and L Subdwarfs

\begin{tabular}{|c|c|c|c|c|c|c|c|c|c|c|}
\hline Designation & $\begin{array}{c}\text { Opt. } \\
\text { Sp. Type } \\
\text { (2) }\end{array}$ & $\begin{array}{c}\text { NIR } \\
\text { Sp. Type }\end{array}$ & Ref. & $\begin{array}{c}\text { 2MASS } J \\
\text { (mag) } \\
(5)\end{array}$ & $\begin{array}{l}\text { 2MASS } H \\
\text { (mag) } \\
(6)\end{array}$ & $\begin{array}{c}\text { 2MASS } K_{s} \\
(\mathrm{mag}) \\
(7)\end{array}$ & $\begin{array}{c}\begin{array}{c}W 1 \\
(\mathrm{mag})\end{array} \\
(8)\end{array}$ & $\begin{array}{c}\begin{array}{c}W 2 \\
(\mathrm{mag})\end{array} \\
(9)\end{array}$ & $\begin{array}{c}\text { AllWISE } \\
\text { R.A. Motion } \\
\left(\mathrm{mas} \mathrm{yr}^{-1}\right) \\
(10)\end{array}$ & $\begin{array}{c}\text { AllWISE } \\
\text { Decl. Motion } \\
\left(\mathrm{mas} \mathrm{yr}^{-1}\right) \\
(11)\end{array}$ \\
\hline WISEA J001450.17-083823.4 & sdL0 & . & 1 & $14.469 \pm 0.026$ & $13.950 \pm 0.026$ & $13.769 \pm 0.044$ & $13.429 \pm 0.025$ & $13.204 \pm 0.030$ & $1392 \pm 92$ & $-406 \pm 93$ \\
\hline 2MASS J00412179+3547133 & $\ldots$ & sdL? & 19 & $15.935 \pm 0.081$ & $15.728 \pm 0.152$ & $15.166 \pm 0.121$ & $14.743 \pm 0.032$ & $14.454 \pm 0.049$ & $13 \pm 133$ & $54 \pm 136$ \\
\hline a & $\ldots$ & sdM9 & 20 & $\ldots$ & $\ldots$ & $\ldots$ & $\ldots$ & $\ldots$ & $\ldots$ & $\ldots$ \\
\hline WISEA J004326.26+222124.0 & $\ldots$ & sdL1 & 1 & $14.871 \pm 0.038$ & $14.226 \pm 0.039$ & $13.931 \pm 0.063$ & $13.688 \pm 0.025$ & $13.373 \pm 0.031$ & $270 \pm 59$ & $-381 \pm 60$ \\
\hline WISEA J005757.65+201304.0 & sdL7 & sdL7 & 1 & $16.317 \pm 0.095$ & $15.449 \pm 0.088$ & $14.974 \pm 0.134$ & $14.317 \pm 0.029$ & $13.872 \pm 0.039$ & $853 \pm 98$ & $-260 \pm 101$ \\
\hline WISEA J020201.25-313645.2 & sdL0 & $\ldots$ & 1 & $15.335 \pm 0.050$ & $14.937 \pm 0.098$ & $14.702 \pm 0.108$ & $14.310 \pm 0.028$ & $13.959 \pm 0.038$ & $-278 \pm 132$ & $-1666 \pm 134$ \\
\hline WISEA J030601.66-033059.0 & sdL0 & sdL0 & 1 & $14.441 \pm 0.026$ & $14.060 \pm 0.040$ & $13.978 \pm 0.048$ & $13.429 \pm 0.025$ & $13.181 \pm 0.027$ & $388 \pm 44$ & $-1258 \pm 47$ \\
\hline ULAS J033350.84+001406.1 & sdL0 & $\ldots$ & 2 & $16.018 \pm 0.111$ & $15.698 \pm 0.178$ & $>16.630$ & $15.077 \pm 0.038$ & $14.765 \pm 0.071$ & $663 \pm 284$ & $-70 \pm 300$ \\
\hline WISEA J043535.82+211508.9 & sdL0 & sdL0 & 1 & $15.011 \pm 0.031$ & $14.682 \pm 0.053$ & $14.594 \pm 0.081$ & $13.999 \pm 0.029$ & $13.668 \pm 0.039$ & $1022 \pm 127$ & $-771 \pm 138$ \\
\hline WISEA J045921.22+154059.2 & $\ldots$ & sdL0 & 1 & $14.957 \pm 0.031$ & $14.613 \pm 0.047$ & $14.300 \pm 0.062$ & $14.093 \pm 0.028$ & $13.851 \pm 0.042$ & $707 \pm 130$ & $-553 \pm 142$ \\
\hline 2MASS J05325346+8246465 & late sdL & late sdL & 17 & $15.179 \pm 0.058$ & $14.904 \pm 0.091$ & $14.918 \pm 0.145$ & $13.824 \pm 0.025$ & $13.260 \pm 0.028$ & $2416 \pm 77$ & $-1450 \pm 83$ \\
\hline$\cdots$ & (e?)sdL7 & (e?)sdL7 & 4 & $\cdots$ & $\cdots$ & $\cdots$ & $\cdots$ & $\cdots$ & $\cdots$ & $\cdots$ \\
\hline$\cdots$ & (e)sdL7: $\alpha$ & (e)sdL7: $\alpha$ & 18 & $\ldots$ & $\ldots$ & $\cdots$ & $\cdots$ & $\cdots$ & $\cdots$ & $\cdots$ \\
\hline$\cdots$ & sdL7 & $\ldots$ & 12 & $\ldots$ & $\cdots$ & $\cdots$ & $\ldots$ & $\cdots$ & $\ldots$ & $\ldots$ \\
\hline$\ldots$ & esdL7 & esdL7 & 24 & $\ldots$ & $\ldots$ & $\ldots$ & $\ldots$ & $\ldots$ & $\ldots$ & $\ldots$ \\
\hline 2MASS J06164006-6407194 & sdL5 & sdL5 & 9 & $16.403 \pm 0.112$ & $16.275 \pm 0.228$ & $>16.381$ & $15.646 \pm 0.030$ & $15.183 \pm 0.042$ & $1438 \pm 162$ & $-63 \pm 177$ \\
\hline 2MASS J06453153-6646120 & sdL8 & sdL8 & 4 & $15.602 \pm 0.067$ & $14.696 \pm 0.070$ & $14.372 \pm 0.084$ & $13.761 \pm 0.023$ & $13.308 \pm 0.023$ & $-851 \pm 45$ & $1134 \pm 44$ \\
\hline SSSPM J1013-1356 & sdM9.5 & sdM9.5 & 12 & $14.621 \pm 0.029$ & $14.382 \pm 0.048$ & $14.398 \pm 0.077$ & $13.797 \pm 0.026$ & $13.604 \pm 0.032$ & $90 \pm 93$ & $-1087 \pm 100$ \\
\hline & sdM9.5 & & 21 & & & & & & $\cdots$ & \\
\hline 2MASS J11582077+0435014 & sdL7 & sdL7 & 4 & $15.611 \pm 0.054$ & $14.684 \pm 0.064$ & $14.439 \pm 0.063$ & $13.695 \pm 0.026$ & $13.361 \pm 0.033$ & $871 \pm 103$ & $-633 \pm 109$ \\
\hline ULAS J115826.62+044746.8 & sdM9.5 & $\ldots$ & 2 & $16.536 \pm 0.130$ & $16.079 \pm 0.201$ & $15.725 \pm 0.186$ & $15.655 \pm 0.051$ & $15.408 \pm 0.121$ & $127 \pm 469$ & $-913 \pm 525$ \\
\hline ULAS J124425.90+102441.9 & sdL0.5 & $\ldots$ & 2 & $16.198 \pm 0.128$ & $15.650 \pm 0.160$ & $>15.400$ & $15.453 \pm 0.041$ & $15.138 \pm 0.088$ & $-84 \pm 310$ & $-174 \pm 337$ \\
\hline$\ldots$ & sdL2? & $\ldots$ & 25 & $\ldots$ & $\ldots$ & $\ldots$ & $\ldots$ & $\ldots$ & $\ldots$ & $\ldots$ \\
\hline SDSS J125637.13-022452.4 & sdL4? & $\cdots$ & 8 & $16.099 \pm 0.105$ & $15.792 \pm 0.148$ & $>15.439$ & $15.210 \pm 0.036$ & $15.005 \pm 0.080$ & $-604 \pm 183$ & $-50 \pm 195$ \\
\hline$\cdots$ & sdL3.5 & sdL3.5 & 10 & $\ldots$ & $\cdots$ & $\cdots$ & $\cdots$ & $\ldots$ & $\cdots$ & $\cdots$ \\
\hline .. & $\cdots$ & esdL3.5 & 24 & $\cdots$ & $\cdots$ & $\cdots$ & $\cdots$ & $\ldots$ & $\ldots$ & $\cdots$ \\
\hline HD $114762 B^{a}$ & - & $\mathrm{d} / \mathrm{sdM} 9$ & 11 & $\ldots$ & $\cdots$ & $\cdots$ & $\cdots$ & $\cdots$ & $\ldots$ & $\cdots$ \\
\hline SDSS J133348.24+273508.8 & sdL3 & $\cdots$ & 25 & $\ldots$ & $\cdots$ & $\cdots$ & $\cdots$ & $\cdots$ & $\cdots$ & $\cdots$ \\
\hline ULAS J135058.86+081506.8 & $\operatorname{sdL} 5 \pm 1$ & esdL4 & 3 & $\ldots$ & $\ldots$ & $\ldots$ & $\ldots$ & $\ldots$ & $\ldots$ & $\ldots$ \\
\hline SDSS J141624.08+134826.7 & sdL7 & sdL7 & 4 & $13.148 \pm 0.021$ & $12.456 \pm 0.027$ & $12.114 \pm 0.021$ & $11.363 \pm 0.022$ & $11.026 \pm 0.020$ & $5 \pm 27$ & $80 \pm 28$ \\
\hline$\cdots$ & d/sdL7 & 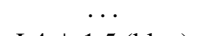 & 5 & $\cdots$ & $\cdots$ & $\cdots$ & $\cdots$ & $\cdots$ & $\cdots$ & $\cdots$ \\
\hline$\cdots$ & L5 & $\mathrm{L} 4 \pm 1.5$ (blue) & 6 & $\cdots$ & $\cdots$ & $\cdots$ & $\cdots$ & $\cdots$ & $\cdots$ & $\cdots$ \\
\hline$\cdots$ & $\mathrm{L} 6 \pm 0.5$ & L6 \pm pec & 7 & $\cdots$ & $\cdots$ & $\cdots$ & $\cdots$ & $\cdots$ & $\cdots$ & $\ldots$ \\
\hline$\cdots$ & $\mathrm{d} / \mathrm{sdL} 7$ & $\mathrm{~d} / \mathrm{sdL} 7$ & 24 & $\cdots$ & $\cdots$ & $\cdots$ & $\cdots$ & $\cdots$ & $\cdots$ & $\cdots$ \\
\hline
\end{tabular}


WISEA J204027.30+695924.1

$\begin{array}{cc}\text { Opt. } & \text { NIR } \\ \text { Sp. Type } & \text { Sp. Type }\end{array}$

Ref.

2MASS $J$
$(\mathrm{mag})$

Designation

(2)

(3)

2MASS J14343616+2202463

SSSPM J1444-2019

$\ldots$
$\mathrm{d} / \mathrm{sdM} 9$

LSR J1610-0040

$\cdots$

2MASS J16262034+3925190

$\cdots$

2MASS J16403197+1231068

sdM9 or sdL

$\mathrm{sdL}$

sd?M6 pec

$\cdots$

d $\cdots$

sdL4

$$
\text { sdL4 }
$$

d/sdM9

dM9/sdL?

sdL1

sdL0

dM8?

1 pec (blue)

Notes. ${ }^{a}$ Also known as 2MASS J13121982+1731016B.

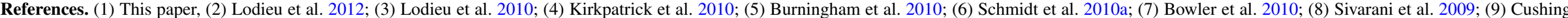

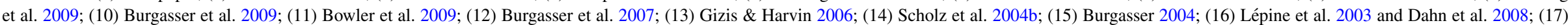

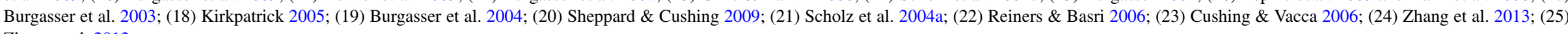
Zhang et al. 2012. 


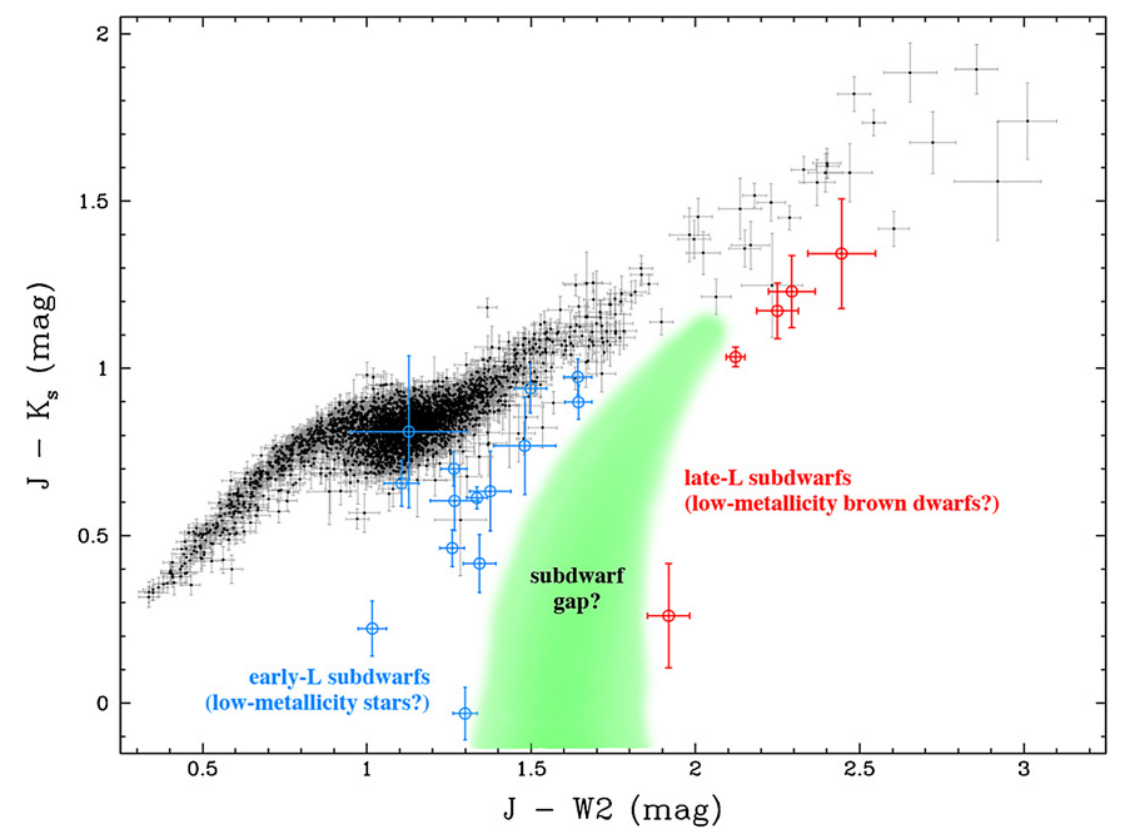

Figure 29. AllWISE motion stars (solid black dots) from Figure 21, with the three possible $\mathrm{T}$ dwarfs omitted for clarity. The locations of known late-M and $\mathrm{L}$ subdwarfs from Table 6 are shown by open circles. Those with spectral types earlier than sdL5 are shown in blue and those with types later than sdL5 are shown in red. The wedge (green zone) depicts an area of color space where L subdwarfs may rarely be found. See the text for details.

(A color version of this figure is available in the online journal.)

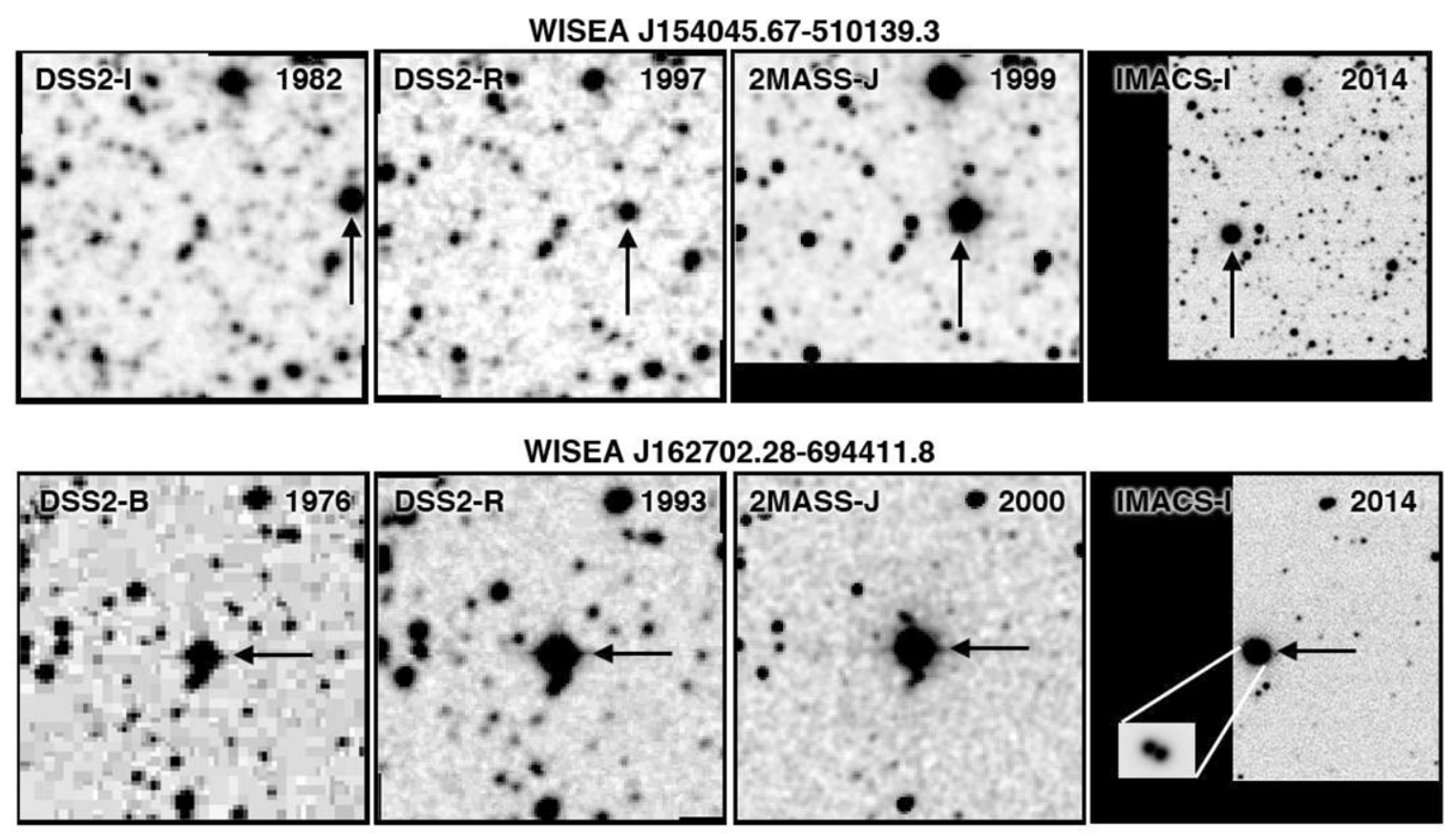

Figure 30. Images from DSS2, 2 MASS, and Magellan/IMACS for two nearby M dwarf systems. (Upper panel) DSS2-I, DSS2- $R$, 2MASS- $J$, and IMACS- $I$ images for the motion object WISEA J154045.67-510139.3. (Lower panel) DSS2- $B$, DSS2- $R$, 2MASS- $J$, and IMACS-I images for the motion object WISEA $\mathrm{J} 162702.28-694411.8$. In both panels, the epoch of each image is labeled and the position of the motion star in indicated by an arrow. Each subpanel is $2^{\prime}$ square with north up and east to the left. The inset on the IMACS-I image for WISEA J162702.28-694411.8AB shows the individual components of the system.

at nearly the same parallax factor. Only the final observation, taken in September, provides any leverage regarding the size of the parallax. Moreover, the binarity of the source may be complicating clean measurements of the photocenter. Additional astrometric monitoring is needed.

WISEA J154045.67-510139.3 and WISEA J162702.28694411.8 have Galactic coordinates of $(328.0,+3.4)$ and $(319.7,-14.2)$, respectively, and fall in the lower right quadrant of Figure 15 (middle panel). One of the objects in the Luhman (2014) list, WISE J163348.95-680851.6 $(J=11.19 \mathrm{mag}$, $J-K_{s}=1.12 \mathrm{mag}, J-W 2=2.20 \mathrm{mag}$ ), is located at Galactic coordinates of $(321: 0,-13.4)$, and may be another close object, likely a late-M or early-L dwarf. The density of WISE motion discoveries in this quadrant demonstrates that a large area around the Galactic Center has been poorly surveyed by earlier searches for nearby stars. 
Table 7

Additional Astrometric Data for The Two Nearby M-Dwarf Systems

\begin{tabular}{lcccccc}
\hline \hline MJD & $\begin{array}{c}\text { Calendar } \\
\text { Date } \\
(2)\end{array}$ & $\begin{array}{c}\text { J2000 R.A. } \\
(\mathrm{deg}) \\
(3)\end{array}$ & $\begin{array}{c}\text { J2000 Decl. } \\
(\mathrm{deg}) \\
(1)\end{array}$ & $\begin{array}{c}\text { R.A. Error } \\
\left({ }^{\prime \prime}\right) \\
(5)\end{array}$ & $\begin{array}{c}\text { Decl. Error } \\
\left({ }^{\prime \prime}\right) \\
(6)\end{array}$ & Notes \\
\hline \multicolumn{7}{c}{ WISEA J154045.67-510139.3 } \\
\hline 45078.690 & 1982 Apr 19 & 235.166113 & -51.025275 & 0.333 & 0.333 & DSS2 I-band \\
50554.606 & 1997 Apr 16 & 235.179017 & -51.026369 & 0.333 & 0.333 & DSS2 R-band \\
51364.022 & 1999 Jul 05 & 235.180914 & -51.026588 & 0.06 & 0.06 & 2MASS \\
55251.096 & 2010 Feb 24 & 235.190210 & -51.027578 & 0.071 & 0.067 & WISE All-Sky \\
55432.482 & 2010 Aug 24 & 235.190481 & -51.027619 & 0.044 & 0.041 & WISE three-band Cryo \\
\hline \multicolumn{7}{c}{ WISEA J162702.28-694411.8 } \\
\hline 42869.726 & 1976 Apr 01 & 246.75713 & -69.73792 & 0.5 & 0.5 & DSS2 B-band \\
48352.721 & 1991 Apr 06 & 246.75809 & -69.737244 & 0.33 & 0.33 & DSS2 I-band \\
51652.288 & 2000 Apr 18 & 246.758802 & -69.736984 & 0.06 & 0.06 & 2MASS \\
55264.727 & 2010 Mar 09 & 246.759567 & -69.736625 & 0.066 & 0.064 & WISE All-Sky \\
55444.838 & 2010 Sep 05 & 246.759596 & -69.736595 & 0.041 & 0.039 & WISE three-band Cryo
\end{tabular}

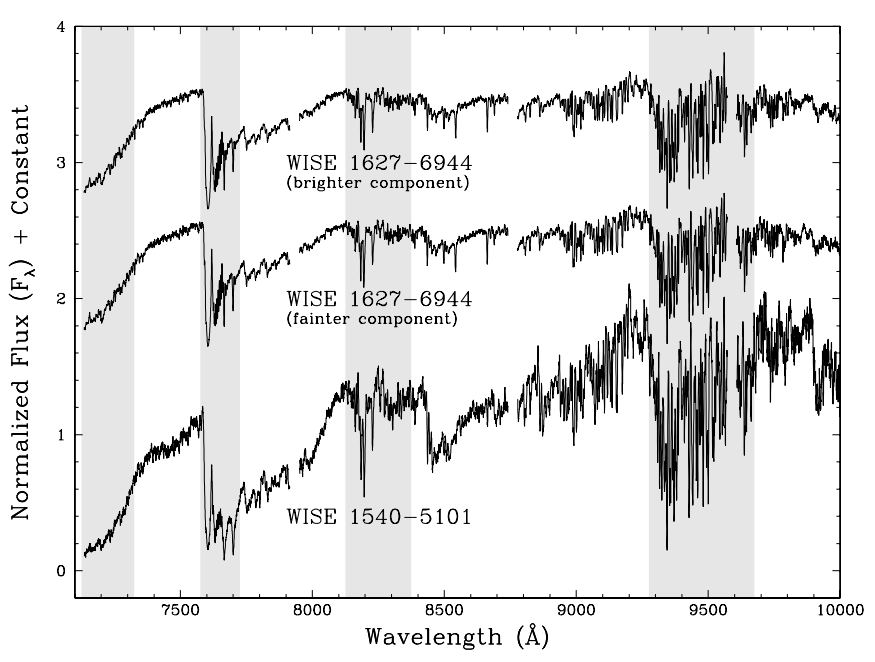

Figure 31. Optical spectra of the two components of the WISEA J162702.28-694411.8AB system (top spectra) and WISEA J154045.67-510139.3 (bottom spectrum). Spectra have been normalized at $7500 \AA$ and a constant offset added to the flux to separate the spectra vertically. Regions of telluric absorption are marked by the light gray bands.

\section{CONCLUSIONS}

We have characterized the motion measurements contained within the AllWISE Data Release. We have presented a list of 3525 motion objects lacking previous literature in SIMBAD and find two very interesting results. The first is that AllWISE is revealing many more examples of low-metallicity objects near the stellar/substellar break, allowing researchers to probe the region near the hydrogen-burning minimum mass for objects formed early in the history of the Milky Way. The second is that AllWISE is continuing the WISE legacy of uncovering previously overlooked, very nearby objects. Prior to AllWISE processing, WISE had already revealed several nearby systems never before recognized -e.g., the L+T binary WISE J104915.57-531906.1 at $2.0 \mathrm{pc}$ (Luhman 2013) and the T6 dwarf WISEPC J150649.97+702736.0 (Kirkpatrick et al. 2011) possibly as close as 3.4 pc (Marsh et al. 2013); AllWISE has already contributed another overlooked object-WISEA $\mathrm{J} 154045.67-510139.3(\sim 5.9 \mathrm{pc})$ - to this distinguished list of WISE discoveries within the canonical 8 pc sample. The motion measurements provided by AllWISE will likely provide researchers many new gifts over the years to come.
This publication makes use of data products from WISE, which is a joint project of the University of California, Los Angeles, and the Jet Propulsion Laboratory (JPL)/California Institute of Technology (Caltech), funded by the National Aeronautics and Space Administration (NASA). This research has made use of the NASA/IPAC Infrared Science Archive, which is operated by JPL/Caltech, under contract with NASA. We are indebted to the SIMBAD database and the VizieR catalog access tool, provided by CDS, Strasbourg, France, and we acknowledge use of the Database of Ultracool Parallaxes maintained by Trent Dupuy. We thank our referee, whose critique of the original draft resulted in a clearer, more complete paper. We thank Fiona Harrison, George Djorgovski, Brian Mazur, and Barry Madore for PI-ing telescope time used for spectroscopic follow-up and are grateful for the time allotted by Caltech, NASA/IRTF, and the Carnegie Observatories. We also thank Nicolas Lodieu, John Gizis, and Sebastien Lépine for providing published spectra of subdwarfs.

Facilities: WISE, Hale(Double Spectrograph), Keck:I(LRIS), Keck:II(DEIMOS, NIRSPEC), IRTF(SpeX), Magellan:Baade (IMACS).

\section{REFERENCES}

Ali, B. 2014, AAS Meeting 223, 441.37

Bidelman, W. P. 1985, ApJS, 59, 197

Bowler, B. P., Liu, M. C., \& Cushing, M. C. 2009, ApJ, 706, 1114

Bowler, B. P., Liu, M. C., \& Dupuy, T. J. 2010, ApJ, 710, 45

Burgasser, A. J. 2004, ApJL, 614, L73

Burgasser, A. J., Cruz, K. L., \& Kirkpatrick, J. D. 2007, ApJ, 657, 494

Burgasser, A. J., Kirkpatrick, J. D., Burrows, A., et al. 2003, ApJ, 592, 1186

Burgasser, A. J., McElwain, M. W., Kirkpatrick, J. D., et al. 2004, AJ, 127, 2856

Burgasser, A. J., Vrba, F. J., Lépine, S., et al. 2008, ApJ, 672, 1159

Burgasser, A. J., Witte, S., Helling, C., et al. 2009, ApJ, 697, 148

Burningham, B., Leggett, S. K., Lucas, P. W., et al. 2010, MNRAS, 404, 1952

Castro, P. J., Gizis, J. E., Harris, H. C., et al. 2013, ApJ, 776, 126

Cruz, K. L., Reid, I. N., Kirkpatrick, J. D., et al. 2007, AJ, 133, 439

Cruz, K. L., Reid, I. N., Liebert, J., Kirkpatrick, J. D., \& Lowrance, P. J. 2003, AJ, 126,2421

Cushing, M. C., Looper, D., Burgasser, A. J., et al. 2009, ApJ, 696, 986

Cushing, M. C., \& Vacca, W. D. 2006, AJ, 131, 1797

Cushing, M. C., Vacca, W. D., \& Rayner, J. T. 2004, PASP, 116, 362

Dahn, C. C., Harris, H. C., Levine, S. E., et al. 2008, ApJ, 686, 548

Day-Jones, A. C., Pinfield, D. J., Napiwotzki, R., et al. 2008, MNRAS, 388,838

Dressler, A., Bigelow, B., Hare, T., et al. 2011, PASP, 123, 288

Dupuy, T. J., \& Liu, M. C. 2012, ApJS, 201, 19

Faber, S. M., Phillips, A. C., Kibrick, R. I., et al. 2003, Proc. SPIE, 4841, 1657 
Finch, C. T., Zacharias, N., \& Henry, T. J. 2010, AJ, 140, 844

Gianninas, A., Bergeron, P., \& Ruiz, M. T. 2011, ApJ, 743, 138

Gizis, J. E. 2002, ApJ, 575, 484

Gizis, J. E., \& Harvin, J. 2006, AJ, 132, 2372

Harrington, R. S., \& Dahn, C. C. 1980, AJ, 85, 454

Harrington, R. S., Dahn, C. C., Kallarakal, V. V., et al. 1993, AJ, 105, 1571

Henry, T. J., Jao, W.-C., Subasavage, J. P., et al. 2006, AJ, 132, 2360

Henry, T. J., Kirkpatrick, J. D., \& Simons, D. A. 1994, AJ, 108, 1437

Høg, E., Fabricius, C., Makarov, V. V., et al. 2000, A\&A, 355, L27

Keenan, P. C., \& Keller, G. 1953, ApJ, 117, 241

Keenan, P. C., \& Pitts, R. E. 1980, ApJS, 42, 541

Kerber, F., Mignani, R. P., Smart, R. L., \& Wicenec, A. 2008, A\&A, 479, 155

Kirkpatrick, J. D. 2005, ARA\&A, 43, 195

Kirkpatrick, J. D., Beichman, C. A., \& Skrutskie, M. F. 1997, ApJ, 476, 311

Kirkpatrick, J. D., Cushing, M. C., Gelino, C. R., et al. 2011, ApJS, 197, 19

Kirkpatrick, J. D., Gelino, C. R., Cushing, M. C., et al. 2012, ApJ, 753, 156

Kirkpatrick, J. D., Henry, T. J., \& McCarthy, D. W., Jr. 1991, ApJS, 77, 417

Kirkpatrick, J. D., Looper, D. L., Burgasser, A. J., et al. 2010, ApJS, 190, 100

Kirkpatrick, J. D., McGraw, J. T., Hess, T. R., et al. 1994, ApJS, 94, 749

Kirkpatrick, J. D., Reid, I. N., Liebert, J., et al. 1999, ApJ, 519, 802

Kirkpatrick, J. D., Reid, I. N., Liebert, J., et al. 2000, AJ, 120, 447

Lawson, W. A., Crause, L. A., Mamajek, E. E., \& Feigelson, E. D. 2002, MNRAS, 329, L29

Lépine, S., Rich, R. M., \& Shara, M. M. 2003, ApJL, 591, L49

Lépine, S., Rich, R. M., \& Shara, M. M. 2007, ApJ, 669, 1235

Lépine, S., \& Shara, M. M. 2005, AJ, 129, 1483

Lodieu, N., Espinoza Contreras, M., Zapatero Osorio, M. R., et al. 2012, A\&A, 542, A 105

Lodieu, N., Zapatero Osorio, M. R., Martín, E. L., Solano, E., \& Aberasturi, M. 2010, ApJL, 708, L107

Luhman, K. L. 2001, ApJ, 560, 287

Luhman, K. L. 2013, ApJL, 767, L1

Luhman, K. L. 2014, ApJ, 781, 4

Luyten, W. J. 1979a, The Luyten Half Second Catalog (2nd ed.; Minneapolis, MN: Univ. of Minnesota)

Luyten, W. J. 1979b, The New Luyten Two Tenths Catalog (Minneapolis, MN: Univ. of Minnesota)

Maheswar, G., Manoj, P., \& Bhatt, H. C. 2003, A\&A, 402, 963

Mainzer, A., Bauer, J., Grav, T., et al. 2011, ApJ, 731, 53

Marsh, K. A., Wright, E. L., Kirkpatrick, J. D., et al. 2013, ApJ, 762, 119

Martín, E. L., Phan-Bao, N., Bessell, M., et al. 2010, A\&A, 517, A5

McCaughrean, M. J., Close, L. M., Scholz, R.-D., et al. 2004, A\&A, 413, 1029

McLean, I. S., Becklin, E. E., Bendiksen, O., et al. 1998, Proc. SPIE, 3354, 566

McLean, I. S., Graham, J. R., Becklin, E. E., et al. 2000, Proc. SPIE, 4008, 1048

McLean, I. S., McGovern, M. R., Burgasser, A. J., et al. 2003, ApJ, 596, 561
Oke, J. B., Cohen, J. G., Carr, M., et al. 1995, PASP, 107, 375

Rajpurohit, A. S., Reylé, C., Allard, F., et al. 2013, A\&A, 556, A15

Rayner, J. T., Cushing, M. C., \& Vacca, W. D. 2009, ApJS, 185, 289

Rayner, J. T., Toomey, D. W., Onaka, P. M., et al. 2003, PASP, 115, 362

Reid, I. N., Cruz, K. L., Kirkpatrick, J. D., et al. 2008, AJ, 136, 1290

Reid, N. 1997, in ASP Conf. Ser. 127, Proper Motions and Galactic Astronomy, ed. R. M. Humphreys (San Francisco, CA: ASP), 63

Reiners, A., \& Basri, G. 2006, AJ, 131, 1806

Riaz, B., Gizis, J. E., \& Harvin, J. 2006, AJ, 132, 866

Robertson, T. H. 1984, AJ, 89, 1229

Roeser, S., \& Bastian, U. 1988, A\&AS, 74, 449

Röser, S., Schilbach, E., Schwan, H., et al. 2008, A\&A, 488, 401

Salim, S., \& Gould, A. 2003, ApJ, 582, 1011

Schilbach, E., Röser, S., \& Scholz, R.-D. 2009, A\&A, 493, L27

Schmidt, S. J., West, A. A., Burgasser, A. J., Bochanski, J. J., \& Hawley, S. L. 2010a, AJ, 139, 1045

Schmidt, S. J., West, A. A., Hawley, S. L., \& Pineda, J. S. 2010b, AJ, 139, 1808

Scholz, R.-D., Lehmann, I., Matute, I., \& Zinnecker, H. 2004a, A\&A, 425, 519

Scholz, R.-D., Lodieu, N., \& McCaughrean, M. J. 2004b, A\&A, 428, L25

Sheppard, S. S., \& Cushing, M. C. 2009, AJ, 137, 304

Simcoe, R. A., Burgasser, A. J., Bernstein, R. A., et al. 2008, Proc. SPIE, 7014, $70140 \mathrm{U}$

Simcoe, R. A., Burgasser, A. J., Bochanski, J. J., et al. 2010, Proc. SPIE, 7735, 773514

Sivarani, T., Lépine, S., Kembhavi, A. K., \& Gupchup, J. 2009, ApJL, 694, L140

Stephenson, C. B. 1986a, AJ, 91, 144

Stephenson, C. B. 1986b, AJ, 92, 139

Teegarden, B. J., Pravdo, S. H., Hicks, M., et al. 2003, ApJL, 589, L51

Vacca, W. D., Cushing, M. C., \& Rayner, J. T. 2003, PASP, 115, 389

van Altena, W. F., Lee, J. T., \& Hoffleit, E. D. 1995, The General Catalogue of Trigonometric [Stellar] Parallaxes (4th ed., New Haven, CT: Yale Univ. Observatory)

van de Kamp, P. 1953, PASP, 65, 73

van Leeuwen, F. 2007, A\&A, 474, 653

Walker, A. R. 1983, SAAOC, 7, 106

West, A. A., Hawley, S. L., Bochanski, J. J., et al. 2008, AJ, 135, 785

Wright, E. L., Eisenhardt, P. R. M., Mainzer, A. K., et al. 2010, AJ, 140, 1868

Wright, E. L., Kirkpatrick, J. D., Gelino, C. R., et al. 2013, AJ, 147, 61

Wroblewski, H., \& Torres, C. 1996, A\&AS, 115, 481

Zacharias, N., Finch, C. T., Girard, T. M., et al. 2013, AJ, 145, 44

Zhang, Z. H., Pinfield, D. J., Burningham, B., et al. 2012, poster presented at "50 Years of Brown Dwarfs: From Theoretical Prediction to Astrophysical Studies": http://www.mpia.de/homes/joergens/ringberg2012_proc/zhang.pdf

Zhang, Z. H., Pinfield, D. J., Burningham, B., et al. 2013, European Physical Journal Web of Conferences, 47, 6007 